\title{
GLOBAL EXISTENCE OF WEAK SOLUTIONS TO THE REGULARIZED HOOKEAN DUMBBELL MODEL *
}

\author{
LINGYUN ZHANG ${ }^{\dagger}$, HUI ZHANG ${ }^{\ddagger}$, AND PINGWEN ZHANG ${ }^{\S}$
}

\begin{abstract}
We consider a regularized Hookean dumbbell model in dilute polymeric solutions. Compared with the classical model, this model here is more natural, in which appear a macro diffusive term $\varepsilon \triangle_{x} \psi$ and Friedrichs mollifiers with a parameter $\alpha$. Based on a compactness argument, the global existence of weak solutions to this model is established in the framework of the Rothe method. By a rigorous limiting process $\varepsilon \rightarrow 0_{+}$, we also obtain the global existence of weak solutions to the reduced model with $\varepsilon=0$.
\end{abstract}

Key words. global existence, Hookean dumbbell model, dilute polymer solutions, Navier-Stokes equation, Fokker-Planck equation, Rothe method

AMS subject classifications. 76D03, 82C31, 82D60

\section{Introduction}

In this paper, we investigate the global existence of weak solutions to a regularized Hookean dumbbell model for dilute polymeric fluids. In dilute polymer solutions, the polymer coils rarely overlap, so the interactions among polymer chains can be neglected. The polymer chains can be modelled by dumbbells, each with two beads connected by a single spring. The configuration of the spring then specifies the conformation of the polymer.

Denoting by $\mathbf{u}$ the velocity and by $p$ the pressure, the governing equations for the incompressible polymeric fluids are

$$
\begin{gathered}
\frac{\partial \mathbf{u}}{\partial t}+\left(\mathbf{u} \cdot \nabla_{\mathbf{x}}\right) \mathbf{u}-\nu \Delta_{\mathbf{x}} \mathbf{u}+\nabla_{\mathbf{x}} p=\nabla_{\mathbf{x}} \cdot \tau \quad \text { in } \Omega \times(0, T], \\
\nabla_{\mathbf{x}} \cdot \mathbf{u}=0 \quad \text { in } \Omega \times(0, T] .
\end{gathered}
$$

Here $\Omega$ is a bounded open set in $\mathbb{R}^{d}, d=2$ or $3, \nu>0$ is the viscosity of the solvent, and $\tau$ is an extra stress tensor, which takes the form

$$
\begin{aligned}
\tau & =k \omega(\mathbf{C}(\psi)-\rho(\psi) \mathbf{I}) \quad \text { in } \Omega \times(0, T], \\
\mathbf{C}(\psi) & =\int_{D}\left(\nabla_{q} U \otimes \mathbf{q}\right) \psi(\mathbf{x}, \mathbf{q}, t) d \mathbf{q} \quad \text { in } \Omega \times(0, T], \\
\rho(\psi) & =\int_{D} \psi(\mathbf{x}, \mathbf{q}, t) d \mathbf{q} \quad \text { in } \Omega \times(0, T] .
\end{aligned}
$$

Here $\kappa, \omega>0$ denote the Boltzmann constant and the absolute temperature, respectively. I is the unit $d \times d$ tensor, $U$ is the spring potential. This stress tensor $\tau$

*Recevied: May 25, 2007; accepted (in revised version): October 29, 2007. Communicated by Claude Le Bris.

${ }^{\dagger}$ LMAM and School of Mathematical Sciences, Peking University, Beijing 100871, P.R. China (lingyun@pku.edu.cn).

${ }^{\ddagger}$ School of Mathematical Sciences, Beijing Normal University, Beijing 100875, P.R. China (hzhang@bnu.edu.cn). This work is supported in part by an Alexander von Humboldt Fellowship at Max-Planck Institute for Mathematical Science in Leipzig, NSFC for Project 10401008 and the key basic research project of the Ministry of Chinese Education 107016.

$\S_{\text {LMAM }}$ and School of Mathematical Sciences, Peking University, Beijing 100871, P.R. China (pzhang@pku.edu.cn). This work is supported in part by the state key basic research project of China 2005CB321704 and NSFC for Distinguished Young Scholars 10225103. 
represents the polymer's contribution to stress and is a functional of $\psi$, the probability density function (pdf) describing the configuration of the polymers, which satisfies a Fokker-Planck equation $[6,7,8]$ :

$$
\frac{\partial \psi}{\partial t}+\left(\mathbf{u} \cdot \nabla_{x}\right) \psi+\nabla_{\mathbf{q}} \cdot\left(\left(\nabla_{\mathbf{x}} \mathbf{u}\right) \mathbf{q} \psi\right)=\frac{1}{2 \lambda} \nabla_{\mathbf{q}} \cdot\left(\nabla_{\mathbf{q}} \psi+\nabla_{\mathbf{q}} U \psi\right) \text { in } \Omega \times D \times(0, T] .
$$

Here $\lambda>0$ is a relaxation time and $D$ is the domain of $\mathbf{q}$. Thus, (1.1)-(1.6) composes a Navier-Stokes-Fokker-Planck system. Certainly the dynamics of $\mathbf{q}$ can also be described by a stochastic differential equation [6,9], which is equivalent to the Fokker-Planck equation.

For the potential form $U$ between two beads, there are two types popularly used. One is called the FENE (finitely extensible nonlinear elastic) potential,

$$
D=B\left(\mathbf{0}, Q_{\max }\right), U(\mathbf{q})=-\frac{Q_{\max }^{2}}{2} \ln \left(1-|\mathbf{q}|^{2} / Q_{\max }^{2}\right),
$$

where $Q_{\max }$ is the maximum dumbbell extension and $B\left(\mathbf{0}, Q_{\max }\right)$ denotes a ball with the center $\mathbf{0}$ and the radius $Q_{\max }$. The other is called the linear Hookean potential,

$$
D=\mathbb{R}^{d}, U(\mathbf{q})=\frac{1}{2}|\mathbf{q}|^{2} .
$$

Although the FENE dumbbell model is more practical from a physical point of view, the Hookean dumbbell model is a very valid approximation when the molecule is stretched to no more than about a third of its maximum extension. It can also predict qualitatively some of the nonlinear rheological behavior of dilute solutions. The other distinguished feature is that the system (1.1)-(1.6) for the Hookean dumbbell model can be rigorously closed by the second moments, which leads to the well-known Oldroyd-B model. Further detailed background information for these models can be found in $[6,7,8,17,25]$.

The mathematical theory of these models has drawn considerable attention from the mathematical community in recent years $[1,2,4,9,10,14,15,18,19,20,21,22$, $27,31]$. A review has been given in [19]. The local existences have been established in $[9,15,18,27,31]$. The global existences have been obtained for some special models or under some special conditions $[4,10,14,20,21,22]$. Jourdain et al. [14] restricted to the simple case of Hookean dumbbells in a shear flow. Lions and Masmoudi [21] considered the Oldroyd type model for general initial conditions. However, they investigated a special case through taking one parameter of the model to be zero. In addition, their method does not seem to extend to more general cases. Recently, Lions and Masmoudi [22] investigated the corotational FENE model. Lin, Liu and Zhang [20] studied a micro-macro model for polymeric fluid provided that the initial data is not far from the equilibrium. Bonito et al. [4] were concerned with a simplified Hookean dumbbell stochastic model and gave the global existence of the solution with small data in Banach spaces. Fernández-Cara et al. [10] concentrated on the Oldroyd type models. They derived both local existence for arbitrary regular data and global existence for small data.

Up to now, it is still an open problem to find global in time solutions to the classical dumbbell model for arbitrary data. However, Barrett and Süli [2] made great progress in this related field. They found some simplifications in the derivation of the classical dumbbell model and presented a regularized dumbbell model which is more natural with respect to the classical dumbbell model. Moreover, they proved the 
global existence of weak solutions to the regularized FENE dumbbell model. Compared with the classical model, this regularized model has two noteworthy features, in which appear a macro diffusive term $\varepsilon \triangle_{x} \psi$ and Friedrichs mollifiers with a parameter $\alpha$. In fact, this diffusive term and Friedrichs mollifiers really appear in the derivation procedure of the model. But in the classical derivation this diffusive term is omitted in virtue of $\varepsilon \ll 1$. Moreover, in the classical derivation, the Friedrichs mollifiers are approximated by identity operators in order to simplify the model and then higher regularity of $\mathbf{u}$ and $\psi$ are required, while Barrett and Süli [2] refrained from performing such approximations. They replaced the Friedrichs mollifiers by their isotropic counterparts since the anisotropic Friedrichs mollifiers need to act in all possible directions q contained in the balanced set $D$. Furthermore, some advantages of this regularized model have been described in Lozinski [23], Lozinski, Owens and Fang [24] and Schieber [29].

As a continuation of the work of Barrett and Süli [2], this paper is devoted to the global existence of the regularized Hookean dumbbell model, which couples NavierStokes equations to nonlinear Fokker-Planck equations. From the technical viewpoint this regularized model exemplifies many analytical difficulties which are encountered in the study of complex models. Furthermore, it is possible to give some hints to the theoretical analysis of the classical model through investigation to this regularized model. We have partly completed this analysis for the regularized Hookean dumbbell model. We firstly obtain the global existence of weak solutions to the regularized Hookean dumbbell model. Then we investigate the reduced Hookean dumbbell model with $\varepsilon=0$ by the limiting process $\varepsilon \rightarrow 0_{+}$. However, we could not pass to the limit $\alpha \rightarrow 0_{+}$, and then we fail to obtain the global existence for the classical Hookean dumbbell model as $(\alpha, \varepsilon) \rightarrow(0,0)$. Certainly, we also expect that the mathematical approach here can be extended to the mathematical analysis of other models.

For this regularized dumbbell model considered, and for the particular technique of the mathematical analysis in this paper, the Hookean model presents an additional difficulty with respect to the FENE model. Although it seems that the Hookean model is simpler than the FENE model, there are different singularities in them. In the FENE model, the potential explodes as $\mathbf{q}$ approaches a finite value, while in the Hookean model, q extends in length unboundedly and the potential is unbounded because of the unboundedness of $D$. Note that it is difficult in the well-posedness analysis to deal with the term $\left(\nabla_{x} \mathbf{u}\right) \mathbf{q} \psi$ in (1.6), which couples $\mathbf{u}$ and $\psi$ and contains q. Barrett and Süli [2] have used the boundedness of $D$ to deal with this term and get a priori estimates for $\psi$. Because of the unboundedness of $D$ in the Hookean model, here we have to choose a different approach to deal with this term.

Our main results of this paper are the global existence of weak solutions to the regularized Hookean dumbbell model (see Theorem 2.1 in Section 2)and to the reduced Hookean dumbbell model with $\varepsilon=0$ (see Theorem 2.2 in Section 2). Our main approach is to introduce two new parameters $\beta, b$ and to put forward a modified model depending on four parameters $\alpha, \beta, \varepsilon, b$. After obtaining the global weak solutions to this modified model, we pass to the limit $\beta \rightarrow 0_{+}$to derive the global weak solutions to the regularized model. Next, on passage to the limit $\varepsilon \rightarrow 0_{+}$, we obtain the global weak solutions to the reduced model with $\varepsilon=0$. Here, the modified model comes from a series of equations depending on $\beta$ equivalent to Equation (1.6) with a term of order $O(\beta)$. The global existence of this modified model is in the framework of the Rothe method [28], in which the crucial step is to establish a priori estimates for $\mathbf{u}$ and $\psi$. With a family of weighted Sobolev spaces depending on $\beta, b$ established, we can get 
over the difficulty introduced by the term $\left(\nabla_{x} \mathbf{u}\right) \mathbf{q} \psi$ and obtain a priori estimates for $\mathbf{u}, \psi$ for the modified model. Another especially mentioned point is to get a priori bounds for $\mathbf{C}(\psi)$. If we adopt the idea of [2], then the norm of $\mathbf{C}(\psi)$ depends on $\beta$ and then converges to $\infty$ as $\beta$ goes to zero. Here we introduce another parameter $b$ and apply the Carlson inequality to solve it. Now we expect to establish the global weak solutions to the classical Hookean dumbbell model with $(\alpha, \varepsilon)=(0,0)$ in future work.

The paper is organized as follows. In Section 2, we formulate the regularized Hookean dumbbell model and give two definitions. Our main results are also stated in this section. As a preliminary, we firstly introduce the Maxwellian $M(\mathbf{q})$ in Section 3.1. Then we establish a family of weighted Sobolev spaces, as well as trace and density results and so on for these spaces, in Section 3.2. In Section 3.3, we show some properties of the isotropic Friedrichs mollifier, and recall the Helmholtz-Stokes mollifier from $[1,2,11]$ in order to achieve the compactness of time derivatives of the velocity $\mathbf{u}$. In Section 3.4, some auxiliary assertions are given which will be useful below. Section 4 is devoted to studying a modified model by the Rothe method. The proofs of the main results are shown in Section 5. The conclusion is drawn in Section 6. Finally, we give some proofs of some lemmas in Appendix A for self-containedness.

\section{The model and main results}

In this section we formulate the regularized Hookean dumbbell model from [2] and state our main results. Throughout this paper, we suppose $\Omega \subset \mathbb{R}^{d}$ is a bounded open set with a Lipschitz-continuous boundary $\partial \Omega$, and $D=\mathbb{R}^{d}, d=2$ or 3 . $\mathbf{u}_{\alpha, \varepsilon}(\mathbf{x}, t)$ denotes the velocity field depending on the parameters $\alpha, \varepsilon, p_{\alpha, \varepsilon}(\mathbf{x}, t)$ and $\psi_{\alpha, \varepsilon}(\mathbf{x}, \mathbf{q}, t)$ are similarly defined. For the parameters $\alpha, \varepsilon \in(0,1]$, we consider the regularized Hookean dumbbell model. Mathematically, this system reads:

$$
\begin{aligned}
\frac{\partial \mathbf{u}_{\alpha, \varepsilon}}{\partial t}+ & \left(\mathbf{u}_{\alpha, \varepsilon} \cdot \nabla_{x}\right) \mathbf{u}_{\alpha, \varepsilon}-\nu \Delta_{x} \mathbf{u}_{\alpha, \varepsilon}+\nabla_{x} p_{\alpha, \varepsilon}=\nabla_{x} \cdot \tau\left(\psi_{\alpha, \varepsilon}\right) \quad \text { in } \Omega \times(0, T] \\
\nabla_{x} \cdot \mathbf{u}_{\alpha, \varepsilon}= & 0 \quad \text { in } \Omega \times(0, T], \\
\tau\left(\psi_{\alpha, \varepsilon}\right)= & k \omega\left(\mathbf{C}\left(J_{\alpha}^{x} \psi_{\alpha, \varepsilon}\right)-\rho\left(\psi_{\alpha, \varepsilon}\right) \mathbf{I}\right) \quad \text { in } \Omega \times(0, T] \\
& \frac{\partial \psi_{\alpha, \varepsilon}}{\partial t}+\left(\mathbf{u}_{\alpha, \varepsilon} \cdot \nabla_{x}\right) \psi_{\alpha, \varepsilon}+\nabla_{q} \cdot\left(\nabla_{x}\left(\mathbf{J}_{\alpha}^{x} \mathbf{u}_{\alpha, \varepsilon}\right) \mathbf{q} \psi\right) \\
= & \frac{1}{2 \lambda} \nabla_{q} \cdot\left(\nabla_{q} \psi_{\alpha, \varepsilon}+\nabla_{q} U \psi_{\alpha, \varepsilon}\right)+\varepsilon \Delta_{x} \psi_{\alpha, \varepsilon} \quad \text { in } \Omega \times D \times(0, T]
\end{aligned}
$$

where

$$
\begin{aligned}
\mathbf{C}\left(\psi_{\alpha, \varepsilon}\right) & =\int_{D}\left(\nabla_{q} U \otimes \mathbf{q}\right) \psi_{\alpha, \varepsilon}(\mathbf{x}, \mathbf{q}, t) d \mathbf{q} \\
\rho\left(\psi_{\alpha, \varepsilon}\right) & =\int_{D} \psi_{\alpha, \varepsilon}(\mathbf{x}, \mathbf{q}, t) d \mathbf{q} .
\end{aligned}
$$

$U$ in (2.5) is the Hookean potential form as (1.8). $J_{\alpha}^{x}$ in (2.3) is the isotropic Friedrichs mollifier defined as follows: Let $j$ be a non-negative function in $W^{1, \infty}\left(\mathbb{R}^{d}\right)$ vanishing outside the unit ball $B(0,1)$ and satisfying $\int_{B(0,1)} j(\mathbf{x}) d \mathbf{x}=1$ and $j(-\mathbf{x})=j(\mathbf{x})$ for all $\mathbf{x} \in B(0,1)$. For $\varphi \in L^{1}(\Omega)$ and $\alpha \in(0,1]$, the regularization of $\varphi$, denoted by $\left(J_{\alpha}^{x} \varphi\right)(\mathbf{x})$ is then defined by the convolution

$$
\left(J_{\alpha}^{x} \varphi\right)(\mathbf{x}):=\alpha^{-d} \int_{\Omega} j\left(\frac{\mathbf{x}-\mathbf{y}}{\alpha}\right) \varphi(\mathbf{y}) d \mathbf{y}, \quad \forall \mathbf{x} \in \Omega .
$$


In the same way, we can define $\mathbf{J}_{\alpha}^{x}$, the vector or tensor form of $J_{\alpha}^{x}$. For brevity, sometimes we will employ the notation of $\varphi_{\alpha}$ instead of $J_{\alpha}^{x} \varphi$.

For the system (2.1)-(2.4), the boundary and initial conditions are the following:

$$
\begin{aligned}
\mathbf{u}_{\alpha, \varepsilon} & =0 \quad \text { on } \partial \Omega \times(0, T], \\
\varepsilon \nabla_{x} \psi_{\alpha, \varepsilon} \cdot \mathbf{n} & =0 \quad \text { on } \partial \Omega \times D \times(0, T], \\
\mathbf{u}_{\alpha, \varepsilon}(\mathbf{x}, 0) & =\mathbf{u}_{0}(\mathbf{x}) \quad \forall \mathbf{x} \in \Omega, \\
\psi_{\alpha, \varepsilon}(\mathbf{x}, \mathbf{q}, 0) & =\psi_{0}(\mathbf{x}, \mathbf{q}) \geq 0 \quad \forall(\mathbf{x}, \mathbf{q}) \in \Omega \times D .
\end{aligned}
$$

Here $\mathbf{n}$ denotes the unit outward normal to the boundary $\partial \Omega$.

Now we firstly state some assumptions:

Assumptions: $\left(\mathbf{A}_{1}\right)$ : If $d=2, b \geq 8$ and if $d=3, b \geq 10$.

$\left(\mathbf{A}_{2}\right): \quad \mathbf{u}_{0} \in \mathbf{H}, \psi_{0} \in L^{2,+}\left(\Omega \times D ; 1+|\mathbf{q}|^{b}\right) \cap L^{1}(\Omega \times D ; 1+U)$ and $\int_{\Omega \times D} \psi_{0} d \mathbf{q} d \mathbf{x}=1$. Throughout this paper, we use the superscript + to imply that any function in the space is nonnegative in an almost everywhere sense.

In this paper, we employ certain fundamental spaces in the study of Navier-Stokes equations $[13,30]$ :

$$
\begin{aligned}
& \mathcal{V}:=\left\{\mathbf{v} \in \mathbf{C}_{0}^{\infty}(\Omega): \nabla_{x} \cdot \mathbf{v}=0\right\} \\
& \mathbf{H}:=\left\{\mathbf{v} \in \mathbf{L}^{2}(\Omega): \nabla_{x} \cdot \mathbf{v}=0, \mathbf{v} \cdot \mathbf{n}=0\right\} \\
& \mathbf{V}:=\left\{\mathbf{v} \in \mathbf{H}_{0}^{1}(\Omega): \nabla_{x} \cdot \mathbf{v}=0\right\}
\end{aligned}
$$

It is well-known that $\mathcal{V}$ is dense both in $\mathbf{H}$ and $\mathbf{V}$. Moreover we construct the following Hilbert spaces depending on the parameter $b \geq 0$ :

$$
\begin{aligned}
& X_{b}:=\left\{\varphi \in L_{\mathrm{loc}}^{1}(\Omega \times D):\|\varphi\|_{X_{b}}<\infty\right\}, \\
& Y_{b}:=\left\{\varphi \in L_{\mathrm{loc}}^{1}(\Omega \times D):\|\varphi\|_{Y_{b}}<\infty\right\}, \\
& X_{b, 0}:=\left\{\varphi \in L_{\mathrm{loc}}^{1}(\Omega \times D):\|\varphi\|_{X_{b, 0}}<\infty\right\},
\end{aligned}
$$

where

$$
\begin{aligned}
& \|\varphi\|_{X_{b}}:=\left\{\int_{\Omega \times D}\left(1+|\mathbf{q}|^{b}\right)\left[|\varphi|^{2}+\left|\nabla_{x} \varphi\right|^{2}+\left|\nabla_{q} \varphi\right|^{2}\right] d \mathbf{q} d \mathbf{x}\right\}^{\frac{1}{2}} \\
& \|\varphi\|_{Y_{b}}:=\left\{\int_{\Omega \times D}\left[\left(1+|\mathbf{q}|^{b+4}\right)|\varphi|^{2}+\left(1+|\mathbf{q}|^{b}\right)\left|\nabla_{x} \varphi\right|^{2}+\left(1+|\mathbf{q}|^{b+2}\right)\left|\nabla_{q} \varphi\right|^{2}\right] d \mathbf{q} d \mathbf{x}\right\}^{\frac{1}{2}} \\
& \|\varphi\|_{X_{b, 0}}:=\left\{\int_{\Omega \times D}\left(1+|\mathbf{q}|^{b}\right)\left[|\varphi|^{2}+\left|\nabla_{q} \varphi\right|^{2}\right] d \mathbf{q} d \mathbf{x}\right\}^{\frac{1}{2}}
\end{aligned}
$$

Analogously, we introduce $Y_{b, 0}$.

Furthermore, we require the following spaces for the test functions. Denote by $\mathcal{Y}_{b}$ the completion of $C_{0}^{2}\left(-T, T ; Y_{b}\right)$ in the norm $\|\cdot\|_{\mathcal{Y}_{b}}$ defined by

$$
\begin{aligned}
\|\varphi\|_{\mathcal{Y}_{b}}:= & \|\varphi\|_{L^{1}\left(0, T ; Y_{b}\right)}+\left\|\left(1+|\mathbf{q}|^{b}\right)^{\frac{1}{2}} \nabla_{x} \varphi\right\|_{L^{\frac{4}{4-d}}\left(0, T ; L^{2}(\Omega \times D)\right)} \\
& +\left\|\left(1+|\mathbf{q}|^{b}\right)^{\frac{1}{2}} \nabla_{q} \varphi\right\|_{L^{2}\left(0, T ; L^{2}(\Omega \times D)\right)}+\left\|\left.\mathbf{q}\right|^{\frac{b}{2}-1} \varphi\right\|_{L^{2}\left(0, T ; L^{2}(\Omega \times D)\right)} \\
& +\left\|\left(1+|\mathbf{q}|^{b}\right)^{\frac{1}{2}} \frac{\partial \varphi}{\partial t}\right\|_{L^{1}\left(0, T ; L^{2}(\Omega \times D)\right)} .
\end{aligned}
$$


Denote by $\mathcal{Z}_{b}$ the completion of $C_{0}^{2}\left(-T, T ; \mathcal{K}^{0}\right)$ in the norm $\|\cdot\|_{\mathcal{Z}_{b}}$ defined by

$$
\begin{aligned}
\|\varphi\|_{\mathcal{Z}_{b}}:= & \|\varphi\|_{L^{1}\left(0, T ; Y_{b, 0}\right)}+\left\|\left(1+|\mathbf{q}|^{b}\right)^{\frac{1}{2}} \nabla_{x} \varphi\right\|_{L^{\frac{4}{4-d}}\left(0, T ; L^{2}(\Omega \times D)\right)} \\
& +\left\||\mathbf{q}|^{\frac{b}{2}-1} \varphi\right\|_{L^{2}\left(0, T ; L^{2}(\Omega \times D)\right)} \\
& +\left\|\left(1+|\mathbf{q}|^{b}\right)^{\frac{1}{2}} \nabla_{q} \varphi\right\|_{L^{2}\left(0, T ; L^{2}(\Omega \times D)\right)}+\left\|\left(1+|\mathbf{q}|^{b}\right)^{\frac{1}{2}} \frac{\partial \varphi}{\partial t}\right\|_{L^{1}\left(0, T ; L^{2}(\Omega \times D)\right)},
\end{aligned}
$$

where $\mathcal{K}^{0}:=C_{0}^{\infty}(\Omega \times D)$.

Next we will show the precise definition of a global weak solution to the regularized Hookean dumbbell model (2.1)-(2.4) together with (2.8)-(2.11).

Definition 2.1. Given $\alpha, \varepsilon \in(0,1]$ and $b$ satisfying Assumption $\left(A_{1}\right), a$ pair of functions $\left(\mathbf{u}_{\alpha, \varepsilon, b}, \psi_{\alpha, \varepsilon, b}\right)$ is called a weak solution to the problem (2.1)-(2.4) together with (2.8)-(2.11) provided that $\mathbf{u}_{\alpha, \varepsilon, b} \in L^{\infty}\left(0, T ; \mathbf{L}^{2}(\Omega)\right) \cap$ $L^{2}(0, T ; \mathbf{V}) \cap W^{1, \frac{4}{d}}\left(0, T ; \mathbf{V}^{\prime}\right), \quad \psi_{\alpha, \varepsilon, b} \in L^{2}\left(0, T ; X_{b}^{+}\right) \cap L^{\infty}\left(0, T ; L^{2}\left(\Omega \times D ; 1+|\mathbf{q}|^{b}\right)\right)$, $\mathbf{J}_{\alpha}^{x} \mathbf{u}_{\alpha, \varepsilon, b} \in L^{\infty}\left(0, T ; \mathbf{W}^{1, \infty}(\Omega)\right), \mathbf{C}\left(\psi_{\alpha, \varepsilon, b}\right) \in L^{\infty}\left(0, T ; \mathbf{L}^{2}(\Omega)\right)$, and

$$
\begin{aligned}
\int_{0}^{T}< & \frac{\partial \mathbf{u}_{\alpha, \varepsilon, b}}{\partial t}, \mathbf{w}>d t+\int_{0}^{T} \int_{\Omega}\left[\left[\left(\mathbf{u}_{\alpha, \varepsilon, b} \cdot \nabla_{x}\right) \mathbf{u}_{\alpha, \varepsilon, b}\right] \cdot \mathbf{w}+\nu \nabla_{x} \mathbf{u}_{\alpha, \varepsilon, b}: \nabla_{x} \mathbf{w}\right] d \mathbf{x} d t \\
= & -\kappa \omega \int_{0}^{T} \int_{\Omega} \mathbf{C}\left(\psi_{\alpha, \varepsilon, b}\right): \nabla_{x} \mathbf{w}_{\alpha} d \mathbf{x} d t, \quad \forall \mathbf{w} \in L^{\frac{4}{4-d}}(0, T ; \mathbf{V}) \\
& -\int_{0}^{T} \int_{\Omega \times D}\left(1+|\mathbf{q}|^{b}\right) \psi_{\alpha, \varepsilon, b} \frac{\partial \varphi}{\partial t} d \mathbf{q} d \mathbf{x} d t-\int_{\Omega \times D}\left(1+|\mathbf{q}|^{b}\right) \psi_{0}(\cdot, \cdot) \varphi(\cdot, \cdot, 0) d \mathbf{q} d \mathbf{x} \\
& +\int_{0}^{T} \int_{\Omega \times D}\left[\frac{1}{2 \lambda} \nabla_{q} \psi_{\alpha, \varepsilon, b}-\left[\nabla_{x}\left(\mathbf{J}_{\alpha}^{x} \mathbf{u}_{\alpha, \varepsilon, b}\right) \mathbf{q}\right] \psi_{\alpha, \varepsilon, b}+\frac{1}{2 \lambda} \mathbf{q} \psi_{\alpha, \varepsilon, b}\right] \\
& \cdot \nabla_{q}\left(\left(1+|\mathbf{q}|^{b}\right) \varphi\right) d \mathbf{q} d \mathbf{x} d t \\
& +\int_{0}^{T} \int_{\Omega \times D}\left(1+|\mathbf{q}|^{b}\right)\left[\varepsilon \nabla_{x} \psi_{\alpha, \varepsilon, b}-\mathbf{u}_{\alpha, \varepsilon, b} \psi_{\alpha, \varepsilon, b}\right] \cdot \nabla_{x} \varphi d \mathbf{q} d \mathbf{x} d t=0, \quad \forall \varphi \in \mathcal{Y}_{b} \\
& \mathbf{u}_{\alpha, \varepsilon, b}(\cdot, 0)=\mathbf{u}_{0}(\cdot) .
\end{aligned}
$$

Similarly, we also give the definition of a global weak solution to the reduced Hookean dumbbell model (2.1)-(2.4) together with (2.8)-(2.11) with $\varepsilon=0$.

Definition 2.2. Given $\alpha \in(0,1]$ and $b$ satisfying Assumption $\left(A_{1}\right)$, a pair of functions $\left(\mathbf{u}_{\alpha, b}, \psi_{\alpha, b}\right)$ is called a weak solution to the reduced Hookean dumbbell model (2.1)-(2.4) together with (2.8)-(2.11) with $\varepsilon=0$ provided that $\mathbf{u}_{\alpha, b} \in$ $L^{\infty}\left(0, T ; \mathbf{L}^{2}(\Omega)\right) \cap L^{2}(0, T ; \mathbf{V}) \cap W^{1, \frac{4}{d}}\left(0, T ; \mathbf{V}^{\prime}\right), \psi_{\alpha, b} \in L^{2}\left(0, T ; X_{b, 0}^{+}\right) \cap L^{\infty}\left(0, T ; L^{2}(\Omega \times\right.$ $\left.\left.D ; 1+|\mathbf{q}|^{b}\right)\right), \mathbf{J}_{\alpha}^{x} \mathbf{u}_{\alpha, b} \in L^{\infty}\left(0, T ; \mathbf{W}^{1, \infty}(\Omega)\right), \mathbf{C}\left(\psi_{\alpha, b}\right) \in L^{\infty}\left(0, T ; \mathbf{L}^{2}(\Omega)\right)$, and

$$
\begin{aligned}
\int_{0}^{T}< & \frac{\partial \mathbf{u}_{\alpha, b}}{\partial t}, \mathbf{w}>d t+\int_{0}^{T} \int_{\Omega}\left[\left[\left(\mathbf{u}_{\alpha, b} \cdot \nabla_{x}\right) \mathbf{u}_{\alpha, b}\right] \cdot \mathbf{w}+\nu \nabla_{x} \mathbf{u}_{\alpha, b}: \nabla_{x} \mathbf{w}\right] d \mathbf{x} d t \\
= & -\kappa \omega \int_{0}^{T} \int_{\Omega} \mathbf{C}\left(\psi_{\alpha, b}\right): \nabla_{x} \mathbf{w}_{\alpha} d \mathbf{x} d t, \quad \forall \mathbf{w} \in L^{\frac{4}{4-d}}(0, T ; \mathbf{V}) \\
& -\int_{0}^{T} \int_{\Omega \times D}\left(1+|\mathbf{q}|^{b}\right) \psi_{\alpha, b} \frac{\partial \varphi}{\partial t} d \mathbf{q} d \mathbf{x} d t-\int_{\Omega \times D}\left(1+|\mathbf{q}|^{b}\right) \psi_{0}(\cdot, \cdot) \varphi(\cdot, \cdot, 0) d \mathbf{q} d \mathbf{x}
\end{aligned}
$$




$$
\begin{aligned}
& +\int_{0}^{T} \int_{\Omega \times D}\left[\frac{1}{2 \lambda} \nabla_{q} \psi_{\alpha, b}-\left[\nabla_{x}\left(\mathbf{J}_{\alpha}^{x} \mathbf{u}_{\alpha, b}\right) \mathbf{q}\right] \psi_{\alpha, b}+\frac{1}{2 \lambda} \mathbf{q} \psi_{\alpha, b}\right] \cdot \nabla_{q}\left(\left(1+|\mathbf{q}|^{b}\right) \varphi\right) d \mathbf{q} d \mathbf{x} d t \\
& -\int_{0}^{T} \int_{\Omega \times D}\left(1+|\mathbf{q}|^{b}\right)\left(\mathbf{u}_{\alpha, b} \psi_{\alpha, b}\right) \cdot \nabla_{x} \varphi d \mathbf{q} d \mathbf{x} d t=0, \quad \forall \varphi \in \mathcal{Z}_{b} \\
& \mathbf{u}_{\alpha, b}(\cdot, 0)=\mathbf{u}_{0}(\cdot) .
\end{aligned}
$$

Finally we state our main results.

Proposition 2.3. $\psi_{\alpha, \varepsilon, b}$ and $\psi_{\alpha, b}$ in Definition 2.1 and 2.2 belong to $L^{\infty}\left(0, T ; L^{1}(\Omega \times\right.$ $D ; 1+U))$. Moreover, $\forall t \in(0, T)$, we have

$$
\int_{\Omega \times D} \psi_{\alpha, \varepsilon, b}(\mathbf{x}, \mathbf{q}, t) d \mathbf{q} d \mathbf{x}=\int_{\Omega \times D} \psi_{\alpha, b}(\mathbf{x}, \mathbf{q}, t) d \mathbf{q} d \mathbf{x}=\int_{\Omega \times D} \psi_{0}(\mathbf{x}, \mathbf{q}) d \mathbf{q} d \mathbf{x}=1 .
$$

Theorem 2.1. Suppose Assumptions $\left(A_{1}\right)-\left(A_{2}\right)$. Then, for given $\alpha, \varepsilon \in(0,1]$, the regularized Hookean dumbbell model (2.1)-(2.4) together with (2.8)-(2.11) possesses a weak solution $\left(\mathbf{u}_{\alpha, \varepsilon, b}, \psi_{\alpha, \varepsilon, b}\right)$ in the sense of Definition 2.1.

THEOREM 2.2. Let assumptions $\left(A_{1}\right)-\left(A_{2}\right)$ be fulfilled and $\alpha \in(0,1]$. Then, the reduced Hookean dumbbell model (2.1)-(2.4) together with (2.8)-(2.11) with $\varepsilon=0$ possesses a weak solution $\left(\mathbf{u}_{\alpha, b}, \psi_{\alpha, b}\right)$ in the sense of Definition 2.2.

Moreover, the subsequence $\left\{\mathbf{u}_{\alpha, \varepsilon, b}, \psi_{\alpha, \varepsilon, b}\right\}_{\varepsilon}$ converges to the solution $\left(\mathbf{u}_{\alpha, b}, \psi_{\alpha, b}\right)$ in the following sense:

$$
\begin{aligned}
&\left(1+|\mathbf{q}|^{b}\right)^{\frac{1}{2}} \psi_{\alpha, \varepsilon, b} \stackrel{*}{\rightarrow}\left(1+|\mathbf{q}|^{b}\right)^{\frac{1}{2}} \psi_{\alpha, b} \text { in } L^{\infty}\left(0, T ; L^{2}(\Omega \times D)\right), \\
& \varepsilon\left(1+|\mathbf{q}|^{b}\right)^{\frac{1}{2}} \nabla_{x} \psi_{\alpha, \varepsilon, b} \rightarrow 0 \text { in } L^{2}\left(0, T ; \mathbf{L}^{2}(\Omega \times D)\right), \\
&\left(1+|\mathbf{q}|^{b}\right)^{\frac{1}{2}} \nabla_{q} \psi_{\alpha, \varepsilon, b} \rightarrow\left(1+|\mathbf{q}|^{b}\right)^{\frac{1}{2}} \nabla_{q} \psi_{\alpha, b} \text { in } L^{2}\left(0, T ; \mathbf{L}^{2}(\Omega \times D)\right), \\
& \mathbf{C}\left(\psi_{\alpha, \varepsilon, b}\right) \stackrel{*}{\rightarrow} \mathbf{C}\left(\psi_{\alpha, b}\right) \text { in } L^{\infty}\left(0, T ; \mathbf{L}^{2}(\Omega)\right), \\
& \mathbf{u}_{\alpha, \varepsilon, b} \stackrel{*}{\rightarrow} \mathbf{u}_{\alpha, b} \text { in } L^{\infty}\left(0, T ; \mathbf{L}^{2}(\Omega)\right), \\
& \mathbf{u}_{\alpha, \varepsilon, b} \rightarrow \mathbf{u}_{\alpha, b} \text { in } L^{2}(0, T ; \mathbf{V}), \\
& \mathbf{S}_{\gamma} \frac{\partial \mathbf{u}_{\alpha, \varepsilon, b}}{\partial t} \rightarrow \mathbf{S}_{\gamma} \frac{\partial \mathbf{u}_{\alpha, b}}{\partial t} \text { in } L^{\frac{4}{\alpha}(0, T ; \mathbf{V}),} \\
& \mathbf{u}_{\alpha, \varepsilon, b} \rightarrow \mathbf{u}_{\alpha, b} \text { in } L^{2}\left(0, T ; \mathbf{L}^{r}(\Omega)\right), \\
& \mathbf{J}_{\alpha}^{x} \mathbf{u}_{\alpha, \varepsilon, b} \rightarrow \mathbf{J}_{\alpha}^{x} \mathbf{u}_{\alpha, b} \text { in } L^{2}\left(0, T ; \mathbf{W}^{1, \infty}(\Omega)\right), \\
& \mathbf{J}_{\alpha}^{x} \mathbf{u}_{\alpha, \varepsilon, b} \stackrel{*}{\rightarrow} \mathbf{J}_{\alpha}^{x} \mathbf{u}_{\alpha, b} \text { in } L^{\infty}\left(0, T ; \mathbf{W}^{1, \infty}(\Omega)\right),
\end{aligned}
$$

as $\varepsilon \rightarrow 0_{+}$, where $r \in[1, \infty)$ if $d=2$ and $r \in[1,6)$ if $d=3$.

REMARK 2.4. The regularized Hookean dumbbell model depending on $\alpha, \varepsilon$ also corresponds to a deterministic constitutive equation. The brief derivation is given as follows.

Set $\mathbf{C}_{\alpha, \varepsilon}(\mathbf{x}, t):=\mathbf{C}\left(\psi_{\alpha, \varepsilon}\right)(\mathbf{x}, t), \rho_{\alpha, \varepsilon}(\mathbf{x}, t):=\rho\left(\psi_{\alpha, \varepsilon}\right)(\mathbf{x}, t)$ and assume that $\psi_{\alpha, \varepsilon}$ and $\left|\nabla_{q} \psi_{\alpha, \varepsilon}\right|$ decay to zero sufficiently fast as $\mathbf{q} \rightarrow \infty$. Then multiplying (2.4) by $\mathbf{q q}^{T}$ and integrating over $D$ yields that

$$
\begin{gathered}
\frac{\partial \mathbf{C}_{\alpha, \varepsilon}}{\partial t}+\left(\mathbf{u}_{\alpha, \varepsilon} \cdot \nabla_{x}\right) \mathbf{C}_{\alpha, \varepsilon}-\nabla_{x}\left(\mathbf{J}_{\alpha}^{x} \mathbf{u}_{\alpha, \varepsilon}\right) \mathbf{C}_{\alpha, \varepsilon}-\mathbf{C}_{\alpha, \varepsilon}\left[\nabla_{x}\left(\mathbf{J}_{\alpha}^{x} \mathbf{u}_{\alpha, \varepsilon}\right)\right]^{T} \\
-\varepsilon \Delta_{x} \mathbf{C}_{\alpha, \varepsilon}+\frac{1}{\lambda} \mathbf{C}_{\alpha, \varepsilon}=\frac{\rho_{\alpha, \varepsilon}}{\lambda} \mathbf{I} \quad \text { in } \Omega \times(0, T] .
\end{gathered}
$$


Similarly, integrating (2.4) over D yields that

$$
\frac{\partial \rho_{\alpha, \varepsilon}}{\partial t}-\varepsilon \Delta_{x} \rho_{\alpha, \varepsilon}+\left(\mathbf{u}_{\alpha, \varepsilon} \cdot \nabla_{x}\right) \rho_{\alpha, \varepsilon}=0 \quad \text { in } \Omega \times(0, T] .
$$

On the other hand, it follows from (2.1) and (2.3) that

$$
\begin{aligned}
& \frac{\partial \mathbf{u}_{\alpha, \varepsilon}}{\partial t}+\left(\mathbf{u}_{\alpha, \varepsilon} \cdot \nabla_{x}\right) \mathbf{u}_{\alpha, \varepsilon}-\nu \Delta_{x} \mathbf{u}_{\alpha, \varepsilon}+\nabla_{x}\left(p_{\alpha, \varepsilon}+k \omega \rho_{\alpha, \varepsilon}\right) \\
= & k \omega \nabla_{x} \cdot\left[\mathbf{J}_{\alpha}^{x}\left(\mathbf{C}_{\alpha, \varepsilon}\right)\right] \quad \text { in } \Omega \times(0, T] .
\end{aligned}
$$

Then, (2.37)-(2.39) and (2.2) are finally closed by $\mathbf{u}_{\alpha, \varepsilon}, \mathbf{C}_{\alpha, \varepsilon}, \rho_{\alpha, \varepsilon}$. In the special case $\alpha=\varepsilon=0$, it is just the well-known Oldroyd-B model.

\section{Preliminaries}

In this section and the next section, we first state an important assumption: Assumption $\left(\mathrm{A}_{3}\right): \beta>0$.

3.1. The Maxwellian. Now we adopt a normalized Maxwellian distribution induced by $U$ as follows:

$$
M(\mathbf{q})=\frac{e^{-U(\mathbf{q})}}{\int_{D} e^{-U(\mathbf{q})} d \mathbf{q}}=\frac{e^{-\frac{1}{2}|\mathbf{q}|^{2}}}{\int_{D} e^{-\frac{1}{2}|\mathbf{q}|^{2}} d \mathbf{q}} .
$$

From the definition of $M$, we can obtain the following properties.

P1: For any given $\beta>0$,

$$
M^{\beta} \nabla_{q} M^{-\beta}=-M^{-\beta} \nabla_{q} M^{\beta}=\beta \nabla_{q} U=\beta \mathbf{q} .
$$

P2: Define $Z:=\int_{D} e^{-\frac{1}{2}|\mathbf{q}|^{2}} d \mathbf{q}$ the gamma function $\Gamma(a):=\int_{0}^{\infty} x^{a-1} e^{-x} d x$, and $\sigma(d):=\frac{d \pi^{d / 2}}{\Gamma\left(\frac{d}{2}+1\right)}$, which is the surface area of the unit sphere $B(\mathbf{0}, 1)$ in $\mathbb{R}^{d}$. Then

$$
\begin{aligned}
Z & =\int_{0}^{\infty} \int_{\partial B(\mathbf{0}, 1)} e^{-\frac{1}{2} r^{2}} r^{d-1} d \theta d r \\
& =2^{\frac{d}{2}-1} \sigma(d) \Gamma\left(\frac{d}{2}\right)= \begin{cases}2 \pi, & \text { if } d=2, \\
(2 \pi)^{\frac{3}{2}}, & \text { if } d=3 .\end{cases}
\end{aligned}
$$

It is obvious that $\forall \beta>0$,

$$
Z^{\beta}>1
$$

which implies that

$$
\frac{1}{M^{\beta_{2}}}>\frac{1}{M^{\beta_{1}}}>1, \quad \forall \beta_{2}>\beta_{1}>0 .
$$

P3: For any given $\beta>0$ and $s \in \mathbb{R}$,

$$
\begin{aligned}
\int_{D} M^{\beta}|\mathbf{q}|^{s} d \mathbf{q} & =Z^{-\beta} \int_{D} e^{-\frac{\beta}{2}|\mathbf{q}|^{2}}|\mathbf{q}|^{s} d \mathbf{q} \\
& =\frac{1}{2} \sigma(d) Z^{-\beta}\left(\frac{2}{\beta}\right)^{\frac{d+s}{2}} \Gamma\left(\frac{d+s}{2}\right) .
\end{aligned}
$$

Hence, when $d+s>0$,

$$
\int_{D} M^{\beta}|\mathbf{q}|^{s} d \mathbf{q}<\infty
$$


3.2. Weighted Sobolev spaces. In this subsection we introduce the following spaces for any given $b$ and $\beta$ satisfying Assumptions $\left(A_{1}\right)$ and $\left(A_{3}\right)$, respectively:

$$
\begin{aligned}
X_{b}^{\beta} & :=\left\{\varphi \in L_{\mathrm{loc}}^{1}(\Omega \times D):\|\varphi\|_{X_{b}^{\beta}}<\infty\right\}, \\
Y_{b}^{\beta} & :=\left\{\varphi \in L_{\mathrm{loc}}^{1}(\Omega \times D):\|\varphi\|_{Y_{b}^{\beta}}<\infty\right\}, \\
Z_{b}^{\beta} & :=\left\{\varphi \in L_{\mathrm{loc}}^{1}(\Omega \times D):\|\varphi\|_{Z_{b}^{\beta}}<\infty\right\},
\end{aligned}
$$

where

$$
\begin{aligned}
& \|\varphi\|_{X_{b}^{\beta}}:=\left\{\int_{\Omega \times D}\left(1+|\mathbf{q}|^{b}\right)\left[\frac{|\varphi|^{2}}{M^{\beta}}+\frac{\left|\nabla_{x} \varphi\right|^{2}}{M^{\beta}}+M^{\beta}\left|\nabla_{q} \frac{\varphi}{M^{\beta}}\right|^{2}\right] d \mathbf{q} d \mathbf{x}\right\}^{\frac{1}{2}}, \\
& \|\varphi\|_{Y_{b}^{\beta}} \\
:= & \left\{\int_{\Omega \times D}\left[\left(1+|\mathbf{q}|^{b+4}\right) \frac{|\varphi|^{2}}{M^{\beta}}+\left(1+|\mathbf{q}|^{b}\right) \frac{\left|\nabla_{x} \varphi\right|^{2}}{M^{\beta}}+\left(1+|\mathbf{q}|^{b+2}\right) M^{\beta}\left|\nabla_{q} \frac{\varphi}{M^{\beta}}\right|^{2}\right] d \mathbf{q} d \mathbf{x}\right\}^{\frac{1}{2}}, \\
& \|\varphi\|_{Z_{b}^{\beta}}:=\left\{\int_{\Omega \times D}\left(1+|\mathbf{q}|^{b}\right)\left[\left(1+|\mathbf{q}|^{2}\right) \frac{|\varphi|^{2}}{M^{\beta}}+\frac{\left|\nabla_{x} \varphi\right|^{2}}{M^{\beta}}+M^{\beta}\left|\nabla_{q} \frac{\varphi}{M^{\beta}}\right|^{2}\right] d \mathbf{q} d \mathbf{x}\right\}^{\frac{1}{2}} .
\end{aligned}
$$

Similarly to the space $X_{b, 0}$ defined in (2.17) and (2.19), we introduce $X_{b, 0}^{\beta}, Y_{b, 0}^{\beta}, Z_{b, 0}^{\beta}$. Note that $\varphi \in X_{b}^{\beta}$ if and only if $\frac{\varphi}{M^{\beta}} \in H^{1}\left(\Omega \times D ;\left(1+|\mathbf{q}|^{b}\right) M^{\beta}\right)$. Then $X_{b}^{\beta}$ is a Hilbert space, since the space $H^{1}\left(\Omega \times D ;\left(1+|\mathbf{q}|^{b}\right) M^{\beta}\right)$ is a Hilbert space. Analogously, the other weighted spaces defined here are all Hilbert spaces. Moreover, from (3.5), we have $M^{\beta} /\left(1+|\mathbf{q}|^{b}\right), M^{\beta} U /\left(1+|\mathbf{q}|^{b}\right) \in Y_{b}^{\beta}$ under the assumptions $\left(\mathrm{A}_{1}\right)$ and $\left(\mathrm{A}_{3}\right)$.

REMARK 3.1. From the above definitions of a family of weighted Sobolev spaces depending on parameters $\beta$ and $b$, it is not difficult to get the relations among them: $Y_{b}^{\beta} \subset Z_{b}^{\beta} \subset X_{b}^{\beta}$ and $Y_{b, 0}^{\beta} \subset Z_{b, 0}^{\beta} \subset X_{b, 0}^{\beta}$. Moreover, $X_{b}^{\beta_{2}} \subset X_{b}^{\beta_{1}} \subset X_{b}, Y_{b}^{\beta_{2}} \subset Y_{b}^{\beta_{1}} \subset Y_{b}$, and $Z_{b}^{\beta_{2}} \subset Z_{b}^{\beta_{1}}$ if $\beta_{2}>\beta_{1}>0$.

We now simply write $B_{R}:=B(\mathbf{0}, R)$ for any $R>0$. From Lemma 3.2 in [1], we have that $\varphi$ in $X_{0,0}^{1}$ possesses the decay property

$$
\lim _{R \rightarrow \infty} R^{\delta} \int_{\Omega \times \partial B_{R}}|\varphi| d S(\mathbf{q}) d \mathbf{x}=0 \quad \forall \delta \geq 0
$$

where $d S(\mathbf{q})$ indicates the $(d-1)$-dimensional area element in $\partial B_{R}$. We can show that $\varphi$ in $Y_{b}^{\beta}$ also satisfies (3.12) similarly. However, this trace theorem is not enough for the wellposedness analysis of our problems. So we will give another trace result which needs the following lemma in the proof.

Lemma 3.1. Let $R \geq 1$ and assume $\varphi \in L^{2}\left(\Omega ; H^{1}\left(B_{R}\right)\right)$. Then for some constant $C:=C(d)$,

$$
\|\varphi\|_{L^{2}\left(\Omega ; L^{2}\left(\partial B_{R}\right)\right)} \leq C\|\varphi\|_{L^{2}\left(\Omega ; H^{1}\left(B_{R}\right)\right)} .
$$

Proof. Recalling the divergence theorem, we have

$$
\int_{\Omega \times B_{R}} \nabla_{q} \cdot \frac{|\varphi|^{2} \mathbf{q}}{|\mathbf{q}|} d \mathbf{q} d \mathbf{x}=\int_{\Omega \times \partial B_{R}} \frac{|\varphi|^{2} \mathbf{q}}{|\mathbf{q}|} \cdot \mathbf{n} d S(\mathbf{q}) d \mathbf{x}=\int_{\Omega \times \partial B_{R}}|\varphi|^{2} d S(\mathbf{q}) d \mathbf{x} .
$$


By using the Cauchy inequality, we obtain

$$
\begin{aligned}
\int_{\Omega \times B_{R}} \nabla_{q} \cdot \frac{|\varphi|^{2} \mathbf{q}}{|\mathbf{q}|} d \mathbf{q} d \mathbf{x} & =\int_{\Omega \times B_{R}}\left(2 \varphi \nabla_{q} \varphi \cdot \frac{\mathbf{q}}{|\mathbf{q}|}+(d-1) \frac{|\varphi|^{2}}{|\mathbf{q}|}\right) d \mathbf{q} d \mathbf{x} \\
& \leq\|\varphi\|_{L^{2}\left(\Omega ; H^{1}\left(B_{R}\right)\right)}^{2}+(d-1) \int_{\Omega \times B_{R}} \frac{|\varphi|^{2}}{|\mathbf{q}|} d \mathbf{q} d \mathbf{x} .
\end{aligned}
$$

Note that

$$
\int_{\Omega \times B_{R}} \frac{|\varphi|^{2}}{|\mathbf{q}|} d \mathbf{q} d \mathbf{x} \leq \int_{\Omega \times B_{1}} \frac{|\varphi|^{2}}{|\mathbf{q}|} d \mathbf{q} d \mathbf{x}+\int_{\Omega \times B_{R}}|\varphi|^{2} d \mathbf{q} d \mathbf{x} .
$$

Application of the Hölder inequality and the imbedding $H^{1}\left(B_{1}\right) \hookrightarrow L^{6}\left(B_{1}\right)$ yields

$$
\begin{aligned}
\int_{\Omega \times B_{1}} \frac{|\varphi|^{2}}{|\mathbf{q}|} d \mathbf{q} d \mathbf{x} & \leq \int_{\Omega}\left(\int_{B_{1}}|\varphi|^{6} d \mathbf{q}\right)^{\frac{1}{3}}\left(\int_{B_{1}}|\mathbf{q}|^{-\frac{3}{2}} d \mathbf{q}\right)^{\frac{2}{3}} d \mathbf{x} \\
& \leq C(d) \int_{\Omega}\|\varphi\|_{L^{6}\left(B_{1}\right)}^{2} d \mathbf{x} \\
& \leq C(d)\|\varphi\|_{L^{2}\left(\Omega ; H^{1}\left(B_{1}\right)\right) .}^{2}
\end{aligned}
$$

Combining the above results, we can obtain (3.1).

From Lemma 3.1, we can see that the constant $C$ is independent of $R$ when $R \geq 1$. Then an application of Lemma 3.1 yields the following trace theorem.

Lemma 3.2. Under the Assumptions $\left(A_{1}\right)$ and $\left(A_{3}\right)$, we have $\forall \varphi \in Y_{b}^{\beta}$,

$$
\lim _{R \rightarrow \infty} R^{\delta} \int_{\Omega \times \partial B_{R}} \frac{|\varphi|^{2}}{M^{\beta}} d S(\mathbf{q}) d \mathbf{x}=0 \quad \forall \delta<b+2 .
$$

Proof. Define $\tilde{\varphi}:=|\mathbf{q}|^{\frac{b}{2}+1} M^{-\frac{\beta}{2}} \varphi$. Then

$$
\int_{\Omega \times D}|\tilde{\varphi}|^{2} d \mathbf{q} d \mathbf{x}=\int_{\Omega \times D}|\mathbf{q}|^{b+2} \frac{|\varphi|^{2}}{M^{\beta}} d \mathbf{q} d \mathbf{x} \leq \int_{\Omega \times D}\left(1+|\mathbf{q}|^{b+4}\right) \frac{|\varphi|^{2}}{M^{\beta}} d \mathbf{q} d \mathbf{x} .
$$

From (3.2), we have

$$
\begin{aligned}
\int_{\Omega \times D}\left|\nabla_{q} \tilde{\varphi}\right|^{2} d \mathbf{q} d \mathbf{x} & =\int_{\Omega \times D}\left|\nabla_{q}\left(|\mathbf{q}|^{\frac{b}{2}+1} \frac{\varphi}{M^{\frac{\beta}{2}}}\right)\right|^{2} d \mathbf{q} d \mathbf{x} \\
& =\left.\left.\int_{\Omega \times D}\left|\nabla_{q}\left(|\mathbf{q}|^{\frac{b}{2}+1} M^{\frac{\beta}{2}}\right) \frac{\varphi}{M^{\beta}}+\right| \mathbf{q}\right|^{\frac{b}{2}+1} M^{\frac{\beta}{2}} \nabla_{q} \frac{\varphi}{M^{\beta}}\right|^{2} d \mathbf{q} d \mathbf{x} \\
& \leq C \int_{\Omega \times D}\left(|\mathbf{q}|^{b} \frac{|\varphi|^{2}}{M^{\beta}}+|\mathbf{q}|^{b+4} \frac{|\varphi|^{2}}{M^{\beta}}+|\mathbf{q}|^{b+2} M^{\beta}\left|\nabla_{q} \frac{\varphi}{M^{\beta}}\right|^{2}\right) d \mathbf{q} d \mathbf{x} .
\end{aligned}
$$

From the above two relations we conclude that $\tilde{\varphi} \in L^{2}\left(\Omega ; H^{1}(D)\right)$ if $\varphi \in Y_{b}^{\beta}$. Let $R \geq 1$. Then Lemma 3.1 implies that

$$
\|\tilde{\varphi}\|_{L^{2}\left(\Omega ; L^{2}\left(\partial B_{R}\right)\right)} \leq C\|\tilde{\varphi}\|_{L^{2}\left(\Omega ; H^{1}\left(B_{R}\right)\right)} \leq C\|\tilde{\varphi}\|_{L^{2}\left(\Omega ; H^{1}(D)\right)} \leq C\|\varphi\|_{Y_{b}^{\beta}}
$$

where the constant $C$ is independent of $R$. Moreover, we observe that

$$
\|\tilde{\varphi}\|_{L^{2}\left(\Omega ; L^{2}\left(\partial B_{R}\right)\right)}^{2}=\int_{\Omega \times \partial B_{R}}|\mathbf{q}|^{b+2} \frac{|\varphi|^{2}}{M^{\beta}} d S(\mathbf{q}) d \mathbf{x} .
$$


Hence, for any $\delta<b+2$,

$$
\lim _{R \rightarrow \infty} R^{\delta} \int_{\Omega \times \partial B_{R}} \frac{|\varphi|^{2}}{M^{\beta}} d S(\mathbf{q}) d \mathbf{x}=C \lim _{R \rightarrow \infty} \frac{1}{R^{b+2-\delta}}\|\varphi\|_{Y_{b}^{\beta}}^{2}=0 .
$$

As a corollary to Lemma 3.2 , we can easily show that (3.12) is also true for the space $Y_{b}^{\beta}$. The proof is given below.

Lemma 3.3. Under the Assumptions $\left(A_{1}\right)$ and $\left(A_{3}\right)$, we have $\forall \varphi \in Y_{b}^{\beta}$,

$$
\lim _{R \rightarrow \infty} R^{\delta} \int_{\Omega \times \partial B_{R}}|\varphi| d S(\mathbf{q}) d \mathbf{x}=0, \quad \forall \delta \geq 0 .
$$

Proof. Let $|\Omega|$ be the volume of $\Omega$. By the Hölder inequality, we have

$$
\begin{aligned}
R^{\delta} \int_{\Omega \times \partial B_{R}}|\varphi| d S(\mathbf{q}) d \mathbf{x} & \leq R^{\delta}\left(\int_{\Omega \times \partial B_{R}}|\varphi|^{2} d S(\mathbf{q}) d \mathbf{x}\right)^{\frac{1}{2}}\left(\int_{\Omega \times \partial B_{R}} d S(\mathbf{q}) d \mathbf{x}\right)^{\frac{1}{2}} \\
& \leq(\sigma(d)|\Omega|)^{\frac{1}{2}}\left(\int_{\Omega \times \partial B_{R}} R^{d-1+2 \delta}|\varphi|^{2} d S(\mathbf{q}) d \mathbf{x}\right)^{\frac{1}{2}} .
\end{aligned}
$$

From (3.1), (3.3), we observe that

$$
R^{d-1+2 \delta} \leq R^{d-1+2 \delta} Z^{\beta} \leq C R Z^{\beta} e^{\frac{\beta}{2}|R|^{2}} \leq C R M^{-\beta},
$$

where the constant $C$ depends on $\beta, d, \delta$. Thus, we obtain

$$
R^{\delta} \int_{\Omega \times \partial B_{R}}|\varphi| d S(\mathbf{q}) d \mathbf{x} \leq C\left(R \int_{\Omega \times \partial B_{R}} \frac{|\varphi|^{2}}{M^{\beta}} d S(\mathbf{q}) d \mathbf{x}\right)^{\frac{1}{2}},
$$

where $C$ is dependent on $\beta, d, \delta,|\Omega|$. Therefore, the desired result follows immediately from Lemma 3.2.

Recalling [2] and Lemma 3.1 in [1], we know that

$$
\begin{aligned}
& \mathcal{K}:=C^{\infty}\left(\bar{\Omega} ; C_{0}^{\infty}(D)\right) \text { is dense in } Z_{0}^{1}, \\
& \mathcal{K}^{0}:=C_{0}^{\infty}(\Omega \times D) \text { is dense in } Z_{0,0}^{1} .
\end{aligned}
$$

We can also show in a similar way that $\mathcal{K}$ is dense in $Y_{b}$ and $\mathcal{K}^{0}$ is dense in $Y_{b, 0}$. Next we introduce the positive and negative parts of a function $\varphi$ defined by

$$
[\varphi]_{+}:=\max \{\varphi, 0\}, \quad[\varphi]_{-}:=\min \{\varphi, 0\} .
$$

Lemma 3.4. Suppose Assumptions $\left(A_{1}\right)$ and $\left(A_{3}\right)$. If $\varphi \in Y_{b}^{\beta}$, then

$$
\begin{aligned}
& \nabla_{q}\left(\frac{[\varphi]_{+}}{M^{\beta}}\right)= \begin{cases}\nabla_{q}\left(\frac{\varphi}{M^{\beta}}\right), & \text { if } \varphi>0, \\
\mathbf{0}, & \text { if } \varphi \leq 0 ;\end{cases} \\
& \nabla_{q}\left(\frac{[\varphi]_{-}}{M^{\beta}}\right)= \begin{cases}\nabla_{q}\left(\frac{\varphi}{M^{\beta}}\right), & \text { if } \varphi<0, \\
\mathbf{0}, & \text { if } \varphi \geq 0 ;\end{cases} \\
& \nabla_{x}\left(\frac{[\varphi]_{+}}{M^{\beta}}\right)= \begin{cases}\nabla_{x}\left(\frac{\varphi}{M^{\beta}}\right), & \text { if } \varphi>0, \\
\mathbf{0}, & \text { if } \varphi \leq 0 ;\end{cases} \\
& \nabla_{x}\left(\frac{[\varphi]_{-}}{M^{\beta}}\right)= \begin{cases}\nabla_{x}\left(\frac{\varphi}{M^{\beta}}\right), & \text { if } \varphi<0, \\
\mathbf{0}, & \text { if } \varphi \geq 0 .\end{cases}
\end{aligned}
$$


Hence $[\varphi]_{+},[\varphi]_{-} \in Y_{b}^{\beta}$.

Proof. Suppose $\varphi \in Y_{b}^{\beta}$. For $\varepsilon>0$, introduce

$$
p_{\varepsilon}(s):=\left\{\begin{array}{l}
\left(s^{2}+\varepsilon^{2}\right)^{\frac{1}{2}}-\varepsilon \text { if } s \geq 0 \\
0 \text { if } s \leq 0
\end{array}\right.
$$

Then by Lemma 7.5 in [12], we have, for any $\eta \in C_{0}^{\infty}(\Omega \times D)$,

$$
\begin{aligned}
\int_{\Omega \times D} \frac{[\varphi]_{+}}{M^{\beta}} \nabla_{q} \eta d \mathbf{q} d \mathbf{x} & =\lim _{\varepsilon \rightarrow 0} \int_{\Omega \times D} p_{\varepsilon}\left(\frac{\varphi}{M^{\beta}}\right) \nabla_{q} \eta d \mathbf{q} d \mathbf{x} \\
& =-\lim _{\varepsilon \rightarrow 0} \int_{\varphi>0} \eta \frac{\varphi \nabla_{q} \frac{\varphi}{M^{\beta}}}{\left(\varphi^{2}+\varepsilon^{2} M^{2 \beta}\right)^{\frac{1}{2}}} d \mathbf{q} d \mathbf{x} \\
& =-\int_{\varphi>0} \eta \nabla_{q}\left(\frac{\varphi}{M^{\beta}}\right) d \mathbf{q} d \mathbf{x} .
\end{aligned}
$$

Therefore, the result is established for $\nabla_{q}\left(\frac{[\varphi]_{+}}{M^{\beta}}\right)$. The other results follow immediately.

Similar to the proof of Lemma 3.4, it follows that, if $\varphi \in Z_{b}^{\beta}$, then $[\varphi]_{+},[\varphi]_{-} \in Z_{b}^{\beta}$.

3.3. Mollifiers. In the equations (2.3)-(2.4) we have used isotropic Friedrichs mollifiers. It is not difficult to derive that $J_{\alpha}^{x}$ satisfies the following properties [2]:

$$
\begin{aligned}
\left\|J_{\alpha}^{x} \varphi\right\|_{L^{2}(\Omega)} & \leq\|\varphi\|_{L^{2}(\Omega)}, \quad \forall \varphi \in L^{2}(\Omega), \\
\left\|\left(I-J_{\alpha}^{x}\right) \varphi\right\|_{L^{2}(\Omega)} & \rightarrow 0 \text { as } \alpha \rightarrow 0, \quad \forall \varphi \in L^{2}(\Omega), \\
\int_{\Omega}\left(J_{\alpha}^{x} \varphi_{1}\right) \varphi_{2} d \mathbf{x} & =\int_{\Omega} \varphi_{1}\left(J_{\alpha}^{x} \varphi_{2}\right) d \mathbf{x}, \quad \forall \varphi_{1}, \varphi_{2} \in L^{2}(\Omega), \\
\frac{\partial}{\partial x_{i}}\left(J_{\alpha}^{x} \varphi\right) & =J_{\alpha}^{x}\left(\frac{\partial \varphi}{\partial x_{i}}\right), i=1 \rightarrow d, \quad \forall \varphi \in H_{0}^{1}(\Omega) .
\end{aligned}
$$

Thus, $\mathbf{J}_{\alpha}^{x}$ satisfies

$$
\begin{aligned}
\left\|\mathbf{J}_{\alpha}^{x} \mathbf{v}\right\|_{H^{1}(\Omega)} & \leq\|\mathbf{v}\|_{H^{1}(\Omega)}, \quad \forall \mathbf{v} \in \mathbf{H}_{0}^{1}(\Omega) \\
\left\|\mathbf{J}_{\alpha}^{x} \mathbf{v}\right\|_{W^{1, \infty}(\Omega)} & \leq C(\alpha)\|\mathbf{v}\|_{L^{1}(\Omega)}, \quad \forall \mathbf{v} \in \mathbf{L}^{1}(\Omega) .
\end{aligned}
$$

Moreover, we introduce a mollifier, the Helmholtz-Stokes mollifier $\mathbf{S}_{\gamma}$. This is motivated by Barrett et al. [1], Barrett and Süli [2] and Foias et al. [11].

Let $\mathbf{V}^{\prime}$ be the dual of $\mathbf{V},\langle\cdot, \cdot\rangle$ the duality between $\mathbf{V}^{\prime}$ and $\mathbf{V}$, and $\left\|\mathbf{S}_{\gamma} \cdot\right\|_{H^{1}(\Omega)}$ the norm on $V^{\prime}$. For given $\gamma \in(0,1]$ and $\mathbf{v} \in \mathbf{V}^{\prime}$, let $\mathbf{S}_{\gamma} \mathbf{v}$ be a smoothing of $\mathbf{v}$ defined as the unique solution of the Helmholtz-Stokes problem

$$
\int_{\Omega} \mathbf{S}_{\gamma} \mathbf{v} \cdot \mathbf{w} d \mathbf{x}+\gamma \int_{\Omega} \nabla_{x}\left(\mathbf{S}_{\gamma} \mathbf{v}\right): \nabla_{x} \mathbf{w} d \mathbf{x}=<\mathbf{v}, \mathbf{w}>, \quad \forall \mathbf{w} \in \mathbf{V}
$$

From $[1,2,13]$, we know that the Helmholtz-Stokes mollifier defined by (3.22) possesses the properties:

$$
\begin{aligned}
& <\mathbf{v}, \mathbf{S}_{\gamma} \mathbf{v}>=\int_{\Omega}\left[\gamma\left|\nabla_{x}\left(\mathbf{S}_{\gamma} \mathbf{v}\right)\right|^{2}+\left|\mathbf{S}_{\gamma} \mathbf{v}\right|^{2}\right] d \mathbf{x}, \quad \forall \mathbf{v} \in \mathbf{V}^{\prime} \\
& \left\|\mathbf{S}_{\gamma} \mathbf{v}\right\|_{L^{2}(\Omega)}^{2}+\gamma\left\|\nabla_{x}\left[\mathbf{S}_{\gamma} \mathbf{v}\right]\right\|_{L^{2}(\Omega)}^{2} \leq\|\mathbf{v}\|_{L^{2}(\Omega)}^{2}, \quad \forall \mathbf{v} \in \mathbf{L}^{2}(\Omega) \\
& \left\|\mathbf{S}_{\gamma} \mathbf{v}\right\|_{H^{1}(\Omega)}^{2} \leq C\|\mathbf{v}\|_{L^{2}(\Omega)}^{2} \leq C\left\|\nabla_{x} \mathbf{v}\right\|_{L^{2}(\Omega)}^{2}, \quad \forall \mathbf{v} \in \mathbf{V}, \\
& \left\|\left(\mathbf{I}-\mathbf{S}_{\gamma}\right) \mathbf{v}\right\|_{L^{2}(\Omega)}^{2}+\gamma\left\|\nabla_{x}\left(\mathbf{I}-\mathbf{S}_{\gamma}\right) \mathbf{v}\right\|_{L^{2}(\Omega)}^{2} \leq \gamma\left\|\nabla_{x} \mathbf{v}\right\|_{L^{2}(\Omega)}^{2}, \quad \forall \mathbf{v} \in \mathbf{V} .
\end{aligned}
$$


The Friedrichs mollifiers and the Helmholtz-Stokes mollifier have different roles in this paper. The former is to smooth some terms in the regularized Hookean dumbbell model, while the latter is just a technique in our estimates.

3.4. Some auxiliary assertions. Now we firstly show the property of $\mathbf{C}(\varphi)$ in Equation (2.3). Under the assumptions $\left(\mathrm{A}_{1}\right)$ and $\left(\mathrm{A}_{3}\right)$, we have $\forall \varphi \in X_{b}^{\beta}$,

$$
\begin{aligned}
\int_{\Omega}|\mathbf{C}(\varphi)|^{2} d \mathbf{x} & =\int_{\Omega} \sum_{i, j=1}^{d}\left(\int_{D} \varphi q_{i} q_{j} d \mathbf{q}\right)^{2} d \mathbf{x} \\
& \leq \int_{\Omega}\left(\sum_{i, j=1}^{d} \int_{D} q_{i}^{2} q_{j}^{2} M^{\beta} d \mathbf{q}\right)\left(\int_{D} \frac{|\varphi|^{2}}{M^{\beta}} d \mathbf{q}\right) d \mathbf{x} \\
& \leq\left(\int_{D} M^{\beta}|\mathbf{q}|^{4} d \mathbf{q}\right) \int_{\Omega \times D} \frac{|\varphi|^{2}}{M^{\beta}} d \mathbf{q} d \mathbf{x} .
\end{aligned}
$$

This together with (3.5) implies that

$$
\int_{\Omega}|\mathbf{C}(\varphi)|^{2} d \mathbf{x} \leq C(\beta) \int_{\Omega \times D} \frac{|\varphi|^{2}}{M^{\beta}} d \mathbf{q} d \mathbf{x} .
$$

We mention in particular that the constant $C(\beta)$ in (3.27) depends on the parameter $\beta$, and $C(\beta)$ converges to infinity as $\beta$ goes to zero. Next, we recall the Carlson inequality from Theorem 3.6 in [3], which is a useful tool in our investigation of $\mathbf{C}(\varphi)$.

LEMMA 3.5. Let $\eta$ be a real-valued measurable function on $\mathbb{R}_{+}^{d}$, and $A_{d}=\left\{\left\{b_{i}\right\}_{1}^{d}: b_{i}=\right.$ $\{0,1\}$ for $i=1,2, \ldots, d\}$. Then

$$
\int_{\mathbb{R}_{+}^{d}}|\eta(\mathbf{x})| d \mathbf{x} \leq(2 \pi)^{\frac{d}{2}}\left(\prod_{A_{d}} \int_{\mathbb{R}_{+}^{d}} \prod_{1}^{d} x_{i}^{2 b_{i}} \eta^{2}(\mathbf{x}) d \mathbf{x}\right)^{1 / 2^{d+1}}
$$

Note that (3.28) is also true in $\mathbb{R}^{d}$, just by a translation of variables. For convenience, we also recall the following lemmas from [30] and [5]:

Lemma 3.6. Let $X_{0}, X, X_{1}$ be Banach spaces such that $X_{0} \subset X \subset X_{1}$, where the injections are continuous and the $X_{i}$ are reflexive, $i=0,1$, and the injection $X_{0} \hookrightarrow X$ is compact. Let $\alpha_{i}>1, i=0,1$, then

$$
\left\{v \in L^{\alpha_{0}}\left(0, T ; X_{0}\right), \frac{\partial v}{\partial t} \in L^{\alpha_{1}}\left(0, T ; X_{1}\right)\right\} \hookrightarrow L^{\alpha_{0}}(0, T ; X)
$$

is compact.

Lemma 3.7. (Gagliardo-Nirenberg inequality) There exists a constant $C:=C(\Omega, r, d)$ such that for all $\eta \in H_{0}^{1}(\Omega)$,

$$
\|\eta\|_{L^{r}(\Omega)} \leq C\|\eta\|_{L^{2}(\Omega)}^{1-\gamma}\|\nabla \eta\|_{L^{2}(\Omega)}^{\gamma},
$$

where $r \in[2, \infty)$ if $d=2$, and $r \in[2,6]$ if $d=3$ and $\gamma=d\left(\frac{1}{2}-\frac{1}{r}\right)$. 


\section{The modified model and the Rothe method}

To obtain the global weak solutions in Definition 2.1, we now build a modified model.

It follows from (3.2) that (2.4) can be rewritten as

$$
\begin{aligned}
& \frac{\partial \psi_{\alpha, \varepsilon}}{\partial t}+\left(\mathbf{u}_{\alpha, \varepsilon} \cdot \nabla_{x}\right) \psi_{\alpha, \varepsilon}+\nabla_{q} \cdot\left(\nabla_{x}\left(\mathbf{J}_{\alpha}^{x} \mathbf{u}_{\alpha, \varepsilon}\right) \mathbf{q} \psi\right) \\
= & \frac{1}{2 \lambda} \nabla_{q} \cdot\left(M^{\beta} \nabla_{q} \frac{\psi_{\alpha, \varepsilon}}{M^{\beta}}+(1-\beta) \mathbf{q} \psi_{\alpha, \varepsilon}\right)+\varepsilon \Delta_{x} \psi_{\alpha, \varepsilon} \quad \text { in } \Omega \times D \times(0, T],
\end{aligned}
$$

and then (2.4) is equivalent to

$$
\begin{aligned}
& \frac{\partial\left(\left(1+|\mathbf{q}|^{b}\right) \psi_{\alpha, \varepsilon}\right)}{\partial t}+\left(\mathbf{u}_{\alpha, \varepsilon} \cdot \nabla_{x}\right)\left(\left(1+|\mathbf{q}|^{b}\right) \psi_{\alpha, \varepsilon}\right)+\left(1+|\mathbf{q}|^{b}\right) \nabla_{q} \cdot\left(\nabla_{x}\left(\mathbf{J}_{\alpha}^{x} \mathbf{u}_{\alpha, \varepsilon}\right) \mathbf{q} \psi\right) \\
= & \frac{1}{2 \lambda}\left(1+|\mathbf{q}|^{b}\right) \nabla_{q} \cdot\left(M^{\beta} \nabla_{q} \frac{\psi_{\alpha, \varepsilon}}{M^{\beta}}+(1-\beta) \mathbf{q} \psi_{\alpha, \varepsilon}\right)+\varepsilon \Delta_{x}\left(\left(1+|\mathbf{q}|^{b}\right) \psi_{\alpha, \varepsilon}\right) \\
& \text { in } \Omega \times D \times(0, T] .
\end{aligned}
$$

If we add the term $\frac{\beta}{2} A\left(\mathbf{u}_{\alpha, \varepsilon}\right)|\mathbf{q}|^{2}\left(1+|\mathbf{q}|^{b}\right) \frac{\psi_{\alpha, \varepsilon}}{M^{\beta}}$ into the left-hand side of (4.2), then we have a modification of (4.2), where $A(\mathbf{v}):=\left\|\nabla_{x}\left(\mathbf{J}_{\alpha}^{\mathbf{x}} \mathbf{v}\right)\right\|_{L^{\infty}(\Omega)} \forall \mathbf{v} \in \mathbf{L}^{1}(\Omega)$. Thus a modified model is derived composed of (2.1)-(2.3) and this modified equation for $\psi_{\alpha, \varepsilon}$ together with (2.8)-(2.11). Note that if $\beta=0$, this modified model is just the regularized Hookean dumbbell model. The solution of this modified model depends on four parameters $\alpha, \beta, \varepsilon, b$. For brevity, we introduce the notation in this section:

$$
\hat{\mathbf{u}}=\mathbf{u}_{\alpha, \beta, \varepsilon, b}, \hat{\psi}=\psi_{\alpha, \beta, \varepsilon, b} .
$$

The following result just gives the weak formulation of this modified model.

Proposition 4.1. Let assumptions $\left(A_{1}\right)-\left(A_{3}\right)$ be fulfilled. Then for any given $\alpha, \varepsilon \in$ $(0,1]$, there exists $(\hat{\mathbf{u}}, \hat{\psi})$ satisfying

$$
\begin{aligned}
& \hat{\mathbf{u}} \in L^{\infty}\left(0, T ; \mathbf{L}^{2}(\Omega)\right) \cap L^{2}(0, T ; \mathbf{V}) \cap W^{1, \frac{4}{d}}\left(0, T ; \mathbf{V}^{\prime}\right), \\
& \hat{\psi} \in L^{2}\left(0, T ; Z_{b}^{\beta,+}\right) \cap L^{\infty}\left(0, T ; L^{2}\left(\Omega \times D ;\left(1+|\mathbf{q}|^{b}\right) M^{-\beta}\right)\right), \\
& \mathbf{J}_{\alpha}^{x} \hat{\mathbf{u}} \in L^{\infty}\left(0, T ; \mathbf{W}^{1, \infty}(\Omega)\right), \mathbf{C}(\hat{\psi}) \in L^{\infty}\left(0, T ; \mathbf{L}^{2}(\Omega)\right), \\
& \int_{0}^{T}<\frac{\partial \hat{\mathbf{u}}}{\partial t}, \mathbf{w}>d t+\int_{0}^{T} \int_{\Omega}\left[\left[\left(\hat{\mathbf{u}} \cdot \nabla_{x}\right) \hat{\mathbf{u}}\right] \cdot \mathbf{w}+\nu \nabla_{x} \hat{\mathbf{u}}: \nabla_{x} \mathbf{w}\right] d \mathbf{x} d t \\
& =-\kappa \omega \int_{0}^{T} \int_{\Omega} \mathbf{C}(\hat{\psi}): \nabla_{x} \mathbf{w}_{\alpha} d \mathbf{x} d t, \quad \forall \mathbf{w} \in L^{\frac{4}{4-d}}(0, T ; \mathbf{V}), \\
& -\int_{0}^{T} \int_{\Omega \times D}\left(1+|\mathbf{q}|^{b}\right) \frac{\hat{\psi}}{M^{\beta}} \frac{\partial \varphi}{\partial t} d \mathbf{q} d \mathbf{x} d t-\int_{\Omega \times D}\left(1+|\mathbf{q}|^{b}\right) \frac{\psi_{0}(\cdot, \cdot)}{M^{\frac{\beta}{2}}} \varphi(\cdot, \cdot, 0) d \mathbf{q} d \mathbf{x} \\
& +\int_{0}^{T} \int_{\Omega \times D}\left[\frac{1}{2 \lambda} M^{\beta} \nabla_{q} \frac{\hat{\psi}}{M^{\beta}}-\left[\nabla_{x}\left(\mathbf{J}_{\alpha}^{x} \hat{\mathbf{u}}\right) \mathbf{q}\right] \hat{\psi}+\frac{1-\beta}{2 \lambda} \mathbf{q} \hat{\psi}\right] \\
& . \nabla_{q}\left(\left(1+|\mathbf{q}|^{b}\right) \frac{\varphi}{M^{\beta}}\right) d \mathbf{q} d \mathbf{x} d t+\frac{\beta}{2} \int_{0}^{T} \int_{\Omega \times D} A(\hat{\mathbf{u}})|\mathbf{q}|^{2}\left(1+|\mathbf{q}|^{b}\right) \frac{\hat{\psi}}{M^{\beta}} \varphi d \mathbf{q} d \mathbf{x} d t \\
& +\int_{0}^{T} \int_{\Omega \times D}\left(1+|\mathbf{q}|^{b}\right)\left[\varepsilon \nabla_{x} \hat{\psi}-\hat{\mathbf{u}} \hat{\psi}\right] \cdot \nabla_{x} \frac{\varphi}{M^{\beta}} d \mathbf{q} d \mathbf{x} d t=0, \forall \varphi \in \mathcal{Y}_{b}^{\beta}, \\
& \hat{\mathbf{u}}(\cdot, 0)=\mathbf{u}_{0}(\cdot),
\end{aligned}
$$


where $\mathcal{Y}_{b}^{\beta}$ be the completion of $C_{0}^{2}\left(-T, T ; Y_{b}^{\beta}\right)$ in the norm $\|\cdot\|_{\mathcal{Y}_{b}^{\beta}}$ defined by

$$
\begin{aligned}
\|\varphi\|_{\mathcal{Y}_{b}^{\beta}}:= & \|\varphi\|_{L^{1}\left(0, T ; Y_{b}^{\beta}\right)}+\left\|\left(1+|\mathbf{q}|^{b}\right)^{\frac{1}{2}} M^{\frac{\beta}{2}} \nabla_{x} \frac{\varphi}{M^{\beta}}\right\|_{L^{\frac{4}{4-d}}\left(0, T ; L^{2}(\Omega \times D)\right)} \\
& +\left\|\left(1+|\mathbf{q}|^{b}\right)^{\frac{1}{2}} M^{\frac{\beta}{2}} \nabla_{q} \frac{\varphi}{M^{\beta}}\right\|_{L^{2}\left(0, T ; L^{2}(\Omega \times D)\right)}+\left\||\mathbf{q}|^{\frac{b}{2}-1} \frac{\varphi}{M^{\frac{\beta}{2}}}\right\|_{L^{2}\left(0, T ; L^{2}(\Omega \times D)\right)} \\
& +\left\|\left(1+|\mathbf{q}|^{b}\right)^{\frac{1}{2}} M^{-\frac{\beta}{2}} \frac{\partial \varphi}{\partial t}\right\|_{L^{1}\left(0, T ; L^{2}(\Omega \times D)\right)} .
\end{aligned}
$$

We will adapt the Rothe method to prove Proposition 4.1. The Rothe method is frequently used to prove the solvability of evolution problems (see, e.g., $[16,26]$ ). Its principle consists in semidiscretization with respect to the time variable, and the original problem is approximated by a sequence of Rothe approximation functions. Now we proceed as follows.

4.1. Solvability of time-discretized problems. In order to solve the modified problem by the Rothe method, we subdivide the interval $[0, T]$ into $N$ units of length $\Delta t(T=N \Delta t)$. Set $t_{n}=n \Delta t, n=0, \ldots, N$. For given $\alpha, \varepsilon \in(0,1], b$ and $\beta$ satisfying Assumption $\left(\mathrm{A}_{1}\right)$ and $\left(\mathrm{A}_{3}\right)$ respectively, we define the sequence of $\left\{\hat{\mathbf{u}}^{n}, \hat{\psi}^{n}\right\}_{n=0}^{N}$ by an implicit scheme as follows:

$$
\begin{aligned}
& \hat{\mathbf{u}}^{0}=\mathbf{S}_{\Delta t} \mathbf{u}_{0}, \quad \hat{\psi}^{0}=\frac{M^{\frac{\beta}{2}}}{G_{c}} \psi_{0}, \\
& \int_{\Omega} \frac{\hat{\mathbf{u}}^{n}-\hat{\mathbf{u}}^{n-1}}{\Delta t} \cdot \mathbf{w} d \mathbf{x}+\int_{\Omega}\left[\left(\hat{\mathbf{u}}^{n-1} \cdot \nabla_{x}\right) \hat{\mathbf{u}}^{n}\right] \cdot \mathbf{w} d \mathbf{x}+\nu \int_{\Omega} \nabla_{x} \hat{\mathbf{u}}^{n}: \nabla_{x} \mathbf{w} d \mathbf{x} \\
& =-\kappa \omega \int_{\Omega} \mathbf{C}\left(\hat{\psi}^{n}\right): \nabla_{x}\left(\mathbf{w}_{\alpha}\right) d \mathbf{x}, \quad \forall \mathbf{w} \in \mathbf{V}, \\
& \int_{\Omega \times D}\left(1+|\mathbf{q}|^{b}\right) G_{c} \frac{\hat{\psi}^{n}-\hat{\psi}^{n-1}}{\Delta t} \frac{\varphi}{M^{\beta}} d \mathbf{q} d \mathbf{x} \\
& \quad+\frac{b}{2} \int_{\Omega \times D}|\mathbf{q}|^{b}\left[A\left(\hat{\mathbf{u}}^{n}\right) \hat{\psi}^{n}-A\left(\hat{\mathbf{u}}^{n-1}\right) \hat{\psi}^{n-1}\right] \frac{\varphi}{M^{\beta}} d \mathbf{q} d \mathbf{x} \\
& \quad+\int_{\Omega \times D}\left[\frac{W_{c}}{2 \lambda} M^{\beta} \nabla_{q} \frac{\hat{\psi}^{n}}{M^{\beta}}+\frac{1-\beta}{2 \lambda} \mathbf{q} \hat{\psi}^{n}-\left[\nabla_{x}\left(\mathbf{J}_{\alpha}^{x} \hat{\mathbf{u}}^{n}\right) \mathbf{q}\right] \hat{\psi}^{n}\right] \\
& \quad \nabla_{q}\left(\left(1+|\mathbf{q}|^{b}\right) \frac{\varphi}{M^{\beta}}\right) d \mathbf{q} d \mathbf{x}+\frac{\beta}{2} \int_{\Omega \times D} A\left(\hat{\mathbf{u}}^{n}\right)|\mathbf{q}|^{2}\left(1+|\mathbf{q}|^{b}\right) \frac{\hat{\psi}^{n} \varphi}{M^{\beta}} d \mathbf{q} d \mathbf{x} \\
& \quad+\int_{\Omega \times D}\left(1+|\mathbf{q}|^{b}\right)\left(\varepsilon \nabla_{x} \hat{\psi}^{n}-\hat{\mathbf{u}}^{n} \hat{\psi}^{n}\right) \cdot \nabla_{x} \frac{\varphi}{M^{\beta}} d \mathbf{q} d \mathbf{x}=0, \quad \forall \varphi \in Y_{b}^{\beta},
\end{aligned}
$$

where

$$
G_{c}:=1+\triangle t|\mathbf{q}|^{4}, W_{c}:=1+\triangle t|\mathbf{q}|^{2} .
$$

Then from (4.10) and Assumption $\left(\mathrm{A}_{2}\right)$ we know that $\hat{\mathbf{u}}^{0} \in \mathbf{V}, \hat{\psi}^{0} \geq 0$ a.e. in $\Omega \times D$. Further, by using (3.4), (3.24) and (4.13) we have

$$
\begin{aligned}
\int_{\Omega}\left[\left|\hat{\mathbf{u}}^{0}\right|^{2}\right. & \left.+\Delta t\left|\nabla_{x} \hat{\mathbf{u}}^{0}\right|^{2}\right] d \mathbf{x}+\int_{\Omega \times D}\left[G_{c}(1+U) \hat{\psi}^{0}+\left(1+|\mathbf{q}|^{b}\right) G_{c} \frac{\left|\hat{\psi}^{0}\right|^{2}}{M^{\beta}}\right] d \mathbf{q} d \mathbf{x} \\
& \leq C .
\end{aligned}
$$


In addition, $\hat{\psi}^{0} \rightarrow \psi_{0}$ in $L^{2}(\Omega \times D)$ as $(\triangle t, \beta) \rightarrow\left(0_{+}, 0_{+}\right)$. Noticing that $\mathcal{V}$ is dense in $H$, it follows from (3.26) that $\hat{\mathbf{u}}^{0} \rightarrow \mathbf{u}_{0}$ in $\mathbf{H}$ as $\triangle t \rightarrow 0_{+}$.

REMARK 4.2. 1) (4.10)-(4.12) is just the time-discretized formulation of the modified problem (in weak formulation). In this discretized formulation, we insert $G_{c}, W_{c}$ and the term

$$
\frac{b}{2} \int_{\Omega \times D}|\mathbf{q}|^{b}\left[A\left(\hat{\mathbf{u}}^{n}\right) \hat{\psi}^{n}-A\left(\hat{\mathbf{u}}^{n-1}\right) \hat{\psi}^{n-1}\right] \frac{\varphi}{M^{\beta}} d \mathbf{q} d \mathbf{x}
$$

into (4.12). Our goal is to use trace results (3.14) and (3.15) to verify the coerciveness of a bilinear functional (4.18) below and derive a priori estimates for the time-discretized functions.

2) This discretized formulation leads to a set of nonlinearly coupled elliptic boundary value problems to determine $\left(\hat{\mathbf{u}}^{n}, \hat{\psi}^{n}\right)$. The existence of these solutions is a consequence of the Lax-Milgram theorem [12] and the Schauder fixed point theorem [12].

It is obvious that (4.11)-(4.12) can be rewritten in the variational forms:

$$
\begin{aligned}
& b\left(\hat{\mathbf{u}}^{n-1}\right)\left(\hat{\mathbf{u}}^{n}, \mathbf{w}\right)=\int_{\Omega}\left[\hat{\mathbf{u}}^{n-1} \cdot \mathbf{w}-\Delta t \kappa \omega \mathbf{C}\left(\hat{\psi}^{n}\right): \nabla_{x}\left(\mathbf{w}_{\alpha}\right)\right] d \mathbf{x}, \quad \forall \mathbf{w} \in \mathbf{V}, \\
& a\left(\hat{\mathbf{u}}^{n}\right)\left(\hat{\psi}^{n}, \varphi\right)=\int_{\Omega \times D}\left[\left(1+|\mathbf{q}|^{b}\right) G_{c}+\triangle t \frac{b}{2}|\mathbf{q}|^{b} A\left(\hat{\mathbf{u}}^{n-1}\right)\right] \frac{\hat{\psi}^{n-1} \varphi}{M^{\beta}} d \mathbf{q} d \mathbf{x}, \forall \varphi \in Y_{b}^{\beta} .
\end{aligned}
$$

Here,

$$
\begin{gathered}
b\left(\hat{\mathbf{u}}^{n-1}\right)\left(\mathbf{w}_{1}, \mathbf{w}_{2}\right):=\int_{\Omega}\left[\mathbf{w}_{1}+\Delta t\left(\hat{\mathbf{u}}^{n-1} \cdot \nabla_{x}\right) \mathbf{w}_{1}\right] \cdot \mathbf{w}_{2} d \mathbf{x}+\Delta t \nu \int_{\Omega} \nabla_{x} \mathbf{w}_{1}: \nabla_{x} \mathbf{w}_{2} d \mathbf{x} \\
\forall \mathbf{w}_{1}, \mathbf{w}_{2} \in \mathbf{V} \\
a(\mathbf{v})\left(\varphi_{1}, \varphi_{2}\right):=\int_{\Omega \times D}\left(\left[\left(1+|\mathbf{q}|^{b}\right)\left(G_{c}+\Delta t \frac{\beta}{2} A(\mathbf{v})|\mathbf{q}|^{2}\right)+\Delta t \frac{b}{2}|\mathbf{q}|^{b} A(\mathbf{v})\right] \frac{\varphi_{1} \varphi_{2}}{M^{\beta}}\right. \\
+\Delta t\left[\frac{W_{c}}{2 \lambda} M^{\beta} \nabla_{q} \frac{\varphi_{1}}{M^{\beta}}+\frac{1-\beta}{2 \lambda} \mathbf{q} \varphi_{1}-\nabla_{x}\left(\mathbf{v}_{\alpha}\right) \mathbf{q} \varphi_{1}\right] \cdot \nabla_{q}\left(\left(1+|\mathbf{q}|^{b}\right) \frac{\varphi_{2}}{M^{\beta}}\right) \\
\left.+\Delta t\left(1+|\mathbf{q}|^{b}\right)\left[\varepsilon \nabla_{x} \varphi_{1}-\mathbf{v} \varphi_{1}\right] \cdot \nabla_{x} \frac{\varphi_{2}}{M^{\beta}}\right) d \mathbf{q} d \mathbf{x}, \\
\forall \varphi_{1}, \varphi_{2} \in Y_{b}^{\beta}, \forall \mathbf{v} \in \mathbf{Y}^{r},
\end{gathered}
$$

and for $r>d$,

$$
\begin{aligned}
\mathbf{Y}^{r}:= & \left\{\mathbf{v} \in \mathbf{L}^{r}(\Omega): \int_{\Omega} \mathbf{v} \varphi_{1} \cdot \nabla_{x} \varphi_{2} d \mathbf{x}=-\int_{\Omega} \mathbf{v} \varphi_{2} \cdot \nabla_{x} \varphi_{1} d \mathbf{x},\right. \\
& \left.\int_{\Omega} \varphi_{3} \nabla_{x} \cdot\left(\mathbf{v}_{\alpha}\right)=0, \forall \varphi_{1}, \varphi_{2} \in H^{1}(\Omega), \forall \varphi_{3} \in L^{1}(\Omega)\right\} .
\end{aligned}
$$

We can see that $b\left(\hat{\mathbf{u}}^{n-1}\right)(\cdot, \cdot)$ is a continuous and coercive bilinear functional on $\mathbf{V} \times \mathbf{V}$ by using the fact

$$
\int_{\Omega}\left[\left(\mathbf{v} \cdot \nabla_{x}\right) \mathbf{w}_{1}\right] \cdot \mathbf{w}_{2} d \mathbf{x}=-\int_{\Omega}\left[\left(\mathbf{v} \cdot \nabla_{x}\right) \mathbf{w}_{2}\right] \cdot \mathbf{w}_{1} d \mathbf{x}, \quad \forall \mathbf{v} \in \mathbf{V}, \forall \mathbf{w}_{1}, \mathbf{w}_{2} \in \mathbf{H}_{0}^{1}(\Omega) .
$$

Moreover, on noting (3.21), (3.7) and (4.19), we deduce from (4.18) that $a(\mathbf{v})(\cdot, \cdot)$ is a continuous bilinear functional on $Y_{b}^{\beta} \times Y_{b}^{\beta}$. In the following we will show that it 
is also coercive. By (3.7) and (4.19), we know that

$$
\int_{\Omega \times D} \mathbf{v} \varphi \cdot \nabla_{x} \frac{\varphi}{M^{\beta}} d \mathbf{q} d \mathbf{x}=0, \quad \forall \mathbf{v} \in \mathbf{Y}^{r}, \forall \varphi \in Y_{b}^{\beta} .
$$

Thus,

$$
\begin{aligned}
& a(\mathbf{v})(\varphi, \varphi) \\
& =\int_{\Omega \times D}\left[\left(1+|\mathbf{q}|^{b}\right) G_{c}+\Delta t \frac{b}{2}|\mathbf{q}|^{b} A(\mathbf{v})\right] \frac{|\varphi|^{2}}{M^{\beta}} d \mathbf{q} d \mathbf{x} \\
& +\Delta t \int_{\Omega \times D}\left[\frac{W_{c}}{2 \lambda} M^{\beta} \nabla_{q} \frac{\varphi}{M^{\beta}}+\frac{1-\beta}{2 \lambda} \mathbf{q} \varphi-\nabla_{x}\left(\mathbf{v}_{\alpha}\right) \mathbf{q} \varphi\right] \cdot \nabla_{q}\left(\left(1+|\mathbf{q}|^{b}\right) \frac{\varphi}{M^{\beta}}\right) d \mathbf{q} d \mathbf{x} \\
& +\varepsilon \Delta t \int_{\Omega \times D}\left(1+|\mathbf{q}|^{b}\right) M^{\beta}\left|\nabla_{x} \frac{\varphi}{M^{\beta}}\right|^{2} d \mathbf{q} d \mathbf{x}+\frac{\beta}{2} \Delta t \int_{\Omega \times D} A(\mathbf{v})|\mathbf{q}|^{2}\left(1+|\mathbf{q}|^{b}\right) \frac{|\varphi|^{2}}{M^{\beta}} d \mathbf{q} d \mathbf{x}, \\
& \forall \mathbf{v} \in \mathbf{Y}^{r}, \forall \varphi \in Y_{b}^{\beta} .
\end{aligned}
$$

By using (3.2), (4.13) and Lemma 3.2, we treat the second part of the right-hand side of (4.21) term by term as follows.

$$
\begin{aligned}
& \int_{\Omega \times D} \mathbf{q} \varphi \cdot \nabla_{q}\left(\left(1+|\mathbf{q}|^{b}\right) \frac{\varphi}{M^{\beta}}\right) d \mathbf{q} d \mathbf{x} \\
&= b \int_{\Omega \times D}|\mathbf{q}|^{b} \frac{|\varphi|^{2}}{M^{\beta}} d \mathbf{q} d \mathbf{x}+\frac{1}{2} \int_{\Omega \times D}\left(1+|\mathbf{q}|^{b}\right) \mathbf{q} M^{\beta} \cdot \nabla_{q}\left(\frac{\varphi}{M^{\beta}}\right)^{2} d \mathbf{q} d \mathbf{x} \\
&= \frac{b}{2} \int_{\Omega \times D}|\mathbf{q}|^{b} \frac{|\varphi|^{2}}{M^{\beta}} d \mathbf{q} d \mathbf{x}-\frac{d}{2} \int_{\Omega \times D}\left(1+|\mathbf{q}|^{b}\right) \frac{|\varphi|^{2}}{M^{\beta}} d \mathbf{q} d \mathbf{x}+\frac{\beta}{2} \int_{\Omega \times D}\left(1+|\mathbf{q}|^{b}\right)|\mathbf{q}|^{2} \frac{|\varphi|^{2}}{M^{\beta}} d \mathbf{q} d \mathbf{x} \\
& \Delta t \int_{\Omega \times D}\left[\nabla_{x}\left(\mathbf{v}_{\alpha}\right) \mathbf{q} \varphi\right] \cdot \nabla_{q}\left(\left(1+|\mathbf{q}|^{b}\right) \frac{\varphi}{M^{\beta}}\right) d \mathbf{q} d \mathbf{x} \\
&= b \Delta t \int_{\Omega \times D}\left[\mathbf{q}^{T} \nabla_{x}\left(\mathbf{v}_{\alpha}\right) \mathbf{q}\right]|\mathbf{q}|^{b-2} \frac{|\varphi|^{2}}{M^{\beta}} d \mathbf{q} d \mathbf{x}+\frac{\Delta t}{2} \int_{\Omega \times D}\left(1+|\mathbf{q}|^{b}\right)\left[\nabla_{x}\left(\mathbf{v}_{\alpha}\right) \mathbf{q} M^{\beta}\right] \\
&= \frac{b}{2} \Delta t \int_{\Omega \times D}\left[\mathbf{q}^{T} \nabla_{x}\left(\mathbf{v}_{\alpha}\right) \mathbf{q}\right]|\mathbf{q}|^{b-2} \frac{|\varphi|^{2}}{M^{\beta}} d \mathbf{q} d \mathbf{x} \\
& \quad+\frac{\beta}{2} \Delta t \int_{\Omega \times D}\left(1+|\mathbf{q}|^{b}\right)\left[\mathbf{q}^{T} \nabla_{x}\left(\mathbf{v}_{\alpha}\right) \mathbf{q}\right] \frac{|\varphi|^{2}}{M^{\beta}} d \mathbf{q} d \mathbf{x} \\
& \leq \frac{b}{2} \Delta t \int_{\Omega \times D} A(\mathbf{v})|\mathbf{q}|^{b} \frac{|\varphi|^{2}}{M^{\beta}} d \mathbf{q} d \mathbf{x}+\frac{\beta}{2} \Delta t \int_{\Omega \times D}\left(1+|\mathbf{q}|^{b}\right)|\mathbf{q}|^{2} A(\mathbf{v}) \frac{|\varphi|^{2}}{M^{\beta}} d \mathbf{q} d \mathbf{x}, \quad \text { (4.23) }
\end{aligned}
$$

and

$$
\begin{aligned}
& \int_{\Omega \times D} W_{c} M^{\beta} \nabla_{q} \frac{\varphi}{M^{\beta}} \cdot \nabla_{q}\left(\left(1+|\mathbf{q}|^{b}\right) \frac{\varphi}{M^{\beta}}\right) d \mathbf{q} d \mathbf{x} \\
= & \frac{b}{2} \int_{\Omega \times D} W_{c} M^{\beta}|\mathbf{q}|^{b-2} \nabla_{q}\left(\frac{\varphi}{M^{\beta}}\right)^{2} \cdot \mathbf{q} d \mathbf{q} d \mathbf{x}+\int_{\Omega \times D} W_{c} M^{\beta}\left|\nabla_{q} \frac{\varphi}{M^{\beta}}\right|^{2}\left(1+|\mathbf{q}|^{b}\right) d \mathbf{q} d \mathbf{x} \\
= & -\frac{b(b-2+d)}{2} \int_{\Omega \times D} W_{c}|\mathbf{q}|^{b-2} \frac{|\varphi|^{2}}{M^{\beta}} d \mathbf{q} d \mathbf{x}+\int_{\Omega \times D} W_{c} M^{\beta}\left|\nabla_{q} \frac{\varphi}{M^{\beta}}\right|^{2}\left(1+|\mathbf{q}|^{b}\right) d \mathbf{q} d \mathbf{x} \\
& -b \triangle t \int_{\Omega \times D}|\mathbf{q}|^{b} \frac{|\varphi|^{2}}{M^{\beta}} d \mathbf{q} d \mathbf{x}+\frac{b \beta}{2} \int_{\Omega \times D} W_{c}|\mathbf{q}|^{b} \frac{|\varphi|^{2}}{M^{\beta}} d \mathbf{q} d \mathbf{x} .
\end{aligned}
$$


Utilization of the above relations in (4.21) yields

$$
\begin{aligned}
& a(\mathbf{v})(\varphi, \varphi) \\
& \geq \int_{\Omega \times D}\left(\left(1+|\mathbf{q}|^{b}\right) G_{c} \frac{|\varphi|^{2}}{M^{\beta}}+\Delta t\left(1+|\mathbf{q}|^{b}\right)\left(\frac{W_{c}}{2 \lambda} M^{\beta}\left|\nabla_{q} \frac{\varphi}{M^{\beta}}\right|^{2}+\varepsilon M^{\beta}\left|\nabla_{x} \frac{\varphi}{M^{\beta}}\right|^{2}\right)\right) d \mathbf{q} d \mathbf{x} \\
& \quad+\frac{b(1-\beta)}{4 \lambda} \Delta t \int_{\Omega \times D}|\mathbf{q}|^{b} \frac{|\varphi|^{2}}{M^{\beta}} d \mathbf{q} d \mathbf{x}+\frac{b \beta \Delta t}{4 \lambda} \int_{\Omega \times D} W_{c}|\mathbf{q}|^{b} \frac{|\varphi|^{2}}{M^{\beta}} d \mathbf{q} d \mathbf{x} \\
& +\frac{\beta(1-\beta)}{4 \lambda} \Delta t \int_{\Omega \times D}\left(1+|\mathbf{q}|^{b}\right)|\mathbf{q}|^{2} \frac{|\varphi|^{2}}{M^{\beta}} d \mathbf{q} d \mathbf{x}-\frac{d(1-\beta)}{4 \lambda} \Delta t \int_{\Omega \times D}\left(1+|\mathbf{q}|^{b}\right) \frac{|\varphi|^{2}}{M^{\beta}} d \mathbf{q} d \mathbf{x} \\
& \quad-\frac{b}{2 \lambda}(\triangle t)^{2} \int_{\Omega \times D}|\mathbf{q}|^{b} \frac{|\varphi|^{2}}{M^{\beta}} d \mathbf{q} d \mathbf{x}-\frac{b(b-2+d)}{4 \lambda} \Delta t \int_{\Omega \times D} W_{c}|\mathbf{q}|^{b-2} \frac{|\varphi|^{2}}{M^{\beta}} d \mathbf{q} d \mathbf{x}, \\
& \forall \mathbf{v} \in \mathbf{Y}^{r}, \forall \varphi \in Y_{b}^{\beta} .
\end{aligned}
$$

Here the last term on the right-hand side of (4.21) is eliminated by the second term on the right-hand side of (4.23). This is why we add the term $\frac{\beta}{2} A\left(\mathbf{u}_{\alpha, \varepsilon}\right)|\mathbf{q}|^{2}\left(1+|\mathbf{q}|^{b}\right) \frac{\psi_{\alpha, \varepsilon}}{M^{\beta}}$ in the modified model; see (4.8). Here we can also see the remarkable role of the parameter $\beta$.

Now let $\triangle t$ be bounded by a constant, say, $\triangle t \leq 1$. Then

$$
\begin{aligned}
& -\frac{d(1-\beta)}{4 \lambda} \Delta t \int_{\Omega \times D}\left(1+|\mathbf{q}|^{b}\right) \frac{|\varphi|^{2}}{M^{\beta}} d \mathbf{q} d \mathbf{x}-\frac{b}{2 \lambda}(\triangle t)^{2} \int_{\Omega \times D}|\mathbf{q}|^{b} \frac{|\varphi|^{2}}{M^{\beta}} d \mathbf{q} d \mathbf{x} \\
& -\frac{b(b-2+d)}{4 \lambda} \triangle t \int_{\Omega \times D} W_{c}|\mathbf{q}|^{b-2} \frac{|\varphi|^{2}}{M^{\beta}} d \mathbf{q} d \mathbf{x} \\
\geq & -\frac{2 b(b-1+d)+d}{4 \lambda} \triangle t \int_{\Omega \times D} G_{c}\left(1+|\mathbf{q}|^{b}\right) \frac{|\varphi|^{2}}{M^{\beta}} d \mathbf{q} d \mathbf{x} .
\end{aligned}
$$

Thus,

$$
\begin{aligned}
a(\mathbf{v})(\varphi, \varphi) \geq & \left(1-\frac{2 b(b-1+d)+d}{4 \lambda} \triangle t\right) \int_{\Omega \times D}\left(1+|\mathbf{q}|^{b}\right) G_{c} \frac{|\varphi|^{2}}{M^{\beta}} d \mathbf{q} d \mathbf{x} \\
& +\Delta t \int_{\Omega \times D}\left(1+|\mathbf{q}|^{b}\right)\left(\frac{W_{c}}{2 \lambda} M^{\beta}\left|\nabla_{q} \frac{\varphi}{M^{\beta}}\right|^{2}+\varepsilon M^{\beta}\left|\nabla_{x} \frac{\varphi}{M^{\beta}}\right|^{2}\right) d \mathbf{q} d \mathbf{x} \\
& +\frac{b(1-\beta)}{4 \lambda} \Delta t \int_{\Omega \times D}|\mathbf{q}|^{b} \frac{|\varphi|^{2}}{M^{\beta}} d \mathbf{q} d \mathbf{x}+\frac{b \beta \triangle t}{4 \lambda} \int_{\Omega \times D} W_{c}|\mathbf{q}|^{b} \frac{|\varphi|^{2}}{M^{\beta}} d \mathbf{q} d \mathbf{x} \\
& +\frac{\beta(1-\beta)}{4 \lambda} \Delta t \int_{\Omega \times D}\left(1+|\mathbf{q}|^{b}\right)|\mathbf{q}|^{2} \frac{|\varphi|^{2}}{M^{\beta}} d \mathbf{q} d \mathbf{x}, \quad \forall \mathbf{v} \in \mathbf{Y}^{r}, \forall \varphi \in Y_{b}^{\beta} .(4.2
\end{aligned}
$$

Let $\triangle t<\min \left(1, \frac{4 \lambda}{2 b(b-1+d)+d}\right)$. Then we can see from $(4.24)$ that $a(\mathbf{v})(\cdot, \cdot)$ is coercive on $Y_{b}^{\beta} \times Y_{b}^{\beta}$.

Therefore, for given $\hat{\mathbf{v}} \in \mathbf{Y}^{r}(r>d)$, by the Lax-Milgram theorem, we can uniquely determine $\{\tilde{\phi}, \tilde{\mathbf{v}}\} \in Y_{b}^{\beta} \times \mathbf{V}$ by

$$
\begin{aligned}
a(\hat{\mathbf{v}})(\tilde{\phi}, \varphi) & =\int_{\Omega \times D}\left[\left(1+|\mathbf{q}|^{b}\right) G_{c}+\Delta t \frac{b}{2}|\mathbf{q}|^{b} A\left(\hat{\mathbf{u}}^{n-1}\right)\right] \\
& \cdot \frac{\hat{\psi}^{n-1} \varphi}{M^{\beta}} d \mathbf{q} d \mathbf{x}, \forall \varphi \in Y_{b}^{\beta}, \\
b\left(\hat{\mathbf{u}}^{n-1}\right)(\tilde{\mathbf{v}}, \mathbf{w})= & \int_{\Omega}\left[\hat{\mathbf{u}}^{n-1} \cdot \mathbf{w}-\Delta t \kappa \omega \mathbf{C}(\tilde{\phi}): \nabla_{x}\left(\mathbf{w}_{\alpha}\right)\right] d \mathbf{x}, \quad \forall \mathbf{w} \in \mathbf{V} .
\end{aligned}
$$


This completely defines the map $\mathbf{G}: \mathbf{Y}^{r}(r>d) \rightarrow \mathbf{V} \subset \mathbf{Y}^{r}$, which takes $\hat{\mathbf{v}}$ to $\tilde{\mathbf{v}}=\mathbf{G}(\hat{\mathbf{v}})$ via (4.25)-(4.26). The following lemma gives the property of $\mathbf{G}$.

LEMma 4.1. Let $\left(\hat{\mathrm{u}}^{n-1}, \hat{\psi}^{n-1}\right) \in \mathbf{V} \times L^{2,+}\left(\Omega \times D ;\left(1+|\mathbf{q}|^{b}\right) M^{-\beta} G_{c}\right)$. If $\triangle t$ is small enough, then $\mathbf{G}$ has a fixed point in $\mathbf{Y}^{r}, r \in(d, 6)$.

This can be proved by energy estimates and the Schauder fixed point theorem. The proof is given in the Appendix for self-containedness.

By Lemma 4.1, there exists a solution $\left(\hat{\mathbf{u}}^{n}, \hat{\psi}^{n}\right) \in \mathbf{V} \times Y_{b}^{\beta}$ to (4.11)-(4.12). Moreover, from Lemma 3.4 and $\hat{\psi}^{n} \in Y_{b}^{\beta}$, we know that $\left[\hat{\psi}^{n}\right]_{-} \in Y_{b}^{\beta}$. It follows from (4.16), (4.18) and $\hat{\psi}^{n-1} \in L^{2,+}\left(\Omega \times D ;\left(1+|\mathbf{q}|^{b}\right) M^{-\beta} G_{c}\right)$ that

$$
\begin{aligned}
& a\left(\hat{\mathbf{u}}^{n}\right)\left(\left[\hat{\psi}^{n}\right]_{-},\left[\hat{\psi}^{n}\right]_{-}\right)=a\left(\hat{\mathbf{u}}^{n}\right)\left(\hat{\psi}^{n},\left[\hat{\psi}^{n}\right]_{-}\right) \\
& =\int_{\Omega \times D}\left[\left(1+|\mathbf{q}|^{b}\right) G_{c}+\triangle t \frac{b}{2}|\mathbf{q}|^{b} A\left(\hat{\mathbf{u}}^{n-1}\right)\right] \frac{\hat{\psi}^{n-1}\left[\hat{\psi}^{n}\right]_{-}}{M^{\beta}} d \mathbf{q} d \mathbf{x} \leq 0 .
\end{aligned}
$$

Then, the coerciveness of $a\left(\hat{\mathbf{u}}^{n}\right)(\cdot, \cdot)$ in $Y_{b}^{\beta} \times Y_{b}^{\beta}$ yields that $\left[\hat{\psi}^{n}\right]_{-}=0$. Thus, $\hat{\psi}^{n} \in Y_{b}^{\beta,+}$.

Therefore, when $\left(\hat{\mathrm{u}}^{n-1}, \hat{\psi}^{n-1}\right) \in \mathbf{V} \times L^{2,+}\left(\Omega \times D ;\left(1+|\mathbf{q}|^{b}\right) M^{-\beta} G_{c}\right)$, Lemma 4.1 implies the existence of a solution $\left(\hat{\mathrm{u}}^{n}, \hat{\psi}^{n}\right) \in \mathbf{V} \times Y_{b}^{\beta,+}$ to (4.11)-(4.12). Then starting with $n=0$, this iterative procedure yields a sequence $\left\{\hat{\mathbf{u}}^{n}, \hat{\psi}^{n}\right\}_{n=1}^{N}$ of (4.11)-(4.12) that is well defined in $\mathbf{V} \times Y_{b}^{\beta,+}$.

4.2. A priori estimates for the time-discretized problems. In this subsection, we will devote ourselves to a series of a priori estimates independent of $n$ for the approximate functions $\hat{\mathbf{u}}^{n}$ and $\hat{\psi}^{n}$. Now we firstly state a discrete Gronwall inequality.

LEMma 4.2. Let $\left\{A_{n}\right\},\left\{B_{n}\right\}$ be nonnegative sequences satisfying

$$
A_{n}+B_{n} \leq(1+C \Delta t) A_{n-1}, \quad \forall n \geq 1 .
$$

Then we have

$$
\max _{n=0 \rightarrow N} A_{n}+\sum_{n=0}^{N} B_{n} \leq C .
$$

Note that $\frac{M^{\beta}}{1+|\mathbf{q}|^{6}} \in Y_{b}^{\beta}$, under the assumptions $\left(\mathrm{A}_{1}\right)$ and $\left(\mathrm{A}_{3}\right)$. Then by choosing $\varphi=\frac{M^{\beta}}{1+|\mathbf{q}|^{b}}$ in (4.16), we have

$$
\begin{aligned}
\int_{\Omega \times D} & G_{c}\left(\hat{\psi}^{n}-\hat{\psi}^{n-1}\right) d \mathbf{q} d \mathbf{x}+\frac{\beta}{2} \triangle t \int_{\Omega \times D} A\left(\hat{\mathbf{u}}^{n}\right)|\mathbf{q}|^{2} \hat{\psi}^{n} d \mathbf{q} d \mathbf{x} \\
& +\frac{b}{2} \triangle t \int_{\Omega \times D} \frac{|\mathbf{q}|^{b}}{1+|\mathbf{q}|^{b}}\left(A\left(\hat{\mathbf{u}}^{n}\right) \hat{\psi}^{n}-A\left(\hat{\mathbf{u}}^{n-1}\right) \hat{\psi}^{n-1}\right) d \mathbf{q} d \mathbf{x}=0 .
\end{aligned}
$$

Summing up the above identity for all $n$ and noticing (3.21) and (4.14), we have

$$
\begin{aligned}
& \max _{n=0 \rightarrow N}\left[\int_{\Omega \times D} G_{c} \hat{\psi}^{n} d \mathbf{q} d \mathbf{x}\right]+\frac{b}{2} \max _{n=0 \rightarrow N}\left[\triangle t \int_{\Omega \times D} \frac{|\mathbf{q}|^{b}}{1+|\mathbf{q}|^{b}} A\left(\hat{\mathbf{u}}^{n}\right) \hat{\psi}^{n} d \mathbf{q} d \mathbf{x}\right] \\
\leq & 2 \int_{\Omega \times D} G_{c} \hat{\psi}^{0} d \mathbf{q} d \mathbf{x}+b \triangle t A\left(\hat{\mathbf{u}}^{0}\right) \int_{\Omega \times D} \frac{|\mathbf{q}|^{b}}{1+|\mathbf{q}|^{b}} \hat{\psi}^{0} d \mathbf{q} d \mathbf{x} \leq C(\alpha) .
\end{aligned}
$$


104 GLOBAL EXISTENCE OF THE REGULARIZED HOOKEAN DUMBBELL MODEL

Note also that $\frac{M^{\beta} U}{1+|\mathbf{q}|^{b}} \in Y_{b}^{\beta}$, under the assumptions $\left(\mathrm{A}_{1}\right)$ and $\left(\mathrm{A}_{3}\right)$. Similarly we choose $\varphi=\frac{M^{\beta} U}{1+|\mathbf{q}|^{b}}$ in (4.16). Then, on recalling (3.2) and (4.13), we find

$$
\begin{aligned}
& \int_{\Omega \times D} G_{c}\left(\hat{\psi}^{n}-\hat{\psi}^{n-1}\right) U d \mathbf{q} d \mathbf{x}+\triangle t \frac{\beta}{2} \int_{\Omega \times D} A\left(\hat{\mathbf{u}}^{n}\right)|\mathbf{q}|^{2} \hat{\psi}^{n} U d \mathbf{q} d \mathbf{x} \\
& +\triangle t \frac{b}{2} \int_{\Omega \times D} \frac{|\mathbf{q}|^{b}}{1+|\mathbf{q}|^{b}}\left(A\left(\hat{\mathbf{u}}^{n}\right) \hat{\psi}^{n}-A\left(\hat{\mathbf{u}}^{n-1}\right) \hat{\psi}^{n-1}\right) U d \mathbf{q} d \mathbf{x} \\
& +\frac{1-\beta}{2 \lambda} \Delta t \int_{\Omega \times D}|\mathbf{q}|^{2} \hat{\psi}^{n} d \mathbf{q} d \mathbf{x}-\triangle t \int_{\Omega} \mathbf{C}\left(\hat{\psi}^{n}\right): \nabla_{x}\left(\mathbf{J}_{\alpha}^{x} \hat{\mathbf{u}}^{n}\right) d \mathbf{x} \\
& =\frac{(\triangle t)^{2}}{\lambda} \int_{\Omega \times D}|\mathbf{q}|^{2} \hat{\psi}^{n} d \mathbf{q} d \mathbf{x}-\frac{\beta}{2 \lambda} \triangle t \int_{\Omega \times D} W_{c}|\mathbf{q}|^{2} \hat{\psi}^{n} d \mathbf{q} d \mathbf{x} \\
& +\frac{d}{2 \lambda} \triangle t \int_{\Omega \times D} W_{c} \hat{\psi}^{n} d \mathbf{q} d \mathbf{x} .
\end{aligned}
$$

Here, we have used Lemma 3.3, which is a crucial point in the above derivation. In (4.15), we choose $\mathbf{w}=\hat{\mathbf{u}}^{n} \in \mathbf{V}$ and obtain, using (4.17) and (4.20),

$$
\begin{aligned}
& \int_{\Omega}\left|\hat{\mathbf{u}}^{n}\right|^{2} d \mathbf{x}+\int_{\Omega}\left|\hat{\mathbf{u}}^{n}-\hat{\mathbf{u}}^{n-1}\right|^{2} d \mathbf{x}+2 \nu \Delta t \int_{\Omega}\left|\nabla_{x} \hat{\mathbf{u}}^{n}\right|^{2} d \mathbf{x} \\
= & \int_{\Omega}\left|\hat{\mathbf{u}}^{n-1}\right|^{2} d \mathbf{x}-2 \kappa \omega \Delta t \int_{\Omega} \mathbf{C}\left(\hat{\psi}^{n}\right): \nabla_{x}\left(\mathbf{J}_{\alpha}^{x} \hat{\mathbf{u}}^{n}\right) d \mathbf{x},
\end{aligned}
$$

where we have used the simple identity

$$
2 a(a-b)=a^{2}+(a-b)^{2}-b^{2} \forall a, b \in \mathbb{R} .
$$

Multiplying (4.28) by $2 \kappa \omega$, then combining the resulting equation with (4.29), and using (4.13), (4.27), we derive

$$
\begin{aligned}
& \int_{\Omega}\left|\hat{\mathbf{u}}^{n}\right|^{2} d \mathbf{x}+\int_{\Omega}\left|\hat{\mathbf{u}}^{n}-\hat{\mathbf{u}}^{n-1}\right|^{2} d \mathbf{x}+2 \nu \Delta t \int_{\Omega}\left|\nabla_{x} \hat{\mathbf{u}}^{n}\right|^{2} d \mathbf{x} \\
& +2 \kappa \omega \int_{\Omega \times D} G_{c} \hat{\psi}^{n} U d \mathbf{q} d \mathbf{x}+\beta \kappa \omega \triangle t \int_{\Omega \times D} A\left(\hat{\mathbf{u}}^{n}\right)|\mathbf{q}|^{2} \hat{\psi}^{n} U d \mathbf{q} d \mathbf{x} \\
& +\frac{1-\beta}{\lambda} \kappa \omega \Delta t \int_{\Omega \times D}|\mathbf{q}|^{2} \hat{\psi}^{n} d \mathbf{q} d \mathbf{x}+\frac{\beta}{\lambda} \kappa \omega \Delta t \int_{\Omega \times D} W_{c}|\mathbf{q}|^{2} \hat{\psi}^{n} d \mathbf{q} d \mathbf{x} \\
& +b \kappa \omega \triangle t \int_{\Omega \times D} \frac{|\mathbf{q}|^{b}}{1+|\mathbf{q}|^{b}} A\left(\hat{\mathbf{u}}^{n}\right) \hat{\psi}^{n} U d \mathbf{q} d \mathbf{x} \\
& \leq \int_{\Omega}\left|\hat{\mathbf{u}}^{n-1}\right|^{2} d \mathbf{x}+2 \kappa \omega \int_{\Omega \times D} G_{c} \hat{\psi}^{n-1} U d \mathbf{q} d \mathbf{x} \\
& +b \kappa \omega \triangle t \int_{\Omega \times D} \frac{|\mathbf{q}|^{b}}{1+|\mathbf{q}|^{b}} A\left(\hat{\mathbf{u}}^{n-1}\right) \hat{\psi}^{n-1} U d \mathbf{q} d \mathbf{x}+C(\alpha) \triangle t .
\end{aligned}
$$


Summing the above estimate over all $n$, and using (3.21), (4.10) and (4.14) yields

$$
\begin{aligned}
\max _{n=0 \rightarrow N} \int_{\Omega}\left|\hat{\mathbf{u}}^{n}\right|^{2} d \mathbf{x}+\sum_{n=1}^{N} \int_{\Omega}\left|\hat{\mathbf{u}}^{n}-\hat{\mathbf{u}}^{n-1}\right|^{2} d \mathbf{x}+2 \nu \sum_{n=0}^{N} \Delta t \int_{\Omega}\left|\nabla_{x} \hat{\mathbf{u}}^{n}\right|^{2} d \mathbf{x} \\
+2 \kappa \omega \max _{n=0 \rightarrow N} \int_{\Omega \times D} G_{c} \hat{\psi}^{n} U d \mathbf{q} d \mathbf{x}+\beta \kappa \omega \sum_{n=1}^{N} \triangle t \int_{\Omega \times D} A\left(\hat{\mathbf{u}}^{n}\right)|\mathbf{q}|^{2} \hat{\psi}^{n} U d \mathbf{q} d \mathbf{x} \\
\quad+\frac{1-\beta}{\lambda} \kappa \omega \sum_{n=1}^{N} \Delta t \int_{\Omega \times D}|\mathbf{q}|^{2} \hat{\psi}^{n} d \mathbf{q} d \mathbf{x}+\frac{\beta}{\lambda} \kappa \omega \sum_{n=1}^{N} \triangle t \int_{\Omega \times D} W_{c}|\mathbf{q}|^{2} \hat{\psi}^{n} d \mathbf{q} d \mathbf{x} \\
\quad+b \kappa \omega \max _{n=0 \rightarrow N} \triangle t \int_{\Omega \times D} \frac{|\mathbf{q}|^{b}}{1+|\mathbf{q}|^{b}} A\left(\hat{\mathbf{u}}^{n}\right) \hat{\psi}^{n} U d \mathbf{q} d \mathbf{x} \leq C(\alpha) .
\end{aligned}
$$

If we choose $\varphi=\hat{\psi}^{n} \in Y_{b}^{\beta}$ in (4.16), then from (4.24) and (4.30) we get

$$
\begin{aligned}
& \left(1-\frac{2 b(b-1+d)+d}{2 \lambda} \triangle t\right) \int_{\Omega \times D}\left(1+|\mathbf{q}|^{b}\right) G_{c} \frac{\left|\hat{\psi}^{n}\right|^{2}}{M^{\beta}} d \mathbf{q} d \mathbf{x} \\
& +2 \triangle t \int_{\Omega \times D}\left(1+|\mathbf{q}|^{b}\right)\left(\frac{W_{c}}{2 \lambda} M^{\beta}\left|\nabla_{q} \frac{\hat{\psi}^{n}}{M^{\beta}}\right|^{2}+\varepsilon M^{\beta}\left|\nabla_{x} \frac{\hat{\psi}^{n}}{M^{\beta}}\right|^{2}\right) d \mathbf{q} d \mathbf{x} \\
& +\frac{\beta(1-\beta)}{2 \lambda} \triangle t \int_{\Omega \times D}\left(1+|\mathbf{q}|^{b}\right)|\mathbf{q}|^{2} \frac{\left|\hat{\psi}^{n}\right|^{2}}{M^{\beta}} d \mathbf{q} d \mathbf{x}+\frac{b \beta \triangle t}{2 \lambda} \int_{\Omega \times D} W_{c}|\mathbf{q}|^{b} \frac{\left.\hat{\psi}^{n}\right|^{2}}{M^{\beta}} d \mathbf{q} d \mathbf{x} \\
& +\frac{b(1-\beta)}{2 \lambda} \triangle t \int_{\Omega \times D}|\mathbf{q}|^{b} \frac{\left|\hat{\psi}^{n}\right|^{2}}{M^{\beta}} d \mathbf{q} d \mathbf{x}+\int_{\Omega \times D}\left(1+|\mathbf{q}|^{b}\right) G_{c} \frac{\left|\hat{\psi}^{n}-\hat{\psi}^{n-1}\right|^{2}}{M^{\beta}} d \mathbf{q} d \mathbf{x} \\
& \leq \int_{\Omega \times D}\left(1+|\mathbf{q}|^{b}\right) G_{c} \frac{\left|\hat{\psi}^{n-1}\right|^{2}}{M^{\beta}} d \mathbf{q} d \mathbf{x}+\Delta t \frac{b}{2} \int_{\Omega \times D}|\mathbf{q}|^{b} A\left(\hat{\mathbf{u}}^{n-1}\right) \frac{\hat{\psi}^{n-1} \hat{\psi}^{n}}{M^{\beta}} d \mathbf{q} d \mathbf{x} .
\end{aligned}
$$

Moreover, by using the Hölder inequality, we have from (3.21), (4.31) and (4.13) that

$$
\begin{aligned}
& \Delta t \frac{b}{2} \int_{\Omega \times D}|\mathbf{q}|^{b} A\left(\hat{\mathbf{u}}^{n-1}\right) \frac{\hat{\psi}^{n-1} \hat{\psi}^{n}}{M^{\beta}} d \mathbf{q} d \mathbf{x} \\
\leq & b \lambda\left(A\left(\hat{\mathbf{u}}^{n-1}\right)\right)^{2} \triangle t \int_{\Omega \times D}|\mathbf{q}|^{b} \frac{\left|\hat{\psi}^{n-1}\right|^{2}}{M^{\beta}} d \mathbf{q} d \mathbf{x}+\frac{b}{16 \lambda} \Delta t \int_{\Omega \times D}|\mathbf{q}|^{b} \frac{\left|\hat{\psi}^{n}\right|^{2}}{M^{\beta}} d \mathbf{q} d \mathbf{x} \\
\leq & C(\alpha) \triangle t \int_{\Omega \times D}\left(1+|\mathbf{q}|^{b}\right) G_{c} \frac{\left|\hat{\psi}^{n-1}\right|^{2}}{M^{\beta}} d \mathbf{q} d \mathbf{x}+\frac{b}{16 \lambda} \Delta t \int_{\Omega \times D}|\mathbf{q}|^{b} \frac{\left|\hat{\psi}^{n}\right|^{2}}{M^{\beta}} d \mathbf{q} d \mathbf{x} .
\end{aligned}
$$

Let $\triangle t<\min \left(1, \frac{\lambda}{2 b(b-1+d)+d}\right)$. Then

$$
1<\frac{1}{1-\frac{2 b(b-1+d)+d}{2 \lambda} \triangle t} \leq 1+\frac{2 b(b-1+d)+d}{\lambda} \triangle t
$$


106 GLOBAL EXISTENCE OF THE REGULARIZED HOOKEAN DUMBBELL MODEL

Thus the above three relations yield

$$
\begin{aligned}
& \int_{\Omega \times D}\left(1+|\mathbf{q}|^{b}\right) G_{c} \frac{\left|\hat{\psi}^{n}\right|^{2}}{M^{\beta}} d \mathbf{q} d \mathbf{x}+\int_{\Omega \times D}\left(1+|\mathbf{q}|^{b}\right) G_{c} \frac{\left|\hat{\psi}^{n}-\hat{\psi}^{n-1}\right|^{2}}{M^{\beta}} d \mathbf{q} d \mathbf{x} \\
& +2 \Delta t \int_{\Omega \times D}\left(1+|\mathbf{q}|^{b}\right)\left(\frac{W_{c}}{2 \lambda} M^{\beta}\left|\nabla_{q} \frac{\hat{\psi}^{n}}{M^{\beta}}\right|^{2}+\varepsilon M^{\beta}\left|\nabla_{x} \frac{\hat{\psi}^{n}}{M^{\beta}}\right|^{2}\right) d \mathbf{q} d \mathbf{x} \\
& +\frac{b(1-2 \beta)}{4 \lambda} \Delta t \int_{\Omega \times D}|\mathbf{q}|^{b} \frac{\left|\hat{\psi}^{n}\right|^{2}}{M^{\beta}} d \mathbf{q} d \mathbf{x}+\frac{b \beta \triangle t}{2 \lambda} \int_{\Omega \times D} W_{c}|\mathbf{q}|^{b} \frac{\left|\hat{\psi}^{n}\right|^{2}}{M^{\beta}} d \mathbf{q} d \mathbf{x} \\
& +\frac{\beta(1-\beta)}{2 \lambda} \Delta t \int_{\Omega \times D}\left(1+|\mathbf{q}|^{b}\right)|\mathbf{q}|^{2} \frac{\left|\hat{\psi}^{n}\right|^{2}}{M^{\beta}} d \mathbf{q} d \mathbf{x} \\
& \leq(1+C(\alpha) \triangle t) \int_{\Omega \times D}\left(1+|\mathbf{q}|^{b}\right) G_{c} \frac{\left|\hat{\psi}^{n-1}\right|^{2}}{M^{\beta}} d \mathbf{q} d \mathbf{x} .
\end{aligned}
$$

Adding up the above estimate for all $n$, using Lemma 4.2 and noticing also (4.10) and (4.14), we find

$$
\begin{aligned}
\max _{n=0 \rightarrow N}\left[\int_{\Omega \times D}\left(1+|\mathbf{q}|^{b}\right) G_{c} \frac{\left|\hat{\psi}^{n}\right|^{2}}{M^{\beta}} d \mathbf{q} d \mathbf{x}\right]+\sum_{n=1}^{N} \int_{\Omega \times D}\left(1+|\mathbf{q}|^{b}\right) G_{c} \frac{\left|\hat{\psi}^{n}-\hat{\psi}^{n-1}\right|^{2}}{M^{\beta}} d \mathbf{q} d \mathbf{x} \\
+2 \sum_{n=1}^{N} \triangle t \int_{\Omega \times D}\left(1+|\mathbf{q}|^{b}\right)\left(\frac{W_{c}}{2 \lambda} M^{\beta}\left|\nabla_{q} \frac{\hat{\psi}^{n}}{M^{\beta}}\right|^{2}+\varepsilon M^{\beta}\left|\nabla_{x} \frac{\hat{\psi}^{n}}{M^{\beta}}\right|^{2}\right) d \mathbf{q} d \mathbf{x} \\
+\frac{b(1-2 \beta)}{4 \lambda} \sum_{n=1}^{N} \triangle t \int_{\Omega \times D}|\mathbf{q}|^{b} \frac{\left|\hat{\psi}^{n}\right|^{2}}{M^{\beta}} d \mathbf{q} d \mathbf{x}+\frac{b \beta}{2 \lambda} \sum_{n=1}^{N} \triangle t \int_{\Omega \times D} W_{c}|\mathbf{q}|^{b} \frac{\left|\hat{\psi}^{n}\right|^{2}}{M^{\beta}} d \mathbf{q} d \mathbf{x} \\
+\frac{\beta(1-\beta)}{2 \lambda} \sum_{n=0}^{N} \triangle t \int_{\Omega \times D}\left(1+|\mathbf{q}|^{b}\right)|\mathbf{q}|^{2} \frac{\left|\hat{\psi}^{n}\right|^{2}}{M^{\beta}} d \mathbf{q} d \mathbf{x} \leq C(\alpha, T) .
\end{aligned}
$$

Next we turn to estimating $\mathbf{C}\left(\hat{\psi}^{n}\right)$ in view of the Carlson inequality (3.28). Observing (2.5) and (3.28) together with the Hölder inequality implies that

$$
\begin{aligned}
& \int_{\Omega}\left|\mathbf{C}\left(\hat{\psi}^{n}\right)\right|^{2} d \mathbf{x} \leq\left.\left.\int_{\Omega}\left|\int_{D}\right| \mathbf{q}\right|^{2} \hat{\psi}^{n} d \mathbf{q}\right|^{2} d \mathbf{x} \\
& \leq \pi^{d} \int_{\Omega}\left(\prod_{A_{d}} \int_{D} \prod_{i} q_{i}^{2 b_{i}}|\mathbf{q}|^{4}\left|\hat{\psi}^{n}\right|^{2} d \mathbf{q}\right)^{\frac{1}{2^{d}}} d \mathbf{x} \\
& \leq \pi^{d} \int_{\Omega} \prod_{A_{d}}\left(\int_{D}|\mathbf{q}|^{4+2 \sum_{i=1}^{d} b_{i}}\left|\hat{\psi}^{n}\right|^{2} d \mathbf{q}\right)^{\frac{1}{2^{d}}} d \mathbf{x} \\
& \leq \pi^{d} \prod_{A_{d}}\left(\int_{\Omega \times D}|\mathbf{q}|^{4+2 \sum_{i=1}^{d} b_{i}}\left|\hat{\psi}^{n}\right|^{2} d \mathbf{q} d \mathbf{x}\right)^{\frac{1}{2^{d}}} .
\end{aligned}
$$

This shows that when $d=2$,

$$
\begin{aligned}
\int_{\Omega}\left|\mathbf{C}\left(\hat{\psi}^{n}\right)\right|^{2} d \mathbf{x} \leq & \pi^{2}\left(\int_{\Omega \times D}|\mathbf{q}|^{4}\left|\hat{\psi}^{n}\right|^{2} d \mathbf{q} d \mathbf{x}\right)^{\frac{1}{4}}\left(\int_{\Omega \times D}|\mathbf{q}|^{6}\left|\hat{\psi}^{n}\right|^{2} d \mathbf{q} d \mathbf{x}\right)^{\frac{1}{2}} \\
& \cdot\left(\int_{\Omega \times D}|\mathbf{q}|^{8}\left|\hat{\psi}^{n}\right|^{2} d \mathbf{q} d \mathbf{x}\right)^{\frac{1}{4}},
\end{aligned}
$$


and when $d=3$,

$$
\begin{aligned}
\int_{\Omega}\left|\mathbf{C}\left(\hat{\psi}^{n}\right)\right|^{2} d \mathbf{x} \leq & \pi^{3}\left(\int_{\Omega \times D}|\mathbf{q}|^{4}\left|\hat{\psi}^{n}\right|^{2} d \mathbf{q} d \mathbf{x}\right)^{\frac{1}{8}}\left(\int_{\Omega \times D}|\mathbf{q}|^{6}\left|\hat{\psi}^{n}\right|^{2} d \mathbf{q} d \mathbf{x}\right)^{\frac{3}{8}} \\
& \cdot\left(\int_{\Omega \times D}|\mathbf{q}|^{8}\left|\hat{\psi}^{n}\right|^{2} d \mathbf{q} d \mathbf{x}\right)^{\frac{3}{8}}\left(\int_{\Omega \times D}|\mathbf{q}|^{10}\left|\hat{\psi}^{n}\right|^{2} d \mathbf{q} d \mathbf{x}\right)^{\frac{1}{8}} .
\end{aligned}
$$

Therefore, using (4.34)-(4.35) with Assumption $\left(\mathrm{A}_{1}\right)$ and (4.33), we have

$$
\max _{n=0 \rightarrow N}\left[\int_{\Omega}\left|\mathbf{C}\left(\hat{\psi}^{n}\right)\right|^{2} d \mathbf{x}\right] \leq C(\alpha, T) .
$$

In the following we come to estimate the time difference of $\hat{\mathbf{u}}^{n}$. Our main method is based on the Gagliardo-Nirenberg inequality and the Helmholtz-Stokes mollifiers. For given $\epsilon>0$, by using Young's inequality with $\epsilon$ and (3.20) and (4.20), we have for (4.15) $\forall \mathbf{w} \in \mathbf{V}$,

$$
\begin{aligned}
\int_{\Omega} \frac{\hat{\mathbf{u}}^{n}-\hat{\mathbf{u}}^{n-1}}{\Delta t} \cdot \mathbf{w} d \mathbf{x}= & \int_{\Omega}\left[\left(\hat{\mathbf{u}}^{n-1} \cdot \nabla_{x}\right) \mathbf{w}\right] \cdot \hat{\mathbf{u}}^{n} d \mathbf{x}-\nu \int_{\Omega} \nabla_{x} \hat{\mathbf{u}}^{n}: \nabla_{x} \mathbf{w} d \mathbf{x} \\
& -\kappa \omega \int_{\Omega} \mathbf{C}\left(\hat{\psi}^{n}\right): \nabla_{x}\left(\mathbf{w}_{\alpha}\right) d \mathbf{x} \\
\leq & C \int_{\Omega}\left(\left|\hat{\mathbf{u}}^{n-1}\right|^{2}\left|\hat{\mathbf{u}}^{n}\right|^{2}+\left|\nabla_{x} \hat{\mathbf{u}}^{n}\right|^{2}+\left|\mathbf{C}\left(\hat{\psi}^{n}\right)\right|^{2}\right) d \mathbf{x}+\epsilon \int_{\Omega}\left|\nabla_{x} \mathbf{w}\right|^{2} d \mathbf{x} .
\end{aligned}
$$

Application of the Gagliardo-Nirenberg inequaliy (3.30) yields

$$
\begin{aligned}
\int_{\Omega}\left|\hat{\mathbf{u}}^{n-1}\right|^{2}\left|\hat{\mathbf{u}}^{n}\right|^{2} d \mathbf{x} & \leq\left(\int_{\Omega}\left|\hat{\mathbf{u}}^{n-1}\right|^{4} d \mathbf{x}\right)^{\frac{1}{2}}\left(\int_{\Omega}\left|\hat{\mathbf{u}}^{n}\right|^{4} d \mathbf{x}\right)^{\frac{1}{2}} \leq \sum_{m=n-1}^{n} \int_{\Omega}\left|\hat{\mathbf{u}}^{m}\right|^{4} d \mathbf{x} \\
& \leq C \sum_{m=n-1}^{n}\left[\left(\int_{\Omega}\left|\hat{\mathbf{u}}^{m}\right|^{2} d \mathbf{x}\right)^{2-\frac{d}{2}}\left(\int_{\Omega}\left|\nabla_{x} \hat{\mathbf{u}}^{m}\right|^{2} d \mathbf{x}\right)^{\frac{d}{2}}\right] .
\end{aligned}
$$

This and (4.37) imply

$$
\begin{aligned}
& {\left[\int_{\Omega} \frac{\hat{\mathbf{u}}^{n}-\hat{\mathbf{u}}^{n-1}}{\Delta t} \cdot \mathbf{w} d \mathbf{x}-\epsilon \int_{\Omega}\left|\nabla_{x} \mathbf{w}\right|^{2} d \mathbf{x}\right]^{\frac{2}{d}} } \\
\leq & C \sum_{m=n-1}^{n}\left(\int_{\Omega}\left|\hat{\mathbf{u}}^{m}\right|^{2} d \mathbf{x}\right)^{\frac{4}{d}-1} \int_{\Omega}\left|\nabla_{x} \hat{\mathbf{u}}^{m}\right|^{2} d \mathbf{x} \\
& +C\left[\int_{\Omega}\left|\nabla_{x} \hat{\mathbf{u}}^{n}\right|^{2} d \mathbf{x}\right]^{\frac{2}{d}}+\left[\int_{\Omega}\left|\mathbf{C}\left(\hat{\psi}^{n}\right)\right|^{2} d \mathbf{x}\right]^{\frac{2}{d}} .
\end{aligned}
$$

By using (4.36) and (4.31), we furthermore have

$$
\begin{aligned}
& {\left[\int_{\Omega} \frac{\hat{\mathbf{u}}^{n}-\hat{\mathbf{u}}^{n-1}}{\triangle t} \cdot \mathbf{w} d \mathbf{x}-\epsilon \int_{\Omega}\left|\nabla_{x} \mathbf{w}\right|^{2} d \mathbf{x}\right]^{\frac{2}{d}} } \\
\leq & C(\alpha) \sum_{m=n-1}^{n} \int_{\Omega}\left|\nabla_{x} \hat{\mathbf{u}}^{m}\right|^{2} d \mathbf{x}+C\left[\int_{\Omega}\left|\nabla_{x} \hat{\mathbf{u}}^{n}\right|^{2} d \mathbf{x}\right]^{\frac{2}{d}}+C(\alpha, T) .
\end{aligned}
$$


Summing the above estimate over all $n$ and noticing (4.31) yield

$$
\begin{aligned}
& \sum_{n=1}^{N} \Delta t\left[\int_{\Omega} \frac{\hat{\mathbf{u}}^{n}-\hat{\mathbf{u}}^{n-1}}{\Delta t} \cdot \mathbf{w} d \mathbf{x}-\epsilon \int_{\Omega}\left|\nabla_{x} \mathbf{w}\right|^{2} d \mathbf{x}\right]^{\frac{2}{d}} \\
\leq & C(\alpha) \sum_{n=1}^{N} \sum_{m=n-1}^{n} \Delta t \int_{\Omega}\left|\nabla_{x} \hat{\mathbf{u}}^{m}\right|^{2} d \mathbf{x}+C\left[\sum_{n=1}^{N} \triangle t \int_{\Omega}\left|\nabla_{x} \hat{\mathbf{u}}^{n}\right|^{2} d \mathbf{x}\right]^{\frac{2}{d}}+C(\alpha, T) \\
\leq & C(\alpha, T) .
\end{aligned}
$$

If we choose $\mathbf{w}=\mathbf{S}_{\gamma}\left(\frac{\hat{\mathbf{u}}^{n}-\hat{\mathbf{u}}^{n-1}}{\triangle t}\right) \in \mathbf{V}$ in (4.15), then by the above relation, we have

$$
\begin{aligned}
& \sum_{n=1}^{N} \triangle t\left[\int_{\Omega} \frac{\hat{\mathbf{u}}^{n}-\hat{\mathbf{u}}^{n-1}}{\triangle t} \cdot \mathbf{S}_{\gamma}\left(\frac{\hat{\mathbf{u}}^{n}-\hat{\mathbf{u}}^{n-1}}{\triangle t}\right) d \mathbf{x}\right. \\
& \left.-\epsilon \int_{\Omega}\left|\nabla_{x} \mathbf{S}_{\gamma}\left(\frac{\hat{\mathbf{u}}^{n}-\hat{\mathbf{u}}^{n-1}}{\triangle t}\right)\right|^{2} d \mathbf{x}\right]^{\frac{2}{d}} \\
& \leq C(\alpha, T) .
\end{aligned}
$$

With the choice of $\epsilon<\frac{\gamma}{2}$, by the above relation and (3.23) we finally conclude that

$$
\sum_{n=1}^{N} \triangle t\left\|\mathbf{S}_{\gamma}\left(\frac{\hat{\mathbf{u}}^{n}-\hat{\mathbf{u}}^{n-1}}{\triangle t}\right)\right\|_{H^{1}(\Omega)}^{\frac{4}{d}} \leq C(\alpha, T) .
$$

REMARK 4.3. As in the first item of Remark 4.2, there are some techniques in constructing the time-discretized problem (4.10)-(4.12) which are different from that in [2]. Thus the difficulties are different in deriving a priori estimates for $\mathbf{u}^{n}, \psi^{n}$. Especially, in our estimate derivations, we can easily tackle the term on the left-hand side of (4.32) and obtain estimates of $\hat{\psi}^{n}$ (see (4.33)), while in the corresponding derivations in [2], the authors had to use the boundedness condition from $D$ and then failed to deal with the Hookean dumbbell model.

4.3. Rothe functions and a priori estimates. We can now define Rothe functions obtained from $\hat{\mathbf{u}}^{n}$ by piecewise constant and piecewise linear interpolation with respect to time $t$, respectively. Let

$$
\hat{\mathbf{u}}^{\triangle t,+}(\cdot, t):=\hat{\mathbf{u}}^{n}(\cdot), \quad \hat{\mathbf{u}}^{\triangle t,-}(\cdot, t):=\hat{\mathbf{u}}^{n-1}(\cdot), \quad t \in\left(t_{n-1}, t_{n}\right], n \geq 1,
$$

and

$$
\hat{\mathbf{u}}^{\triangle t}(\cdot, t):=\frac{t-t_{n-1}}{\triangle t} \hat{\mathbf{u}}^{n}(\cdot)+\frac{t_{n}-t}{\triangle t} \hat{\mathbf{u}}^{n-1}(\cdot), \quad t \in\left[t_{n-1}, t_{n}\right], \quad n \geq 1
$$

Then

$$
\hat{\mathbf{u}}^{\triangle t}-\hat{\mathbf{u}}^{\triangle t, \pm}=\left(t-t_{n}^{ \pm}\right) \frac{\partial \hat{\mathbf{u}}^{\triangle t}}{\partial t}, \quad t \in\left(t_{n-1}, t_{n}\right], n \geq 1,
$$

where $t_{n}^{+}:=t_{n}$ and $t_{n}^{-}:=t_{n-1}$. For brevity, we will sometimes use the notation $\hat{\mathbf{u}}^{\triangle t(, \pm)}(\cdot, t)$ to mean $\hat{\mathbf{u}}^{\triangle t}, \hat{\mathbf{u}}^{\triangle t,+}$ or $\hat{\mathbf{u}}^{\triangle t,-}$. We also define $\hat{\psi}^{\triangle t(, \pm)}(\cdot, \cdot, t)$ for $\left\{\hat{\psi}^{n}\right\}_{n=0}^{N}$ in an analogous way. These Rothe functions are intended to be approximations of the solution to the modified model in some suitable function spaces. 
Now piecewise constant interpolation of (4.11)-(4.12) over $[0, T]$ yields

$$
\begin{aligned}
& \int_{0}^{T}<\frac{\partial \hat{\mathbf{u}}^{\Delta t}}{\partial t}, \mathbf{w}>d t+\int_{0}^{T} \int_{\Omega}\left[\left[\left(\hat{\mathbf{u}}^{\Delta t,-} \cdot \nabla_{x}\right) \hat{\mathbf{u}}^{\Delta t,+}\right] \cdot \mathbf{w}+\nu \nabla_{x} \hat{\mathbf{u}}^{\Delta t,+}: \nabla_{x} \mathbf{w}\right] d \mathbf{x} d t \\
= & -\kappa \omega \int_{0}^{T} \int_{\Omega} \mathbf{C}\left(\hat{\psi}^{\Delta t,+}\right): \nabla_{x} \mathbf{w}_{\alpha} d \mathbf{x} d t, \quad \forall \mathbf{w} \in L^{\frac{4}{4-d}}(0, T ; \mathbf{V}), \\
& \int_{0}^{T} \int_{\Omega \times D}\left(1+|\mathbf{q}|^{b}\right) G_{c} \frac{\hat{\psi}^{\Delta t,+}-\hat{\psi}^{\Delta t,-}}{\Delta t} \frac{\varphi}{M^{\beta}} d \mathbf{q} d \mathbf{x} d t \\
& +\frac{b}{2} \int_{0}^{T} \int_{\Omega \times D}|\mathbf{q}|^{b}\left[A\left(\hat{\mathbf{u}}^{\Delta t,+}\right) \hat{\psi}^{\Delta t,+}-A\left(\hat{\mathbf{u}}^{\Delta t,-}\right) \hat{\psi}^{\Delta t,-}\right] \frac{\varphi}{M^{\beta}} d \mathbf{q} d \mathbf{x} d t \\
& +\int_{0}^{T} \int_{\Omega \times D}\left[\frac{W_{c}}{2 \lambda} M^{\beta} \nabla_{q} \frac{\hat{\psi}^{\Delta t,+}}{M^{\beta}}+\frac{1-\beta}{2 \lambda} \mathbf{q} \hat{\psi}^{\Delta t,+}-\left[\nabla_{x}\left(\mathbf{J}_{\alpha}^{x} \hat{\mathbf{u}}^{\Delta t,+}\right) \mathbf{q}\right] \hat{\psi}^{\Delta t,+}\right] \\
& \cdot \nabla_{q}\left(\left(1+|\mathbf{q}|^{b}\right) \frac{\varphi}{M^{\beta}}\right) d \mathbf{q} d \mathbf{x} d t+\frac{\beta}{2} \int_{0}^{T} \int_{\Omega \times D}\left\|\nabla_{x}\left(\mathbf{J}_{\alpha}^{x} \hat{\mathbf{u}}^{\Delta t,+}\right)\right\|_{\infty}|\mathbf{q}|^{2}\left(1+|\mathbf{q}|^{b}\right) \\
& . \frac{\hat{\psi}^{\Delta t,+} \varphi}{M^{\beta}} d \mathbf{q} d \mathbf{x} d t+\int_{0}^{T} \int_{\Omega \times D}\left(1+|\mathbf{q}|^{b}\right) \\
& \cdot\left(\varepsilon \nabla_{x} \hat{\psi}^{\Delta t,+}-\hat{\mathbf{u}}^{\Delta t,+} \hat{\psi}^{\Delta t,+}\right) \cdot \nabla_{x} \frac{\varphi}{M^{\beta}} d \mathbf{q} d \mathbf{x} d t=0, \forall \varphi \in L^{2}\left(0, T ; Y_{b}^{\beta}\right) .
\end{aligned}
$$

Moreover, (4.31), (4.33), (4.36) and (4.38) imply the following obvious estimates for the Rothe functions $\hat{\mathbf{u}}^{\Delta t(, \pm)}$ and $\hat{\psi}^{\Delta t(, \pm)}$.

$$
\begin{aligned}
& \sup _{t \in(0, T)}\left[\int_{\Omega}\left|\hat{\mathbf{u}}^{\Delta t(, \pm)}\right|^{2} d \mathbf{x}\right]+\int_{0}^{T} \int_{\Omega} \frac{\left|\hat{\mathbf{u}}^{\Delta t,+}-\hat{\mathbf{u}}^{\Delta t,-}\right|^{2}}{\Delta t} d \mathbf{x} d t \\
+ & 2 \nu \int_{0}^{T} \int_{\Omega}\left|\nabla_{x} \hat{\mathbf{u}}^{\Delta t(, \pm)}\right|^{2} d \mathbf{x} d t \leq C(\alpha), \\
& \sup _{t \in(0, T)}\left[\int_{\Omega \times D}\left(1+|\mathbf{q}|^{b}\right) G_{c} \frac{\left|\hat{\psi}^{\Delta t(, \pm)}\right|^{2}}{M^{\beta}} d \mathbf{q} d \mathbf{x}\right] \\
& +\int_{0}^{T} \int_{\Omega \times D}\left(1+|\mathbf{q}|^{b}\right) \frac{G_{c}}{M^{\beta}} \frac{\left|\hat{\psi}^{\Delta t,+}-\hat{\psi}^{\triangle t,-}\right|^{2}}{\triangle t} d \mathbf{q} d \mathbf{x} d t \\
& +\int_{0}^{T} \int_{\Omega \times D}\left(1+|\mathbf{q}|^{b}\right)\left(\frac{W_{c}}{\lambda} M^{\beta}\left|\nabla_{q} \frac{\hat{\psi}^{\Delta t,+}}{M^{\beta}}\right|^{2}+2 \varepsilon M^{\beta}\left|\nabla_{x} \frac{\hat{\psi}^{\Delta t,+}}{M^{\beta}}\right|^{2}\right) d \mathbf{q} d \mathbf{x} d t \\
& +\frac{\beta(1-\beta)}{2 \lambda} \int_{0}^{T} \int_{\Omega \times D}\left(1+|\mathbf{q}|^{b}\right)|\mathbf{q}|^{2} \frac{\left|\hat{\psi}^{\triangle t(, \pm)}\right|^{2}}{M^{\beta}} d \mathbf{q} d \mathbf{x} d t+\sup _{t \in(0, T)}\left[\int_{\Omega}\left|\mathbf{C}\left(\hat{\psi}^{\triangle t(, \pm)}\right)\right|^{2} d \mathbf{x}\right] \\
& +\int_{0}^{T}\left\|\mathbf{S}_{\gamma}\left(\frac{\partial \hat{\mathbf{u}}^{\Delta t}}{\partial t}\right)\right\|_{H^{1}(\Omega)}^{\frac{4}{d}} d t \leq C(\alpha, T) .
\end{aligned}
$$

4.4. The proof of Proposition 4.1. After obtaining a priori estimates for the sequences of the Rothe functions, we propose to establish the convergence of the Rothe functions and then conclude this subsection with the proof of Proposition 4.1. For convenience, here and below, we will identify $\left\{\hat{\mathbf{u}}^{\triangle t}, \hat{\psi}^{\triangle t}\right\}_{\triangle t}$ with its subsequence and we shall not state it any further. 
110 GLOBAL EXISTENCE OF THE REGULARIZED HOOKEAN DUMBBELL MODEL

Lemma 4.3. Suppose assumptions $\left(A_{1}\right)-\left(A_{3}\right)$. Then, for given $\alpha, \varepsilon \in$ $(0,1]$ there exist $\hat{\mathbf{u}} \in L^{\infty}\left(0, T ; \mathbf{L}^{2}(\Omega)\right) \cap L^{2}(0, T ; \mathbf{V}) \cap W^{1, \frac{4}{d}}\left(0, T ; \mathbf{V}^{\prime}\right), \hat{\psi} \in L^{2}\left(0, T ; Z_{b}^{\beta,+}\right)$ $\cap L^{\infty}\left(0, T ; L^{2}\left(\Omega \times D ;\left(1+|\mathbf{q}|^{b}\right) M^{-\beta}\right)\right)$, and a subsequence of the Rothe functions $\left\{\hat{\mathbf{u}}^{\triangle t}, \hat{\psi}^{\triangle t}\right\}_{\triangle t}$ satisfying

$$
\begin{gathered}
\left(1+|\mathbf{q}|^{b}\right)^{\frac{1}{2}} \frac{\hat{\psi}^{\Delta t(, \pm)}}{M^{\frac{\beta}{2}}} \stackrel{*}{\rightarrow}\left(1+|\mathbf{q}|^{b}\right)^{\frac{1}{2}} \frac{\hat{\psi}}{M^{\frac{\beta}{2}}} \text { in } L^{\infty}\left(0, T ; L^{2}(\Omega \times D)\right), \\
\beta\left(1+|\mathbf{q}|^{b}\right)^{\frac{1}{2}} \mathbf{q} \frac{\hat{\psi}^{\Delta t,+}}{M^{\frac{\beta}{2}}} \rightarrow \beta\left(1+|\mathbf{q}|^{b}\right)^{\frac{1}{2}} \mathbf{q} \frac{\hat{\psi}}{M^{\frac{\beta}{2}}} \text { in } L^{2}\left(0, T ; \mathbf{L}^{2}(\Omega \times D)\right), \\
\varepsilon\left(1+|\mathbf{q}|^{b}\right)^{\frac{1}{2}} M^{\frac{\beta}{2}} \nabla_{x}\left(\frac{\hat{\psi}^{\Delta t,+}}{M^{\beta}}\right) \rightarrow \varepsilon\left(1+|\mathbf{q}|^{b}\right)^{\frac{1}{2}} M^{\frac{\beta}{2}} \nabla_{x}\left(\frac{\hat{\psi}}{M^{\beta}}\right) \\
\text { in } L^{2}\left(0, T ; \mathbf{L}^{2}(\Omega \times D)\right), \\
\left(1+|\mathbf{q}|^{b}\right)^{\frac{1}{2}} W_{c}^{\frac{1}{2}} M^{\frac{\beta}{2}} \nabla_{q}\left(\frac{\hat{\psi}^{\triangle t,+}}{M^{\beta}}\right) \rightarrow\left(1+|\mathbf{q}|^{b}\right)^{\frac{1}{2}} W_{c}^{\frac{1}{2}} M^{\frac{\beta}{2}} \nabla_{q}\left(\frac{\hat{\psi}}{M^{\beta}}\right) \\
\text { in } L^{2}\left(0, T ; \mathbf{L}^{2}(\Omega \times D)\right), \\
\mathbf{C}\left(\hat{\psi}^{\Delta t(, \pm)}\right) \stackrel{*}{\rightarrow} \mathbf{C}(\hat{\psi}) \text { in } L^{\infty}\left(0, T ; \mathbf{L}^{2}(\Omega)\right),
\end{gathered}
$$

and

$$
\begin{gathered}
\hat{\mathbf{u}}^{\Delta t(, \pm)} \stackrel{*}{\rightarrow} \hat{\mathbf{u}} \text { in } L^{\infty}\left(0, T ; \mathbf{L}^{2}(\Omega)\right), \\
\hat{\mathbf{u}}^{\Delta t(, \pm)} \rightarrow \hat{\mathbf{u}} \text { in } L^{2}(0, T ; \mathbf{V}), \\
\mathbf{S}_{\gamma} \frac{\partial \hat{\mathbf{u}}^{\triangle t}}{\partial t} \rightarrow \mathbf{S}_{\gamma} \frac{\partial \hat{\mathbf{u}}}{\partial t} \text { in } L^{\frac{4}{d}}(0, T ; \mathbf{V}), \\
\hat{\mathbf{u}}^{\Delta t(, \pm)} \rightarrow \hat{\mathbf{u}} \text { in } L^{2}\left(0, T ; \mathbf{L}^{r}(\Omega)\right), \\
\mathbf{J}_{\alpha}^{x} \hat{\mathbf{u}}^{\triangle t(, \pm)} \rightarrow \mathbf{J}_{\alpha}^{x} \hat{\mathbf{u}} \text { in } L^{2}\left(0, T ; \mathbf{W}^{1, \infty}(\Omega)\right), \\
\mathbf{J}_{\alpha}^{x} \hat{\mathbf{u}}^{\triangle t(, \pm)} \stackrel{*}{\rightarrow} \mathbf{J}_{\alpha}^{x} \hat{\mathbf{u}} \text { in } L^{\infty}\left(0, T ; \mathbf{W}^{1, \infty}(\Omega)\right),
\end{gathered}
$$

as $\triangle t \rightarrow 0_{+}$, where $r \in[1, \infty)$ if $d=2$ and $r \in[1,6)$ if $d=3$.

The proof of this lemma is in Appendix A. Moreover, it follows immediately from (4.44)-(4.45) and Lemma 4.3 that

COROLlary 4.4. The limit function $(\hat{\mathbf{u}}, \hat{\psi})$ satisfies

$$
\begin{aligned}
& \sup _{t \in(0, T)}\left[\int_{\Omega \times D}\left(1+|\mathbf{q}|^{b}\right) \frac{|\hat{\psi}|^{2}}{M^{\beta}} d \mathbf{q} d \mathbf{x}\right]+\sup _{t \in(0, T)}\left[\int_{\Omega}|\mathbf{C}(\hat{\psi})|^{2} d \mathbf{x}\right] \\
& +\int_{0}^{T} \int_{\Omega \times D}\left(1+|\mathbf{q}|^{b}\right)\left(\frac{1}{\lambda} M^{\beta}\left|\nabla_{q} \frac{\hat{\psi}}{M^{\beta}}\right|^{2}+2 \varepsilon M^{\beta}\left|\nabla_{x} \frac{\hat{\psi}}{M^{\beta}}\right|^{2}\right) d \mathbf{q} d \mathbf{x} d t \\
& +\frac{\beta(1-\beta)}{2 \lambda} \int_{0}^{T} \int_{\Omega \times D}\left(1+|\mathbf{q}|^{b}\right)|\mathbf{q}|^{2} \frac{|\hat{\psi}|^{2}}{M^{\beta}} d \mathbf{q} d \mathbf{x} d t+\int_{0}^{T}\left\|\mathbf{S}_{\gamma}\left(\frac{\partial \hat{\mathbf{u}}}{\partial t}\right)\right\|_{H^{1}(\Omega)}^{\frac{4}{d}} d t \\
& \leq C(\alpha, T), \\
& \sup _{t \in(0, T)}\left[\int_{\Omega}|\hat{\mathbf{u}}|^{2} d \mathbf{x}\right]+2 \nu \int_{0}^{T} \int_{\Omega}\left|\nabla_{x} \hat{\mathbf{u}}\right|^{2} d \mathbf{x} d t \leq C(\alpha) .
\end{aligned}
$$


Proof of Proposition 4.1. In fact, it only remains to prove that the limit function $(\hat{\mathbf{u}}, \hat{\psi})$ in Lemma 4.3 satisfies (4.7)-(4.9). Now we first verify (4.7).

From (4.53), we have

$$
\begin{aligned}
\int_{0}^{T} & \int_{\Omega} \mathbf{w} \cdot \mathbf{S}_{\gamma} \frac{\partial \hat{\mathbf{u}}^{\Delta t}}{\partial t} d \mathbf{x} d t+\gamma \int_{0}^{T} \int_{\Omega} \nabla_{x} \mathbf{w} \cdot \nabla_{x} \mathbf{S}_{\gamma} \frac{\partial \hat{\mathbf{u}}^{\Delta t}}{\partial t} d \mathbf{x} d t \\
& \rightarrow \int_{0}^{T} \int_{\Omega} \mathbf{w} \cdot \mathbf{S}_{\gamma} \frac{\partial \hat{\mathbf{u}}}{\partial t} d \mathbf{x} d t+\gamma \int_{0}^{T} \int_{\Omega} \nabla_{x} \mathbf{w} \cdot \nabla_{x} \mathbf{S}_{\gamma} \frac{\partial \hat{\mathbf{u}}}{\partial t} d \mathbf{x} d t, \quad \forall \mathbf{w} \in L^{\frac{4}{4-d}}(0, T ; \mathbf{V}),
\end{aligned}
$$

as $\Delta t \rightarrow 0_{+}$. Then, by (3.22), this implies

$$
\lim _{\triangle t \rightarrow 0_{+}} \int_{0}^{T}<\frac{\partial \hat{\mathbf{u}}^{\Delta t}}{\partial t}, \mathbf{w}>d t=\int_{0}^{T}<\frac{\partial \hat{\mathbf{u}}}{\partial t}, \mathbf{w}>d t, \quad \forall \mathbf{w} \in L^{\frac{4}{4-d}}(0, T ; \mathbf{V}) .
$$

To get (4.7), another key point is to prove for any $\mathbf{w} \in L^{\frac{4}{4-d}}(0, T ; \mathbf{V})$,

$$
\lim _{\triangle t \rightarrow 0_{+}} \int_{0}^{T} \int_{\Omega}\left[\left(\hat{\mathbf{u}}^{\Delta t,-} \cdot \nabla_{x}\right) \hat{\mathbf{u}}^{\Delta t,+}\right] \cdot \mathbf{w} d \mathbf{x} d t=\int_{0}^{T} \int_{\Omega}\left[\left(\hat{\mathbf{u}} \cdot \nabla_{x}\right) \hat{\mathbf{u}}\right] \cdot \mathbf{w} d \mathbf{x} d t
$$

For any fixed $\tilde{\mathbf{w}} \in L^{\frac{4}{4-d}}(0, T ; \mathcal{V})$ and any fixed $\mathbf{w} \in L^{\frac{4}{4-d}}(0, T ; \mathbf{V})$, we have

$$
\begin{aligned}
& \int_{0}^{T} \int_{\Omega}\left[\left(\hat{\mathbf{u}}^{\Delta t,-} \cdot \nabla_{x}\right) \hat{\mathbf{u}}^{\Delta t,+}\right] \cdot \mathbf{w} d \mathbf{x} d t-\int_{0}^{T} \int_{\Omega}\left(\hat{\mathbf{u}} \cdot \nabla_{x}\right) \hat{\mathbf{u}} \cdot \mathbf{w} d \mathbf{x} d t \\
= & \int_{0}^{T} \int_{\Omega}\left[\left(\hat{\mathbf{u}}^{\Delta t,-} \cdot \nabla_{x}\right) \hat{\mathbf{u}}^{\Delta t,+}\right] \cdot(\mathbf{w}-\tilde{\mathbf{w}}) d \mathbf{x} d t+\int_{0}^{T} \int_{\Omega}\left[\left(\hat{\mathbf{u}} \cdot \nabla_{x}\right) \hat{\mathbf{u}}\right] \cdot(\tilde{\mathbf{w}}-\mathbf{w}) d \mathbf{x} d t \\
& +\int_{0}^{T} \int_{\Omega}\left[\left(\left(\hat{\mathbf{u}}^{\Delta t,-}-\hat{\mathbf{u}}\right) \cdot \nabla_{x}\right) \hat{\mathbf{u}}^{\Delta t,+}\right] \cdot \tilde{\mathbf{w}} d \mathbf{x} d t \\
& +\int_{0}^{T} \int_{\Omega}\left[\left(\hat{\mathbf{u}} \cdot \nabla_{x}\right)\left(\hat{\mathbf{u}}^{\Delta t,+}-\hat{\mathbf{u}}\right)\right] \cdot \tilde{\mathbf{w}} d \mathbf{x} d t .
\end{aligned}
$$

Noting that $\hat{\mathbf{u}}^{\Delta t(, \pm)} \in L^{\infty}\left(0, T ; \mathbf{L}^{2}(\Omega)\right) \cap L^{2}(0, T ; \mathbf{V})$, we then deduce that

$$
\left\|\hat{\mathbf{u}}^{\Delta t(, \pm)}\right\|_{L^{\frac{2}{\gamma}\left(0, T ; L^{r}(\Omega)\right)}} \leq C
$$

for $\gamma=d\left(\frac{1}{2}-\frac{1}{r}\right), r \in[2, \infty)$ if $d=2$ and $r \in[2,6]$ if $d=3$. Thus by (4.20) and (4.62), we derive

$$
\begin{aligned}
& \left|\int_{0}^{T} \int_{\Omega}\left[\left(\hat{\mathbf{u}}^{\Delta t,-} \cdot \nabla_{x}\right) \hat{\mathbf{u}}^{\Delta t,+}\right] \cdot(\mathbf{w}-\tilde{\mathbf{w}}) d \mathbf{x} d t\right| \\
= & \left|\int_{0}^{T} \int_{\Omega}\left[\left(\hat{\mathbf{u}}^{\Delta t,-} \cdot \nabla_{x}\right)(\mathbf{w}-\tilde{\mathbf{w}})\right] \cdot \hat{\mathbf{u}}^{\Delta t,+} d \mathbf{x} d t\right| \\
\leq & C\left\|\hat{\mathbf{u}}^{\Delta t,-}\right\|_{L^{\frac{8}{d}\left(0, T ; L^{4}(\Omega)\right)}}\left\|\hat{\mathbf{u}}^{\Delta t,+}\right\|_{L^{\frac{8}{d}}\left(0, T ; L^{4}(\Omega)\right)}\|\mathbf{w}-\tilde{\mathbf{w}}\|_{L^{\frac{4}{4-d}}(0, T ; V)} \\
\leq & C\|\mathbf{w}-\tilde{\mathbf{w}}\|_{L^{\frac{4}{4-d}}(0, T ; V)}
\end{aligned}
$$


112 GLOBAL EXISTENCE OF THE REGULARIZED HOOKEAN DUMBBELL MODEL

Analogously, we have

$$
\begin{aligned}
& \mid \int_{0}^{T} \int_{\Omega}\left[\left(\hat{\mathbf{u}} \cdot \nabla_{x} \hat{\mathbf{u}}\right] \cdot(\tilde{\mathbf{w}}-\mathbf{w}) d \mathbf{x} d t \mid \leq C\|\mathbf{w}-\tilde{\mathbf{w}}\|_{L^{\frac{4}{4-d}}(0, T ; V)},\right. \\
& \left|\int_{0}^{T} \int_{\Omega}\left[\left(\left(\hat{\mathbf{u}}^{\Delta t,-}-\hat{\mathbf{u}}\right) \cdot \nabla_{x}\right) \hat{\mathbf{u}}^{\Delta t,+}\right] \cdot \tilde{\mathbf{w}} d \mathbf{x} d t\right| \leq C\left\|\hat{\mathbf{u}}^{\Delta t,-}-\hat{\mathbf{u}}\right\|_{L^{2}\left(0, T ; L^{2}(\Omega)\right)}, \\
& \left|\int_{0}^{T} \int_{\Omega}\left[\left(\hat{\mathbf{u}} \cdot \nabla_{x}\right)\left(\hat{\mathbf{u}}^{\Delta t,+}-\hat{\mathbf{u}}\right)\right] \cdot \tilde{\mathbf{w}} d \mathbf{x} d t\right| \leq C\left\|\hat{\mathbf{u}}^{\Delta t,-}-\hat{\mathbf{u}}\right\|_{L^{2}\left(0, T ; L^{2}(\Omega)\right)} .
\end{aligned}
$$

By using these four relations, we can obtain (4.60) from (4.61) on noting (4.54) and the denseness of $\mathcal{V}$ in $\mathbf{V}$.

Therefore, by limit $\Delta t \rightarrow 0_{+}$in $(4.42)$, we have that $(\hat{\mathbf{u}}, \hat{\psi})$ satisfies $(4.7)$ in virtue of (3.20), (4.50), (4.52), (4.59)-(4.60). Moreover, (4.9) follows immediately from (4.10) and (4.13).

Next, we will verify (4.8) through (4.43). Since we do not have estimates for time derivative of $\psi_{\alpha, \beta, \varepsilon}^{\Delta t}$, we have to transfer this derivative to the test function so that we can pass to the limit $\Delta t \rightarrow 0_{+}$in (4.43). For simplicity, we will study (4.43) with the smooth test function $\varphi \in C_{0}^{2}\left(-T, T ; Y_{b}^{\beta}\right)$.

We now treat the first term of the left side of (4.43). It splits into two parts:

$$
\begin{aligned}
& \int_{0}^{T} \int_{\Omega \times D}\left(1+|\mathbf{q}|^{b}\right) G_{c} \frac{\hat{\psi}^{\Delta t,+}-\hat{\psi}^{\Delta t,-}}{\Delta t} \frac{\varphi}{M^{\beta}} d \mathbf{q} d \mathbf{x} d t \\
= & \int_{0}^{T} \int_{\Omega \times D}\left(1+|\mathbf{q}|^{b}\right) G_{c} \frac{\hat{\psi}^{\Delta t,-}(\mathbf{x}, \mathbf{q}, t)}{M^{\beta}} \frac{\varphi(\mathbf{x}, \mathbf{q}, t-\Delta t)-\varphi(\mathbf{x}, \mathbf{q}, t)}{\Delta t} d \mathbf{q} d \mathbf{x} d t \\
& +\int_{0}^{T} \int_{\Omega \times D}\left(1+|\mathbf{q}|^{b}\right) G_{c} \frac{\hat{\psi}^{\Delta t,+}(\mathbf{x}, \mathbf{q}, t) \varphi(\mathbf{x}, \mathbf{q}, t)-\hat{\psi}^{\Delta t,-}(\mathbf{x}, \mathbf{q}, t) \varphi(\mathbf{x}, \mathbf{q}, t-\Delta t)}{M^{\beta} \Delta t} d \mathbf{q} d \mathbf{x} d t
\end{aligned}
$$

The second term of the right-hand side of (4.63) is equal to

$$
\begin{aligned}
& \int_{t_{N-1}}^{t_{N}} \int_{\Omega \times D}\left(1+|\mathbf{q}|^{b}\right) G_{c} \frac{\hat{\psi}^{N}(\mathbf{x}, \mathbf{q}) \varphi(\mathbf{x}, \mathbf{q}, t)}{M^{\beta} \Delta t} d \mathbf{q} d \mathbf{x} d t \\
& -\int_{t_{-1}}^{t_{0}} \int_{\Omega \times D}\left(1+|\mathbf{q}|^{b}\right) G_{c} \frac{\hat{\psi}^{0}(\mathbf{x}, \mathbf{q}) \varphi(\mathbf{x}, \mathbf{q}, t)}{M^{\beta} \Delta t} d \mathbf{q} d \mathbf{x} d t
\end{aligned}
$$

Then for $\varphi \in C_{0}^{2}\left(-T, T ; Y_{b}^{\beta}\right)$, we see that

$$
\begin{aligned}
& \int_{0}^{T} \int_{\Omega \times D}\left(1+|\mathbf{q}|^{b}\right) G_{c} \frac{\hat{\psi}^{\Delta t,+}(\mathbf{x}, \mathbf{q}, t) \varphi(\mathbf{x}, \mathbf{q}, t)-\hat{\psi}^{\Delta t,-}(\mathbf{x}, \mathbf{q}, t) \varphi(\mathbf{x}, \mathbf{q}, t-\Delta t)}{M^{\beta} \Delta t} d \mathbf{q} d \mathbf{x} d t \\
= & -\int_{t_{-1}}^{t_{0}} \int_{\Omega \times D}\left(1+|\mathbf{q}|^{b}\right) G_{c} \frac{\hat{\psi}^{0}(\mathbf{x}, \mathbf{q}) \varphi(\mathbf{x}, \mathbf{q}, t)}{M^{\beta} \Delta t} d \mathbf{q} d \mathbf{x} d t \\
= & -\int_{\Omega \times D}\left(1+|\mathbf{q}|^{b}\right) G_{c} \frac{\hat{\psi}^{0}(\mathbf{x}, \mathbf{q})}{M^{\beta}}\left(\frac{1}{\Delta t} \int_{0}^{\Delta t} \varphi(\mathbf{x}, \mathbf{q}, t-\Delta t) d t\right) d \mathbf{q} d \mathbf{x} .
\end{aligned}
$$


Further, on recalling the Newton-Leibniz formula, we know that

$$
\begin{aligned}
& \|(\varphi(\mathbf{x}, \mathbf{q}, t-\triangle t)-\varphi(\mathbf{x}, \mathbf{q}, 0)) / \triangle t\|_{L^{2}\left(\Omega \times D ;\left(1+|\mathbf{q}|^{b}\right) M^{-\beta}\right)} \\
= & \left\|\Delta t^{-1} \int_{0}^{t-\Delta t} \frac{\partial \varphi(\mathbf{x}, \mathbf{q}, \tau)}{\partial \tau} d \tau\right\|_{L^{2}\left(\Omega \times D ;\left(1+|\mathbf{q}|^{b}\right) M^{-\beta}\right)} \\
\leq & \max _{[-T, T]}\left\|\frac{\partial \varphi(\mathbf{x}, \mathbf{q}, t)}{\partial t}\right\|_{L^{2}\left(\Omega \times D ;\left(1+|\mathbf{q}|^{b}\right) G_{c} M^{-\beta}\right)} .
\end{aligned}
$$

Thus, application of Lebesgue's dominated convergence theorem and (4.14) yields

$$
\begin{aligned}
& \mid-\int_{\Omega \times D}\left(1+|\mathbf{q}|^{b}\right) G_{c} \frac{\hat{\psi}^{0}(\mathbf{x}, \mathbf{q})}{M^{\beta}}\left(\frac{1}{\Delta t} \int_{0}^{\triangle t} \varphi(\mathbf{x}, \mathbf{q}, t-\triangle t) d t\right) d \mathbf{q} d \mathbf{x} \\
& \quad+\int_{\Omega \times D}\left(1+|\mathbf{q}|^{b}\right) G_{c} \frac{\hat{\psi}^{0}(\mathbf{x}, \mathbf{q})}{M^{\beta}} \varphi(\mathbf{x}, \mathbf{q}, 0) d \mathbf{q} d \mathbf{x} d t \mid \\
& =\left|\int_{\Omega \times D}\left(1+|\mathbf{q}|^{b}\right) G_{c} \frac{\hat{\psi}^{0}(\mathbf{x}, \mathbf{q})}{M^{\beta}}\left(\frac{1}{\Delta t} \int_{0}^{\triangle t}(\varphi(\mathbf{x}, \mathbf{q}, t-\triangle t)-\varphi(\mathbf{x}, \mathbf{q}, 0)) d t\right) d \mathbf{q} d \mathbf{x}\right| \\
& \leq C\left(\frac{\partial \varphi}{\partial t}\right) \triangle t\left\|\left(1+|\mathbf{q}|^{b}\right) G_{c} \frac{\left|\hat{\psi}^{0}(\mathbf{x}, \mathbf{q})\right|^{2}}{M^{\beta}}\right\|_{L^{1}(\Omega \times D)}^{\frac{1}{2}} \rightarrow 0 \quad \text { as } \triangle t \rightarrow 0_{+},
\end{aligned}
$$

which implies that for any given $\varphi \in C_{0}^{2}\left(-T, T ; Y_{b}^{\beta}\right)$,

$$
\begin{aligned}
& \lim _{\Delta t \rightarrow 0_{+}} \int_{\Omega \times D}\left(1+|\mathbf{q}|^{b}\right) G_{c} \frac{\hat{\psi}^{0}(\mathbf{x}, \mathbf{q})}{M^{\beta}}\left(\frac{1}{\Delta t} \int_{0}^{\Delta t} \varphi(\mathbf{x}, \mathbf{q}, t-\Delta t) d t\right) d \mathbf{q} d \mathbf{x} \\
= & \int_{\Omega \times D}\left(1+|\mathbf{q}|^{b}\right) \frac{\psi_{0}(\mathbf{x}, \mathbf{q})}{M^{\frac{\beta}{2}}} \varphi(\mathbf{x}, \mathbf{q}, 0) d \mathbf{q} d \mathbf{x} .
\end{aligned}
$$

Similarly, we can apply Lebesgue's dominated convergence theorem to the first part of the right-hand side of (4.63). Thus, it follows from (4.46) that

$$
\begin{aligned}
& \lim _{\triangle t \rightarrow 0_{+}} \int_{0}^{T} \int_{\Omega \times D}\left(1+|\mathbf{q}|^{b}\right) G_{c} \frac{\hat{\psi}^{\Delta t,-}(\mathbf{x}, \mathbf{q}, t)}{M^{\beta}} \frac{\varphi(\mathbf{x}, \mathbf{q}, t-\Delta t)-\varphi(\mathbf{x}, \mathbf{q}, t)}{\Delta t} d \mathbf{q} d \mathbf{x} d t \\
= & -\int_{0}^{T} \int_{\Omega \times D}\left(1+|\mathbf{q}|^{b}\right) \frac{\hat{\psi}}{M^{\beta}} \frac{\partial \varphi}{\partial t} d \mathbf{q} d \mathbf{x} d t .
\end{aligned}
$$

Therefore, gathering (4.63)-(4.65) together, we obtain for any given $\varphi \in$ $C_{0}^{2}\left(-T, T ; Y_{b}^{\beta}\right)$,

$$
\begin{aligned}
& \lim _{\triangle t \rightarrow 0_{+}} \int_{0}^{T} \int_{\Omega \times D}\left(1+|\mathbf{q}|^{b}\right) G_{c} \frac{\hat{\psi}^{\Delta t,+}-\hat{\psi}^{\Delta t,-}}{\Delta t} \frac{\varphi}{M^{\beta}} d \mathbf{q} d \mathbf{x} d t \\
= & -\int_{0}^{T} \int_{\Omega \times D}\left(1+|\mathbf{q}|^{b}\right) \frac{\hat{\psi}}{M^{\beta}} \frac{\partial \varphi}{\partial t} d \mathbf{q} d \mathbf{x} d t-\int_{\Omega \times D}\left(1+|\mathbf{q}|^{b}\right) \frac{\psi_{0}(\mathbf{x}, \mathbf{q})}{M^{\frac{\beta}{2}}} \varphi(\mathbf{x}, \mathbf{q}, 0) d \mathbf{q} d \mathbf{x} .
\end{aligned}
$$


114 GLOBAL EXISTENCE OF THE REGULARIZED HOOKEAN DUMBBELL MODEL

Similarly to (4.63)-(4.66), it follows from (3.21), (4.14), (4.44) and (4.45) that

$$
\begin{aligned}
& \lim _{\triangle t \rightarrow 0_{+}} \int_{0}^{T} \int_{\Omega \times D}|\mathbf{q}|^{b}\left[A\left(\hat{\mathbf{u}}^{\Delta t,+}\right) \hat{\psi}^{\Delta t,+}-A\left(\hat{\mathbf{u}}^{\Delta t,-}\right) \hat{\psi}^{\Delta t,-}\right] \frac{\varphi}{M^{\beta}} d \mathbf{q} d \mathbf{x} d t \\
= & \lim _{\triangle t \rightarrow 0_{+}} \int_{0}^{T} \int_{\Omega \times D}|\mathbf{q}|^{b} \frac{A\left(\hat{\mathbf{u}}^{\Delta t,-}\right) \hat{\psi}^{\Delta t,-}}{M^{\beta}}[\varphi(\mathbf{x}, \mathbf{q}, t-\triangle t)-\varphi(\mathbf{x}, \mathbf{q}, t)] d \mathbf{q} d \mathbf{x} d t \\
& -\lim _{\triangle t \rightarrow 0_{+}} \int_{\Omega \times D}|\mathbf{q}|^{b} \frac{A\left(\hat{\mathbf{u}}^{0}\right) \hat{\psi}^{0}}{M^{\beta}}\left(\int_{0}^{\triangle t} \varphi(\mathbf{x}, \mathbf{q}, t-\triangle t) d t\right) d \mathbf{q} d \mathbf{x} \\
= & -\lim _{\triangle t \rightarrow 0_{+}} \triangle t \int_{0}^{T} \int_{\Omega \times D}|\mathbf{q}|^{b} \frac{A\left(\hat{\mathbf{u}}^{\Delta t,-}\right) \hat{\psi}^{\Delta t,-}}{M^{\beta}} \frac{\partial \varphi(\mathbf{x}, \mathbf{q}, t)}{\partial t} d \mathbf{q} d \mathbf{x} d t \\
& -\lim _{\triangle t \rightarrow 0_{+}} \triangle t A\left(\hat{\mathbf{u}}^{0}\right) \int_{\Omega \times D}|\mathbf{q}|^{b} \frac{\hat{\psi}^{0}}{M^{\beta}} \varphi(\mathbf{x}, \mathbf{q}, 0) d \mathbf{q} d \mathbf{x} \\
= & 0 .
\end{aligned}
$$

From (4.13), (4.66)-(4.67) and Lemma 4.3 we can verify that $(\hat{\mathbf{u}}, \hat{\psi})$ satisfies $(4.8)$ for any $\varphi \in C_{0}^{2}\left(-T, T ; Y_{b}^{\beta}\right)$ when we pass to the limit $\triangle t \rightarrow 0_{+}$in (4.43). Thus, (4.8) can be verified to be true for $\varphi \in \mathcal{Y}_{b}^{\beta}$ by using (3.21), (4.62) and Assumption $\left(\mathrm{A}_{2}\right)$ and the denseness of $C_{0}^{2}\left(-T, T ; Y_{b}^{\beta}\right)$ in $\mathcal{Y}_{b}^{\beta}$. This completes the proof of Proposition 4.1.

\section{The proofs of main results}

In this section we will show the proofs of our main results given in Section 2. The proof of Theorem 2.1 is divided into the following two lemmas.

Lemma 5.1. Let assumptions $\left(A_{1}\right)-\left(A_{2}\right)$ be fulfilled and $\alpha, \varepsilon \in(0,1]$. Then there exist $\quad \mathbf{u}_{\alpha, \varepsilon, b} \in L^{\infty}\left(0, T ; \mathbf{L}^{2}(\Omega)\right) \cap L^{2}(0, T ; \mathbf{V}) \cap W^{1, \frac{4}{d}}\left(0, T ; \mathbf{V}^{\prime}\right), \quad \psi_{\alpha, \varepsilon, b} \in L^{2}\left(0, T ; X_{b}^{+}\right) \cap$ $L^{\infty}\left(0, T ; L^{2}\left(\Omega \times D ; 1+|\mathbf{q}|^{b}\right)\right)$ and a subsequence $\left\{\mathbf{u}_{\alpha, \beta, \varepsilon, b}, \psi_{\alpha, \beta, \varepsilon, b}\right\}_{\beta}$ satisfying

$$
\begin{aligned}
& \left(1+|\mathbf{q}|^{b}\right)^{\frac{1}{2}} \frac{\psi_{\alpha, \beta, \varepsilon, b}}{M^{\frac{\beta}{2}}} \stackrel{*}{\rightarrow}\left(1+|\mathbf{q}|^{b}\right)^{\frac{1}{2}} \psi_{\alpha, \varepsilon, b} \text { in } L^{\infty}\left(0, T ; L^{2}(\Omega \times D)\right), \\
& \beta \mathbf{q}\left(1+|\mathbf{q}|^{b}\right)^{\frac{1}{2}} \frac{\psi_{\alpha, \beta, \varepsilon, b}}{M^{\frac{\beta}{2}}} \rightarrow \mathbf{0} \text { in } L^{2}\left(0, T ; \mathbf{L}^{2}(\Omega \times D)\right), \\
& \varepsilon\left(1+|\mathbf{q}|^{b}\right)^{\frac{1}{2}} M^{\frac{\beta}{2}} \nabla_{x}\left(\frac{\psi_{\alpha, \beta, \varepsilon, b}}{M^{\beta}}\right)-\varepsilon\left(1+|\mathbf{q}|^{b}\right)^{\frac{1}{2}} \nabla_{x} \psi_{\alpha, \varepsilon, b} \text { in } L^{2}\left(0, T ; \mathbf{L}^{2}(\Omega \times D)\right), \\
& \left(1+|\mathbf{q}|^{b}\right)^{\frac{1}{2}} M^{\frac{\beta}{2}} \nabla_{q}\left(\frac{\psi_{\alpha, \beta, \varepsilon, b}}{M^{\beta}}\right) \rightarrow\left(1+|\mathbf{q}|^{b}\right)^{\frac{1}{2}} \nabla_{q} \psi_{\alpha, \varepsilon, b} \text { in } L^{2}\left(0, T ; \mathbf{L}^{2}(\Omega \times D)\right), \\
& \mathbf{C}\left(\psi_{\alpha, \beta, \varepsilon, b}\right) \stackrel{*}{\rightarrow} \mathbf{C}\left(\psi_{\alpha, \varepsilon, b}\right) \text { in } L^{\infty}\left(0, T ; \mathbf{L}^{2}(\Omega)\right), \\
& \mathbf{u}_{\alpha, \beta, \varepsilon, b} \stackrel{*}{\rightarrow} \mathbf{u}_{\alpha, \varepsilon, b} \text { in } L^{\infty}\left(0, T ; \mathbf{L}^{2}(\Omega)\right), \\
& \mathbf{u}_{\alpha, \beta, \varepsilon, b} \rightarrow \mathbf{u}_{\alpha, \varepsilon, b} \text { in } L^{2}(0, T ; \mathbf{V}) \text {, } \\
& \mathbf{S}_{\gamma} \frac{\partial \mathbf{u}_{\alpha, \beta, \varepsilon, b}}{\partial t} \rightarrow \mathbf{S}_{\gamma} \frac{\partial \mathbf{u}_{\alpha, \varepsilon, b}}{\partial t} \text { in } L^{\frac{4}{d}}(0, T ; \mathbf{V}) \\
& \mathbf{u}_{\alpha, \beta, \varepsilon, b} \rightarrow \mathbf{u}_{\alpha, \varepsilon, b} \text { in } L^{2}\left(0, T ; \mathbf{L}^{r}(\Omega)\right), \\
& \mathbf{J}_{\alpha}^{x} \mathbf{u}_{\alpha, \beta, \varepsilon, b} \rightarrow \mathbf{J}_{\alpha}^{x} \mathbf{u}_{\alpha, \varepsilon, b} \text { in } L^{2}\left(0, T ; \mathbf{W}^{1, \infty}(\Omega)\right) \text {, } \\
& \mathbf{J}_{\alpha}^{x} \mathbf{u}_{\alpha, \beta, \varepsilon, b} \stackrel{*}{\rightarrow} \mathbf{J}_{\alpha}^{x} \mathbf{u}_{\alpha, \varepsilon, b} \text { in } L^{\infty}\left(0, T ; \mathbf{W}^{1, \infty}(\Omega)\right) \text {, }
\end{aligned}
$$

as $\beta \rightarrow 0_{+}$, where $r \in[1, \infty)$ if $d=2$ and $r \in[1,6)$ if $d=3$. 
Proof. Following a similar argument to that which we used in deriving (4.46)(4.47) and (4.50)-(4.56) in Lemma 4.3, we can use (4.57)-(4.58) to obtain (5.1)-(5.2) and $(5.5)-(5.11)$.

It follows from the third term on the left-hand side of (4.57) that (5.4) holds for some limit $\mathbf{g} \in L^{2}\left(0, T ; \mathbf{L}^{2}(\Omega \times D)\right)$. On the other hand, from (3.2) and (5.1), we have $\forall \eta \in L^{2}\left(0, T ; \mathbf{C}_{0}^{\infty}(\Omega \times D)\right)$,

$$
\begin{aligned}
& \int_{0}^{T} \int_{\Omega \times D}\left(1+|\mathbf{q}|^{b}\right)^{\frac{1}{2}} M^{\frac{\beta}{2}} \nabla_{q}\left(\frac{\psi_{\alpha, \beta, \varepsilon, b}}{M^{\beta}}\right) \cdot \eta d \mathbf{q} d \mathbf{x} d t \\
= & \int_{0}^{T} \int_{\Omega \times D} \frac{\psi_{\alpha, \beta, \varepsilon, b}}{M^{\frac{\beta}{2}}}\left[\frac{\beta}{2}\left(1+|\mathbf{q}|^{b}\right)^{\frac{1}{2}} \mathbf{q} \cdot \eta-\left(1+|\mathbf{q}|^{b}\right)^{\frac{1}{2}} \nabla_{q} \cdot \eta-\nabla_{q}\left(1+|\mathbf{q}|^{b}\right)^{\frac{1}{2}} \cdot \eta\right] d \mathbf{q} d \mathbf{x} d t \\
& \rightarrow-\int_{0}^{T} \int_{\Omega \times D} \psi_{\alpha, \varepsilon, b}\left[\left(1+|\mathbf{q}|^{b}\right)^{\frac{1}{2}} \nabla_{q} \cdot \eta+\nabla_{q}\left(1+|\mathbf{q}|^{b}\right)^{\frac{1}{2}} \cdot \eta\right] d \mathbf{q} d \mathbf{x} d t \\
= & -\int_{0}^{T} \int_{\Omega \times D} \psi_{\alpha, \varepsilon, b} \nabla_{q} \cdot\left[\left(1+|\mathbf{q}|^{b}\right)^{\frac{1}{2}} \eta\right] d \mathbf{q} d \mathbf{x} d t \text { as } \beta \rightarrow 0_{+},
\end{aligned}
$$

which shows (5.4) in virtue of the denseness of $\mathbf{C}_{0}^{\infty}(\Omega \times D)$ in $\mathbf{L}^{2}(\Omega \times D)$. In the same way, we can get (5.3) from (4.57). Finally, the non-negativity of $\psi_{\alpha, \beta, \varepsilon, b}$ implies the non-negativity of $\psi_{\alpha, \varepsilon, b}$. The proof is thus complete.

COROLLARY 5.1. It follows from (4.57)-(4.58) and Lemma 5.1 that

$$
\begin{aligned}
& \sup _{t \in(0, T)} {\left[\int_{\Omega \times D}\left(1+|\mathbf{q}|^{b}\right)\left|\psi_{\alpha, \varepsilon, b}\right|^{2} d \mathbf{q} d \mathbf{x}\right]+2 \varepsilon \int_{0}^{T} \int_{\Omega \times D}\left(1+|\mathbf{q}|^{b}\right)\left|\nabla_{x} \psi_{\alpha, \varepsilon, b}\right|^{2} d \mathbf{q} d \mathbf{x} d t } \\
&+\frac{1}{\lambda} \int_{0}^{T} \int_{\Omega \times D}\left(1+|\mathbf{q}|^{b}\right)\left|\nabla_{q} \psi_{\alpha, \varepsilon, b}\right|^{2} d \mathbf{q} d \mathbf{x} d t+\sup _{t \in(0, T)}\left[\int_{\Omega}\left|\mathbf{C}\left(\psi_{\alpha, \varepsilon, b}\right)\right|^{2} d \mathbf{x}\right] \\
&+\int_{0}^{T}\left\|\mathbf{S}_{\gamma}\left(\frac{\partial \mathbf{u}_{\alpha, \varepsilon, b}}{\partial t}\right)\right\|_{H^{1}(\Omega)}^{\frac{4}{d}} d t \leq C(\alpha, T) \\
& \sup _{t \in(0, T)}\left[\int_{\Omega}\left|\mathbf{u}_{\alpha, \varepsilon, b}\right|^{2} d \mathbf{x}\right]+2 \nu \int_{0}^{T} \int_{\Omega}\left|\nabla_{x} \mathbf{u}_{\alpha, \varepsilon, b}\right|^{2} d \mathbf{x} d t \leq C(\alpha)
\end{aligned}
$$

Lemma 5.2. The limit function $\left(\mathbf{u}_{\alpha, \varepsilon, b}, \psi_{\alpha, \varepsilon, b}\right)$ is the weak solution to the regularized Hookean dumbbell model in the sense of Definition 2.1.

Proof. Proceeding similarly as in the proof of Proposition 4.1, we may pass to the limit, $\beta \rightarrow 0_{+}$, in (4.7) to obtain that $\left(\mathbf{u}_{\alpha, \varepsilon, b}, \psi_{\alpha, \varepsilon, b}\right)$ satisfies (2.20) and (2.22), by using (5.5) and (5.7)-(5.9) and on noting (3.20), (3.22), (4.20), (4.62). Then we only need to prove that $\left(\mathbf{u}_{\alpha, \varepsilon, b}, \psi_{\alpha, \varepsilon, b}\right)$ satisfies $(2.21)$.

Now we shall be deal with (4.8) term by term to get $(2.21)$. For $\varphi \in C_{0}^{2}(-T, T ; \mathcal{K})$ fixed. Then $\varphi \in C_{0}^{2}\left(-T, T ; Y_{b}\right)$. Let $\varphi_{\beta}:=M^{\frac{\beta}{2}} \varphi$. Note that $\frac{\varphi_{\beta}}{M^{\frac{\beta}{2}}}=\varphi$, then from (3.2), we have

$$
\begin{aligned}
M^{\beta}\left|\nabla_{q} \frac{\varphi_{\beta}}{M^{\beta}}\right|^{2} & =M^{\beta}\left|M^{-\frac{\beta}{2}} \nabla_{q} \varphi+\varphi \nabla_{q} M^{-\frac{\beta}{2}}\right|^{2} \\
& \leq 2\left|\nabla_{q} \varphi\right|^{2}+\frac{1}{2}|\mathbf{q} \varphi|^{2} .
\end{aligned}
$$


116 GLOBAL EXISTENCE OF THE REGULARIZED HOOKEAN DUMBBELL MODEL

Thus $\varphi_{\beta} \in C_{0}^{2}\left(-T, T ; Y_{b}^{\beta}\right)$ follows immediately. Noting (5.1) and $\varphi \in C_{0}^{2}(-T, T ; \mathcal{K})$, we obtain as $\beta \rightarrow 0_{+}$,

$$
\begin{aligned}
\int_{0}^{T} \int_{\Omega \times D}\left(1+|\mathbf{q}|^{b}\right) \frac{\psi_{\alpha, \beta, \varepsilon, b}}{M^{\beta}} \frac{\partial \varphi_{\beta}}{\partial t} d \mathbf{q} d \mathbf{x} d t & =\int_{0}^{T} \int_{\Omega \times D}\left(1+|\mathbf{q}|^{b}\right) \frac{\psi_{\alpha, \beta, \varepsilon, b}}{M^{\frac{\beta}{2}}} \frac{\partial \varphi}{\partial t} d \mathbf{q} d \mathbf{x} d t \\
& \rightarrow \int_{0}^{T} \int_{\Omega \times D}\left(1+|\mathbf{q}|^{b}\right) \psi_{\alpha, \varepsilon, b} \frac{\partial \varphi}{\partial t} d \mathbf{q} d \mathbf{x} d t
\end{aligned}
$$

It is obvious that

$$
\int_{\Omega \times D}\left(1+|\mathbf{q}|^{b}\right) \frac{\psi_{0}(\cdot, \cdot)}{M^{\frac{\beta}{2}}} \varphi_{\beta}(\cdot, \cdot, 0) d \mathbf{q} d \mathbf{x}=\int_{\Omega \times D}\left(1+|\mathbf{q}|^{b}\right) \psi_{0}(\cdot, \cdot) \varphi(\cdot, \cdot, 0) d \mathbf{q} d \mathbf{x} .
$$

Using Lebesgue's dominated convergence theorem, and noting (3.2), (5.4) and $\psi_{\alpha, \beta, \varepsilon, b} \in L^{2}\left(0, T ; Z_{b}^{\beta}\right)$, we obtain that for any $\varphi \in C_{0}^{2}(-T, T ; \mathcal{K})$,

$$
\begin{aligned}
& \lim _{\beta \rightarrow 0_{+}} \int_{0}^{T} \int_{\Omega \times D}\left(M^{\beta} \nabla_{q} \frac{\psi_{\alpha, \beta, \varepsilon, b}}{M^{\beta}}\right) \cdot\left[\nabla_{q}\left(\left(1+|\mathbf{q}|^{b}\right) \frac{\varphi_{\beta}}{M^{\beta}}\right)-\nabla_{q}\left(\left(1+|\mathbf{q}|^{b}\right) \varphi\right)\right] d \mathbf{q} d \mathbf{x} d t \\
= & \lim _{\beta \rightarrow 0_{+}} \int_{0}^{T} \int_{\Omega \times D}\left(M^{\beta} \nabla_{q} \frac{\psi_{\alpha, \beta, \varepsilon, b}}{M^{\beta}}\right) \cdot \nabla_{q}\left[\left(1+|\mathbf{q}|^{b}\right)\left(\frac{\varphi}{M^{\frac{\beta}{2}}}-\varphi\right)\right] d \mathbf{q} d \mathbf{x} d t \\
= & \lim _{\beta \rightarrow 0_{+}} \int_{0}^{T} \int_{\Omega \times D}\left(M^{\beta} \nabla_{q} \frac{\psi_{\alpha, \beta, \varepsilon, b}}{M^{\beta}}\right) \cdot\left[\left(1+|\mathbf{q}|^{b}\right) \nabla_{q}\left(\frac{\varphi}{M^{\frac{\beta}{2}}}-\varphi\right)\right] d \mathbf{q} d \mathbf{x} d t \\
= & \lim _{\beta \rightarrow 0_{+}} \int_{0}^{T} \int_{\Omega \times D}\left(M^{\beta} \nabla_{q} \frac{\psi_{\alpha, \beta, \varepsilon, b}}{M^{\beta}}\right) \cdot\left(1+|\mathbf{q}|^{b}\right)\left[\nabla_{q} \varphi\left(M^{-\frac{\beta}{2}}-1\right)+\frac{\beta}{2} \mathbf{q} M^{-\frac{\beta}{2}} \varphi\right] d \mathbf{q} d \mathbf{x} d t \\
= & 0 .
\end{aligned}
$$

This together with (5.4) shows that

$$
\begin{aligned}
& \lim _{\beta \rightarrow 0_{+}} \int_{0}^{T} \int_{\Omega \times D}\left(M^{\beta} \nabla_{q} \frac{\psi_{\alpha, \beta, \varepsilon, b}}{M^{\beta}}\right) \cdot \nabla_{q}\left(\left(1+|\mathbf{q}|^{b}\right) \frac{\varphi_{\beta}}{M^{\beta}}\right) d \mathbf{q} d \mathbf{x} d t \\
= & \lim _{\beta \rightarrow 0_{+}} \int_{0}^{T} \int_{\Omega \times D}\left(M^{\beta} \nabla_{q} \frac{\psi_{\alpha, \beta, \varepsilon, b}}{M^{\beta}}\right) \cdot \nabla_{q}\left(\left(1+|\mathbf{q}|^{b}\right) \varphi\right) d \mathbf{q} d \mathbf{x} d t \\
= & \int_{0}^{T} \int_{\Omega \times D} \nabla_{q} \psi_{\alpha, \varepsilon, b} \cdot \nabla_{q}\left(\left(1+|\mathbf{q}|^{b}\right) \varphi\right) d \mathbf{q} d \mathbf{x} d t .
\end{aligned}
$$

By (3.2) and (5.2) we have

$$
\begin{aligned}
& \mid \int_{0}^{T} \int_{\Omega \times D}\left[\nabla_{x}\left(\mathbf{J}_{\alpha}^{x} \mathbf{u}_{\alpha, \beta, \varepsilon, b}\right) \mathbf{q}\right] \frac{\psi_{\alpha, \beta, \varepsilon, b}}{M^{\frac{\beta}{2}}} \cdot\left(M^{\frac{\beta}{2}} \nabla_{q}\left(\left(1+|\mathbf{q}|^{b}\right) \frac{\varphi_{\beta}}{M^{\beta}}\right)\right. \\
& \left.-\nabla_{q}\left(\left(1+|\mathbf{q}|^{b}\right) \varphi\right)\right) d \mathbf{q} d \mathbf{x} d t \mid \\
& =\left|\int_{0}^{T} \int_{\Omega \times D}\left[\nabla_{x}\left(\mathbf{J}_{\alpha}^{x} \mathbf{u}_{\alpha, \beta, \varepsilon, b}\right) \mathbf{q}\right] \frac{\psi_{\alpha, \beta, \varepsilon, b}}{M^{\frac{\beta}{2}}} \cdot\left(M^{\frac{\beta}{2}} \nabla_{q} \frac{\varphi}{M^{\frac{\beta}{2}}}-\nabla_{q} \varphi\right)\left(1+|\mathbf{q}|^{b}\right) d \mathbf{q} d \mathbf{x} d t\right| \\
& =\left|\int_{0}^{T} \int_{\Omega \times D} \frac{\beta}{2}\left[\nabla_{x}\left(\mathbf{J}_{\alpha}^{x} \mathbf{u}_{\alpha, \beta, \varepsilon, b}\right) \mathbf{q}\right] \frac{\psi_{\alpha, \beta, \varepsilon, b}}{M^{\frac{\beta}{2}}} \cdot\left(\mathbf{q}\left(1+|\mathbf{q}|^{b}\right) \varphi\right) d \mathbf{q} d \mathbf{x} d t\right| \rightarrow 0, \quad \text { as } \beta \rightarrow 0_{+} .
\end{aligned}
$$


Note that $\mathbf{J}_{\alpha}^{x} \mathbf{u}_{\alpha, \varepsilon, b} \in L^{\infty}\left(0, T ; W^{1, \infty}(\Omega)\right)$ from (5.11). Then by using (5.1) and (5.10), we obtain

$$
\begin{aligned}
& \mid \int_{0}^{T} \int_{\Omega \times D}\left(\left[\nabla_{x}\left(\mathbf{J}_{\alpha}^{x} \mathbf{u}_{\alpha, \beta, \varepsilon, b}\right) \mathbf{q}\right] \frac{\psi_{\alpha, \beta, \varepsilon, b}}{M^{\frac{\beta}{2}}}-\left[\nabla_{x}\left(\mathbf{J}_{\alpha}^{x} \mathbf{u}_{\alpha, \varepsilon, b}\right) \mathbf{q}\right] \psi_{\alpha, \varepsilon, b}\right) \\
& \cdot \nabla_{q}\left(\left(1+|\mathbf{q}|^{b}\right) \varphi\right) d \mathbf{q} d \mathbf{x} d t \mid \\
& \leq\left|\int_{0}^{T} \int_{\Omega \times D}\left[\nabla_{x}\left(\mathbf{J}_{\alpha}^{x} \mathbf{u}_{\alpha, \beta, \varepsilon, b}\right) \mathbf{q}-\nabla_{x}\left(\mathbf{J}_{\alpha}^{x} \mathbf{u}_{\alpha, \varepsilon, b}\right) \mathbf{q}\right] \frac{\psi_{\alpha, \beta, \varepsilon, b}}{M^{\frac{\beta}{2}}} \cdot \nabla_{q}\left(\left(1+|\mathbf{q}|^{b}\right) \varphi\right) d \mathbf{q} d \mathbf{x} d t\right| \\
& \quad+\left|\int_{0}^{T} \int_{\Omega \times D}\left[\nabla_{x}\left(\mathbf{J}_{\alpha}^{x} \mathbf{u}_{\alpha, \varepsilon, b}\right) \mathbf{q}\right]\left(\frac{\psi_{\alpha, \beta, \varepsilon, b}}{M^{\frac{\beta}{2}}}-\psi_{\alpha, \varepsilon, b}\right) \cdot \nabla_{q}\left(\left(1+|\mathbf{q}|^{b}\right) \varphi\right) d \mathbf{q} d \mathbf{x} d t\right| \\
& \rightarrow 0 \text { as } \beta \rightarrow 0_{+} .
\end{aligned}
$$

Combination of the above two relations leads to

$$
\begin{aligned}
& \lim _{\beta \rightarrow 0_{+}} \int_{0}^{T} \int_{\Omega \times D}\left[\nabla_{x}\left(\mathbf{J}_{\alpha}^{x} \mathbf{u}_{\alpha, \beta, \varepsilon, b}\right) \mathbf{q}\right] \psi_{\alpha, \beta, \varepsilon, b} \cdot \nabla_{q}\left(\left(1+|\mathbf{q}|^{b}\right) \frac{\varphi_{\beta}}{M^{\beta}}\right) d \mathbf{q} d \mathbf{x} d t \\
= & \int_{0}^{T} \int_{\Omega \times D}\left[\nabla_{x}\left(\mathbf{J}_{\alpha}^{x} \mathbf{u}_{\alpha, \varepsilon, b}\right) \mathbf{q}\right] \psi_{\alpha, \varepsilon, b} \cdot \nabla_{q}\left(\left(1+|\mathbf{q}|^{b}\right) \varphi\right) d \mathbf{q} d \mathbf{x} d t .
\end{aligned}
$$

By (3.2) and (5.2), we further have

$$
\begin{aligned}
& \int_{0}^{T} \int_{\Omega \times D} \mathbf{q} \psi_{\alpha, \beta, \varepsilon, b} \cdot \nabla_{q}\left(\left(1+|\mathbf{q}|^{b}\right) \frac{\varphi_{\beta}}{M^{\beta}}\right) d \mathbf{q} d \mathbf{x} d t \\
& -\int_{0}^{T} \int_{\Omega \times D} \mathbf{q} \frac{\psi_{\alpha, \beta, \varepsilon, b}}{M^{\frac{\beta}{2}}} \cdot \nabla_{q}\left(\left(1+|\mathbf{q}|^{b}\right) \varphi\right) d \mathbf{q} d \mathbf{x} d t \\
& =\int_{0}^{T} \int_{\Omega \times D}\left(1+|\mathbf{q}|^{b}\right) \mathbf{q} \frac{\psi_{\alpha, \beta, \varepsilon, b}}{M^{\frac{\beta}{2}}} \cdot\left[M^{\frac{\beta}{2}} \nabla_{q} \frac{\varphi}{M^{\frac{\beta}{2}}}-\nabla_{q} \varphi\right] d \mathbf{q} d \mathbf{x} d t \\
& =\frac{\beta}{2} \int_{0}^{T} \int_{\Omega \times D}\left(1+|\mathbf{q}|^{b}\right)|\mathbf{q}|^{2} \frac{\psi_{\alpha, \beta, \varepsilon, b}}{M^{\frac{\beta}{2}}} \varphi d \mathbf{q} d \mathbf{x} d t \rightarrow 0 \quad \text { as } \beta \rightarrow 0_{+} .
\end{aligned}
$$

From $\varphi \in C_{0}^{2}(-T, T ; \mathcal{K})$ and $(5.1)$, the above relation implies that

$$
\begin{aligned}
& \lim _{\beta \rightarrow 0_{+}} \int_{0}^{T} \int_{\Omega \times D} \mathbf{q} \psi_{\alpha, \beta, \varepsilon, b} \cdot \nabla_{q}\left(\left(1+|\mathbf{q}|^{b}\right) \frac{\varphi_{\beta}}{M^{\beta}}\right) d \mathbf{q} d \mathbf{x} d t \\
= & \lim _{\beta \rightarrow 0_{+}} \int_{0}^{T} \int_{\Omega \times D} \mathbf{q} \frac{\psi_{\alpha, \beta, \varepsilon, b}}{M^{\frac{\beta}{2}}} \cdot \nabla_{q}\left(\left(1+|\mathbf{q}|^{b}\right) \varphi\right) d \mathbf{q} d \mathbf{x} d t \\
= & \int_{0}^{T} \int_{\Omega \times D} \mathbf{q} \psi_{\alpha, \varepsilon, b} \cdot \nabla_{q}\left(\left(1+|\mathbf{q}|^{b}\right) \varphi\right) d \mathbf{q} d \mathbf{x} d t .
\end{aligned}
$$


118 GLOBAL EXISTENCE OF THE REGULARIZED HOOKEAN DUMBBELL MODEL

From (5.3), we have

$$
\begin{aligned}
& \lim _{\beta \rightarrow 0_{+}} \int_{0}^{T} \int_{\Omega \times D}\left(1+|\mathbf{q}|^{b}\right)\left(M^{\beta} \nabla_{x} \frac{\psi_{\alpha, \beta, \varepsilon, b}}{M^{\beta}}\right) \cdot \nabla_{x} \frac{\varphi_{\beta}}{M^{\beta}} d \mathbf{q} d \mathbf{x} d t \\
= & \lim _{\beta \rightarrow 0_{+}} \int_{0}^{T} \int_{\Omega \times D}\left(1+|\mathbf{q}|^{b}\right)\left(M^{\frac{\beta}{2}} \nabla_{x} \frac{\psi_{\alpha, \beta, \varepsilon, b}}{M^{\beta}}\right) \cdot \nabla_{x} \varphi d \mathbf{q} d \mathbf{x} d t \\
= & \int_{0}^{T} \int_{\Omega \times D} \nabla_{x} \psi_{\alpha, \varepsilon, b} \cdot \nabla_{x} \varphi d \mathbf{q} d \mathbf{x} d t .
\end{aligned}
$$

Note that

$$
\begin{aligned}
& \int_{0}^{T} \int_{\Omega \times D}\left(1+|\mathbf{q}|^{b}\right)\left(\left[\mathbf{u}_{\alpha, \beta, \varepsilon, b} \psi_{\alpha, \beta, \varepsilon, b}\right] \cdot \nabla_{x} \frac{\varphi_{\beta}}{M^{\beta}}-\left[\mathbf{u}_{\alpha, \varepsilon, b} \psi_{\alpha, \varepsilon, b}\right] \cdot \nabla_{x} \varphi\right) d \mathbf{q} d \mathbf{x} d t \\
= & \int_{0}^{T} \int_{\Omega \times D}\left(1+|\mathbf{q}|^{b}\right)\left(\mathbf{u}_{\alpha, \beta, \varepsilon, b} \frac{\psi_{\alpha, \beta, \varepsilon, b}}{M^{\frac{\beta}{2}}}-\mathbf{u}_{\alpha, \varepsilon, b} \psi_{\alpha, \varepsilon, b}\right) \cdot \nabla_{x} \varphi d \mathbf{q} d \mathbf{x} d t \\
= & \int_{0}^{T} \int_{\Omega \times D}\left(1+|\mathbf{q}|^{b}\right)\left[\left(\mathbf{u}_{\alpha, \beta, \varepsilon, b}-\mathbf{u}_{\alpha, \varepsilon, b}\right) \frac{\psi_{\alpha, \beta, \varepsilon, b}}{M^{\frac{\beta}{2}}}\right] \cdot \nabla_{x} \varphi d \mathbf{q} d \mathbf{x} d t \\
& +\int_{0}^{T} \int_{\Omega \times D}\left(1+|\mathbf{q}|^{b}\right) \mathbf{u}_{\alpha, \varepsilon, b}\left(\frac{\psi_{\alpha, \beta, \varepsilon, b}}{M^{\frac{\beta}{2}}}-\psi_{\alpha, \varepsilon, b}\right) \cdot \nabla_{x} \varphi d \mathbf{q} d \mathbf{x} d t .
\end{aligned}
$$

Then it is obvious that

$$
\begin{aligned}
& \lim _{\beta \rightarrow 0_{+}} \int_{0}^{T} \int_{\Omega \times D}\left(1+|\mathbf{q}|^{b}\right)\left[\mathbf{u}_{\alpha, \beta, \varepsilon, b} \psi_{\alpha, \beta, \varepsilon, b}\right] \cdot \nabla_{x} \frac{\varphi_{\beta}}{M^{\beta}} d \mathbf{q} d \mathbf{x} d t \\
= & \int_{0}^{T} \int_{\Omega \times D}\left(1+|\mathbf{q}|^{b}\right) \mathbf{u}_{\alpha, \varepsilon, b} \psi_{\alpha, \varepsilon, b} \cdot \nabla_{x} \varphi d \mathbf{q} d \mathbf{x} d t .
\end{aligned}
$$

Noticing $\mathbf{J}_{\alpha}^{x} \mathbf{u}_{\alpha, \varepsilon, b} \in L^{\infty}\left(0, T ; W^{1, \infty}(\Omega)\right)$, from (4.57) we have

$$
\begin{aligned}
& \left.\quad \beta\left|\int_{\Omega \times D} A\left(\mathbf{u}_{\alpha, \beta, \varepsilon}\right)\right| \mathbf{q}\right|^{2}\left(1+|\mathbf{q}|^{b}\right) \frac{\psi_{\alpha, \beta, \varepsilon, b}}{M^{\beta}} \varphi_{\beta} d \mathbf{q} d \mathbf{x} d t \mid \\
& \leq C \beta^{\frac{1}{2}} \rightarrow 0 \quad \text { as } \beta \rightarrow 0_{+} .
\end{aligned}
$$

At last, gathering (5.14)-(5.21) together yields that $\left(\mathbf{u}_{\alpha, \varepsilon, b}, \psi_{\alpha, \varepsilon, b}\right)$ satisfies

$$
\begin{aligned}
& -\int_{0}^{T} \int_{\Omega \times D}\left(1+|\mathbf{q}|^{b}\right) \psi_{\alpha, \varepsilon, b} \frac{\partial \varphi}{\partial t} d \mathbf{q} d \mathbf{x} d t-\int_{\Omega \times D}\left(1+|\mathbf{q}|^{b}\right) \psi_{0}(\cdot, \cdot) \varphi(\cdot, \cdot, 0) d \mathbf{q} d \mathbf{x} \\
& \quad+\int_{0}^{T} \int_{\Omega \times D}\left[\frac{1}{2 \lambda} \nabla_{q} \psi_{\alpha, \varepsilon, b}-\left[\nabla_{x}\left(\mathbf{J}_{\alpha}^{x} \mathbf{u}_{\alpha, \varepsilon, b}\right) \mathbf{q}\right] \psi_{\alpha, \varepsilon, b}+\frac{1}{2 \lambda} \mathbf{q} \psi_{\alpha, \varepsilon, b}\right] \\
& \cdot \nabla_{q}\left(\left(1+|\mathbf{q}|^{b}\right) \varphi\right) d \mathbf{q} d \mathbf{x} d t \\
& \quad+\int_{0}^{T} \int_{\Omega \times D}\left(1+|\mathbf{q}|^{b}\right)\left[\varepsilon \nabla_{x} \psi_{\alpha, \varepsilon, b}-\mathbf{u}_{\alpha, \varepsilon, b} \psi_{\alpha, \varepsilon, b}\right] \cdot \nabla_{x} \varphi d \mathbf{q} d \mathbf{x} d t=0, \\
& \forall \varphi \in C_{0}^{2}(-T, T ; \mathcal{K}) .
\end{aligned}
$$

Thus, it is easy to deduce that the above equation remains true for any $\varphi \in \mathcal{Y}_{b}$ in virtue of $(4.62)$ and the density of $C_{0}^{2}(-T, T ; \mathcal{K})$ in $\mathcal{Y}_{b}$. The proof is completed. 
Following the same procedure developed earlier in this section, we conclude Theorem 2.2 but omit its proof. Moreover, a corollary follows immediately from (5.12)(5.13) and Theorem 2.2.

Corollary 5.2.

$$
\begin{aligned}
\sup _{t \in(0, T)} & {\left[\int_{\Omega \times D}\left(1+|\mathbf{q}|^{b}\right)\left|\psi_{\alpha, b}\right|^{2} d \mathbf{q} d \mathbf{x}\right]+\frac{1}{\lambda} \int_{0}^{T} \int_{\Omega \times D}\left(1+|\mathbf{q}|^{b}\right)\left|\nabla_{q} \psi_{\alpha, b}\right|^{2} d \mathbf{q} d \mathbf{x} d t } \\
& +\sup _{t \in(0, T)}\left[\int_{\Omega}\left|\mathbf{C}\left(\psi_{\alpha, b}\right)\right|^{2} d \mathbf{x}\right]+\int_{0}^{T}\left\|\mathbf{S}_{\gamma}\left(\frac{\partial \mathbf{u}_{\alpha, b}}{\partial t}\right)\right\|_{H^{1}(\Omega)}^{\frac{4}{d}} d t \leq C(\alpha, T), \\
\sup _{t \in(0, T)} & {\left[\int_{\Omega}\left|\mathbf{u}_{\alpha, b}\right|^{2} d \mathbf{x}\right]+2 \nu \int_{0}^{T} \int_{\Omega}\left|\nabla_{x} \mathbf{u}_{\alpha, b}\right|^{2} d \mathbf{x} d t \leq C(\alpha) . }
\end{aligned}
$$

Proof of Proposition 2.3. By using the Hölder inequality and the Cauchy inequality, we have

$$
\begin{aligned}
\int_{\Omega \times D} \psi_{\alpha, b} U d \mathbf{q} d \mathbf{x} & =\frac{1}{2} \int_{\Omega \times D} \psi_{\alpha, b}|\mathbf{q}|^{2} d \mathbf{q} d \mathbf{x} \\
& \leq \frac{|\Omega|^{\frac{1}{2}}}{2}\left[\int_{\Omega}\left(\int_{D} \psi_{\alpha, b}|\mathbf{q}|^{2} d \mathbf{q}\right)^{2} d \mathbf{x}\right]^{\frac{1}{2}} \\
& \leq \frac{\sqrt{d}}{2}|\Omega|^{\frac{1}{2}}\left[\sum_{i=1}^{d} \int_{\Omega}\left(\int_{D} \psi_{\alpha, b}\left|q_{i}\right|^{2} d \mathbf{q}\right)^{2} d \mathbf{x}\right]^{\frac{1}{2}} \\
& \leq \frac{\sqrt{d}}{2}|\Omega|^{\frac{1}{2}}\left\|\mathbf{C}\left(\psi_{\alpha, b}\right)\right\|_{L^{\infty}\left(0, T ; \mathbf{L}^{2}(\Omega)\right)} .
\end{aligned}
$$

Thus $\psi_{\alpha, b} \in L^{\infty}\left(0, T ; L^{1}(\Omega \times D ; U)\right)$. Similarly, it follows from $\mathbf{C}\left(\psi_{\alpha, \varepsilon, b}\right) \in$ $L^{\infty}\left(0, T ; \mathbf{L}^{2}(\Omega)\right)$ that $\psi_{\alpha, \varepsilon, b} \in L^{\infty}\left(0, T ; L^{1}(\Omega \times D ; U)\right)$.

Next, we will prove $(2.26)$. For any $s \in(0, T)$ and $\triangle t$ sufficiently small such that $0<\Delta t<s$, we can choose

$$
\varphi(\mathbf{x}, \mathbf{q}, t)=\frac{[s-t]_{+}-[s-\Delta t-t]_{+}}{\triangle t} \frac{(M(\mathbf{q}))^{\beta}}{1+|\mathbf{q}|^{b}}
$$

in (4.8) to obtain that

$$
\begin{aligned}
& \frac{1}{\triangle t} \int_{s-\triangle t}^{s} \int_{\Omega \times D} \psi_{\alpha, \beta, \varepsilon, b}(\mathbf{x}, \mathbf{q}, t) d \mathbf{q} d \mathbf{x} d t \\
& \quad+\frac{\beta}{2} \int_{0}^{s-\triangle t} \int_{\Omega \times D} A\left(\mathbf{u}_{\alpha, \beta, \varepsilon, b}(\mathbf{x}, \mathbf{q}, t)\right)|\mathbf{q}|^{2} \psi_{\alpha, \beta, \varepsilon, b}(\mathbf{x}, \mathbf{q}, t) d \mathbf{q} d \mathbf{x} d t \\
& \quad+\frac{\beta}{2} \frac{1}{\triangle t} \int_{s-\triangle t}^{s} \int_{\Omega \times D}(s-t) A\left(\mathbf{u}_{\alpha, \beta, \varepsilon, b}(\mathbf{x}, \mathbf{q}, t)\right)|\mathbf{q}|^{2} \psi_{\alpha, \beta, \varepsilon, b}(\mathbf{x}, \mathbf{q}, t) d \mathbf{q} d \mathbf{x} d t \\
& =\int_{\Omega \times D} \psi_{0}(\mathbf{x}, \mathbf{q})(M(\mathbf{q}))^{\frac{\beta}{2}} d \mathbf{q} d \mathbf{x} .
\end{aligned}
$$

Letting $\triangle t \rightarrow 0_{+}$, then $\beta \rightarrow 0_{+}$, we have

$$
\int_{\Omega \times D} \psi_{\alpha, \varepsilon, b}(\mathbf{x}, \mathbf{q}, s) d \mathbf{q} d \mathbf{x}=\int_{\Omega \times D} \psi_{0}(\mathbf{x}, \mathbf{q}) d \mathbf{q} d \mathbf{x} \quad \forall s \in(0, T) .
$$


Similarly, we can pass to the limit $\varepsilon \rightarrow 0_{+}$to obtain that

$$
\int_{\Omega \times D} \psi_{\alpha, b}(\mathbf{x}, \mathbf{q}, s) d \mathbf{q} d \mathbf{x}=\int_{\Omega \times D} \psi_{0}(\mathbf{x}, \mathbf{q}) d \mathbf{q} d \mathbf{x} \quad \forall s \in(0, T) .
$$

REMARK 5.3. Note that the existence of the term $(M(\mathbf{q}))^{\beta}$ in the expression of $\varphi(\mathbf{x}, \mathbf{q}, t)$ in (5.24). From (3.1), there exist positive constant $c_{i}, i=1,2$, such that

$$
c_{1} e^{-\frac{1}{2}|\mathbf{q}|^{2}} \leq M(\mathbf{q}) \leq c_{2} e^{-\frac{1}{2}|\mathbf{q}|^{2}} .
$$

Moreover, the gamma function $\Gamma(a):=\int_{0}^{\infty} x^{a-1} e^{-x} d x$ is well defined in the interval $(0, \infty)$. Then, by careful calculation, we can find $\varphi(\mathbf{x}, \mathbf{q}, t) \in \mathcal{Y}_{b}^{\beta}$ in (5.24).

\section{Conclusion}

The global existence of weak solutions to the regularized Hookean dumbbell model is derived in this paper. This regularized model is put forward in [2] which possesses two noteworthy features. One is the presence of a diffusion term $\varepsilon \triangle_{x} \psi$. The other is Friedrichs mollifiers with a parameter $\alpha$. The key techniques of our analysis are to introduce parameters $\beta$ and $b$ and to build a modified model which is treated in the framework of the Rothe method. Then on passage to the limit $\beta \rightarrow 0_{+}$, the regularized Hookean dumbbell model is justified. Moreover, we derive the global existence of weak solutions to the reduced Hookean dumbbell model with $\varepsilon=0$ by passing to the limit $\varepsilon \rightarrow 0_{+}$. We have not yet completed extending our approach to the reduced model with $\alpha=0$ and the classical model with $(\alpha, \varepsilon)=(0,0)$. In a forthcoming paper, we shall attempt to research these models.

Appendix. A. In this section the notation (4.3) is also used and the proofs of Lemma 4.1 and 4.3 are given as follows.

The proof of Lemma 4.1. For any $\hat{\mathbf{v}} \in \mathbf{Y}^{r}$, let $\tilde{\mathbf{v}}:=\mathbf{G}(\hat{\mathbf{v}})$. Then by the definition of $\mathbf{G}$, there exists $\tilde{\phi} \in Y_{b}^{\beta}$ such that $\hat{\mathbf{v}}, \tilde{\mathbf{v}}, \tilde{\phi}$ satisfy $(4.25)-(4.26)$. By choosing $\mathbf{w}=\tilde{\mathbf{v}}$ in (4.26), and noticing (3.20), (3.27), (4.20), we have

$$
\begin{aligned}
& \int_{\Omega}\left[|\tilde{\mathbf{v}}|^{2}+\left|\tilde{\mathbf{v}}-\hat{\mathbf{u}}^{n-1}\right|^{2}-\left|\mathbf{u}^{n-1}\right|^{2}\right] d \mathbf{x}+\Delta t \nu \int_{\Omega}\left|\nabla_{x} \tilde{\mathbf{v}}\right|^{2} d \mathbf{x} \\
\leq & C(\beta) \Delta t \int_{\Omega \times D} \frac{|\tilde{\phi}|^{2}}{M^{\beta}} d \mathbf{q} d \mathbf{x} .
\end{aligned}
$$

By choosing $\varphi=\tilde{\phi}$ in (4.25), and noticing (4.24), we obtain

$$
\begin{aligned}
& \left(1-\frac{2 b(b-1+d)+d}{2 \lambda} \Delta t\right) \int_{\Omega \times D}\left(1+|\mathbf{q}|^{b}\right) G_{c} \frac{|\tilde{\phi}|^{2}}{M^{\beta}} d \mathbf{q} d \mathbf{x} \\
& \quad+2 \Delta t \int_{\Omega \times D}\left(1+|\mathbf{q}|^{b}\right)\left(\frac{W_{c}}{2 \lambda} M^{\beta}\left|\nabla_{q} \frac{\tilde{\phi}}{M^{\beta}}\right|^{2}+\varepsilon M^{\beta}\left|\nabla_{x} \frac{\tilde{\phi}}{M^{\beta}}\right|^{2}\right) d \mathbf{q} d \mathbf{x} \\
& \quad+\frac{b(1-2 \beta)}{4 \lambda} \Delta t \int_{\Omega \times D}|\mathbf{q}|^{b} \frac{|\tilde{\phi}|^{2}}{M^{\beta}} d \mathbf{q} d \mathbf{x}+\frac{b \beta \Delta t}{2 \lambda} \int_{\Omega \times D} W_{c}|\mathbf{q}|^{b} \frac{|\tilde{\phi}|^{2}}{M^{\beta}} d \mathbf{q} d \mathbf{x} \\
& \quad+\frac{\beta(1-\beta)}{2 \lambda} \Delta t \int_{\Omega \times D}\left(1+|\mathbf{q}|^{b}\right)|\mathbf{q}|^{2} \frac{|\tilde{\phi}|^{2}}{M^{\beta}} d \mathbf{q} d \mathbf{x} \\
& \leq \int_{\Omega \times D}\left(1+|\mathbf{q}|^{b}\right) G_{c} \frac{\left|\hat{\psi}^{n-1}\right|^{2}}{M^{\beta}} d \mathbf{q} d \mathbf{x}+b \lambda\left(A\left(\hat{\mathbf{u}}^{n-1}\right)\right)^{2} \triangle t \int_{\Omega \times D}|\mathbf{q}|^{b} \frac{\left|\hat{\psi}^{n-1}\right|^{2}}{M^{\beta}} d \mathbf{q} d \mathbf{x} \\
& \leq C(\alpha) .
\end{aligned}
$$


Let $\triangle t<\min \left(1, \frac{2 \lambda}{2 b(b-1+d)+d}\right)$. Combining (A.1) and (A.2) and applying the Sobolev imbedding $\mathbf{V} \hookrightarrow \mathbf{L}^{r}(\Omega)$ and the Poincaré inequality, we deduce that

$$
\|\tilde{\mathbf{v}}\|_{L^{r}(\Omega)} \leq C\left\|\nabla_{x} \tilde{\mathbf{v}}\right\|_{L^{2}(\Omega)} \leq C^{*}(\alpha, \beta) \text { for } r \in(d, 6) .
$$

Thus we see that $G$ is a mapping from $\mathbf{E}:=\left\{\mathbf{w} \in \mathbf{Y}^{r}:\|\mathbf{w}\|_{L^{r}(\Omega)} \leq C^{*}(\alpha, \beta)\right\} \subset \mathbf{Y}^{r}$ into E.

Next, we will show that $\mathbf{G}$ is continuous and compact. Therefore, by Schauder's fixed point theorem, we conclude that $\mathbf{G}$ has a fixed point in $\mathbf{Y}^{r}$.

Suppose any sequence $\left\{\hat{\mathbf{v}}^{(i)}\right\}_{i \geq 0}$ in $\mathbf{Y}^{r}$ such that

$$
\lim _{i \rightarrow \infty}\left\|\hat{\mathbf{v}}^{(i)}-\hat{\mathbf{v}}\right\|_{L^{r}(\Omega)}=0 .
$$

From (3.21) we have

$$
\mathbf{J}_{\alpha}^{x} \hat{\mathbf{v}}^{(i)} \rightarrow \mathbf{J}_{\alpha}^{x} \hat{\mathbf{v}} \text { in } \mathbf{W}^{1, \infty}(\Omega) \text { as } i \rightarrow \infty .
$$

Set $\tilde{\mathbf{v}}^{(i)}:=\mathbf{G}\left(\hat{\mathbf{v}}^{(i)}\right)$. Then there exists $\tilde{\phi}^{(i)} \in Y_{b}^{\beta}$ such that

$$
\begin{aligned}
& a\left(\hat{\mathbf{v}}^{(i)}\right)\left(\tilde{\phi}^{(i)}, \varphi\right)=\int_{\Omega \times D}\left[\left(1+|\mathbf{q}|^{b}\right) G_{c}+\Delta t \frac{b}{2}|\mathbf{q}|^{b} A\left(\mathbf{u}^{n-1}\right)\right] \frac{\hat{\psi}^{n-1} \varphi}{M^{\beta}} d \mathbf{q} d \mathbf{x}, \forall \varphi \in Y_{b}^{\beta} \\
& b\left(\hat{\mathbf{u}}^{n-1}\right)\left(\tilde{\mathbf{v}}^{(i)}, \mathbf{w}\right)=\int_{\Omega}\left[\hat{\mathbf{u}}^{n-1} \cdot \mathbf{w}-\Delta t \kappa \omega \mathbf{C}\left(\tilde{\phi}^{(i)}\right): \nabla_{x}\left(\mathbf{w}_{\alpha}\right)\right] d \mathbf{x} \quad \forall \mathbf{w} \in \mathbf{V}
\end{aligned}
$$

By an argument similar to the above, we can construct the estimates (A.1)-(A.3) for $\tilde{\mathbf{v}}^{(i)}$ and $\tilde{\phi}^{(i)}$. These estimates together with (3.27) and the compact imbedding results of Sobolev spaces $\mathbf{H}^{1}(\Omega) \hookrightarrow \mathbf{L}^{r}(\Omega), r \in(d, 6)$ yield that there exist a subsequence $\left\{\tilde{\phi}^{\left(i_{k}\right)}, \tilde{\mathbf{v}}^{\left(i_{k}\right)}\right\}_{i_{k} \geq 0}$ and functions $\tilde{\phi} \in Y_{b}^{\beta}$ and $\tilde{\mathbf{v}} \in \mathbf{V}$ such that as $i_{k} \rightarrow \infty$,

$$
\begin{array}{r}
\left(1+|\mathbf{q}|^{b}\right)^{\frac{1}{2}} G_{c}^{\frac{1}{2}} \frac{\tilde{\phi}^{\left(i_{k}\right)}}{M^{\frac{\beta}{2}}} \rightarrow\left(1+|\mathbf{q}|^{b}\right)^{\frac{1}{2}} G_{c}^{\frac{1}{2}} \frac{\tilde{\phi}}{M^{\frac{\beta}{2}}} \text { in } L^{2}(\Omega \times D), \\
\left(1+|\mathbf{q}|^{b}\right)^{\frac{1}{2}}|\mathbf{q}| \frac{\tilde{\phi}^{\left(i_{k}\right)}}{M^{\frac{\beta}{2}}} \rightarrow\left(1+|\mathbf{q}|^{b}\right)^{\frac{1}{2}}|\mathbf{q}| \frac{\tilde{\phi}}{M^{\frac{\beta}{2}}} \text { in } L^{2}(\Omega \times D), \\
\left(1+|\mathbf{q}|^{b}\right)^{\frac{1}{2}} M^{\frac{\beta}{2}} \nabla_{x}\left(\frac{\tilde{\phi}^{\left(i_{k}\right)}}{M^{\beta}}\right) \rightarrow\left(1+|\mathbf{q}|^{b}\right)^{\frac{1}{2}} M^{\frac{\beta}{2}} \nabla_{x}\left(\frac{\tilde{\phi}}{M^{\beta}}\right) \text { in } \mathbf{L}^{2}(\Omega \times D), \\
\left(1+|\mathbf{q}|^{b}\right)^{\frac{1}{2}} W_{c}^{\frac{1}{2}} M^{\frac{\beta}{2}} \nabla_{q}\left(\frac{\tilde{\phi}^{\left(i_{k}\right)}}{M^{\beta}}\right) \rightarrow\left(1+|\mathbf{q}|^{b}\right)^{\frac{1}{2}} W_{c}^{\frac{1}{2}} M^{\frac{\beta}{2}} \nabla_{q}\left(\frac{\tilde{\phi}}{M^{\beta}}\right) \text { in } \mathbf{L}^{2}(\Omega \times D), \\
\mathbf{C}\left(\tilde{\phi}^{\left(i_{k}\right)}\right) \rightarrow \mathbf{C}(\tilde{\phi}) \text { in } \mathbf{L}^{2}(\Omega), \\
\tilde{\mathbf{v}}^{\left(i_{k}\right)} \rightarrow \tilde{\mathbf{v}} \text { in } \mathbf{H}^{1}(\Omega), \\
\tilde{\mathbf{v}}^{\left(i_{k}\right)} \rightarrow \tilde{\mathbf{v}} \text { in } \mathbf{L}^{r}(\Omega) .
\end{array}
$$

Here, we know that $\forall \mathbf{w} \in \mathbf{V},\left|\hat{\mathbf{u}}^{n-1}\right||\mathbf{w}| \in L^{2}(\Omega)$ by the Hölder inequality and the Sobolev embedding $\mathbf{V} \hookrightarrow \mathbf{L}^{4}(\Omega)$. Further, from (A.13), we have $\forall \mathbf{w} \in \mathbf{V}$,

$$
\lim _{i_{k} \rightarrow \infty} \int_{\Omega}\left[\left(\hat{\mathbf{u}}^{n-1} \cdot \nabla_{x}\right) \tilde{\mathbf{v}}^{\left(i_{k}\right)}\right] \cdot \mathbf{w} d \mathbf{x}=\int_{\Omega}\left[\left(\hat{\mathbf{u}}^{n-1} \cdot \nabla_{x}\right) \tilde{\mathbf{v}}\right] \cdot \mathbf{w} d \mathbf{x}
$$


Hence, we can deduce from (4.17), (A.7), on noting (3.20) and (A.12)-(A.13), that $\tilde{\mathbf{v}} \in \mathbf{V}$ and $\tilde{\phi} \in Y_{b}^{\beta}$ satisfy

$$
b\left(\hat{\mathbf{u}}^{n-1}\right)(\tilde{\mathbf{v}}, \mathbf{w})=\int_{\Omega}\left[\hat{\mathbf{u}}^{n-1} \cdot \mathbf{w}-\Delta t \kappa \omega \mathbf{C}(\tilde{\phi}): \nabla_{x}\left(\mathbf{w}_{\alpha}\right)\right] d \mathbf{x} \quad \forall \mathbf{w} \in \mathbf{V} .
$$

Similarly, we can also deduce from (4.18), (A.6) and (A.8)-(A.11), on noting (4.13), (A.4)-(A.5), that $\hat{\mathbf{v}} \in \mathbf{Y}^{r}$ and $\tilde{\phi} \in Y_{b}^{\beta}$ satisfy

$$
a(\hat{\mathbf{v}})(\tilde{\phi}, \varphi)=\int_{\Omega \times D}\left[\left(1+|\mathbf{q}|^{b}\right) G_{c}+\triangle t \frac{b}{2}|\mathbf{q}|^{b} A\left(\hat{\mathbf{u}}^{n-1}\right)\right] \frac{\hat{\psi}^{n-1} \varphi}{M^{\beta}} d \mathbf{q} d \mathbf{x} \quad \forall \varphi \in Y_{b}^{\beta} .
$$

Combining the above two relations, we obtain $\tilde{\mathbf{v}}=\mathbf{G}(\hat{\mathbf{v}}) \in \mathbf{Y}^{r}$. Therefore, we have $\lim _{i \rightarrow \infty}\left\|\mathbf{G}\left(\hat{\mathbf{v}}^{(i)}\right)-\mathbf{G}(\hat{\mathbf{v}})\right\|_{L^{r}(\Omega)}=0$, which implies that $\mathbf{G}$ is continuous.

From the compactness of the embedding $\mathbf{V} \hookrightarrow \mathbf{L}^{r}(\Omega), r \in(d, 6)$, we finally verify that $\mathbf{G}$ is compact. This completes the proof.

The proof of Lemma 4.3. From the first and second terms on the left-hand side of (4.45), on noting (4.40)-(4.39), we have (4.46).

It follows from (4.46) that, $\forall \eta \in L^{2}\left(0, T ; \mathbf{C}_{0}^{\infty}(\Omega \times D)\right)$, as $\triangle t \rightarrow 0_{+}$,

$$
\int_{0}^{T} \int_{\Omega \times D}\left(1+|\mathbf{q}|^{b}\right)^{\frac{1}{2}} \mathbf{q} \frac{\hat{\psi}^{\triangle t,+}}{M^{\frac{\beta}{2}}} \cdot \eta d \mathbf{q} d \mathbf{x} d t \rightarrow \int_{0}^{T} \int_{\Omega \times D}\left(1+|\mathbf{q}|^{b}\right)^{\frac{1}{2}} \mathbf{q} \frac{\hat{\psi}}{M^{\frac{\beta}{2}}} \cdot \eta d \mathbf{q} d \mathbf{x} d t .
$$

Thus, this implies (4.47), on noting the fifth term on the left-hand side of (4.45), and the denseness of $\mathbf{C}_{0}^{\infty}(\Omega \times D)$ in $\mathbf{L}^{2}(\Omega \times D)$.

From (3.2) and (4.46), we get for any $\eta \in L^{2}\left(0, T ; \mathbf{C}_{0}^{\infty}(\Omega \times D)\right)$, as $\triangle t \rightarrow 0_{+}$,

$$
\begin{aligned}
& \int_{0}^{T} \int_{\Omega \times D}\left(1+|\mathbf{q}|^{b}\right)^{\frac{1}{2}} W_{c}^{\frac{1}{2}} M^{\frac{\beta}{2}} \nabla_{q}\left(\frac{\hat{\psi}^{\triangle t,+}}{M^{\beta}}\right) \cdot \eta d \mathbf{q} d \mathbf{x} d t \\
= & -\int_{0}^{T} \int_{\Omega \times D} \frac{\hat{\psi}^{\triangle t,+}}{M^{\beta}} \nabla_{q} \cdot\left(\left(1+|\mathbf{q}|^{b}\right)^{\frac{1}{2}} W_{c}^{\frac{1}{2}} M^{\frac{\beta}{2}} \eta\right) d \mathbf{q} d \mathbf{x} d t \\
& \rightarrow-\int_{0}^{T} \int_{\Omega \times D} \frac{\hat{\psi}}{M^{\beta}} \nabla_{q} \cdot\left(\left(1+|\mathbf{q}|^{b}\right)^{\frac{1}{2}} W_{c}^{\frac{1}{2}} M^{\frac{\beta}{2}} \eta\right) d \mathbf{q} d \mathbf{x} d t .
\end{aligned}
$$

Therefore, (4.49) follows immediately from the above relation by using the third term on the left-hand side of (4.45) and the denseness of $\mathbf{C}_{0}^{\infty}(\Omega \times D)$ in $\mathbf{L}^{2}(\Omega \times D)$. By a similar argument, we can get (4.48).

Next, we consider (4.50). From (3.4) and (4.46), we can easily obtain that

$$
\left\|\left(1+|\mathbf{q}|^{b}\right)^{\frac{1}{2}} \hat{\psi}^{\triangle t(, \pm)}\right\|_{L^{\infty}\left(0, T ; L^{2}(\Omega \times D)\right)} \leq C(\alpha, T) .
$$

Then

$$
\left(1+|\mathbf{q}|^{b}\right)^{\frac{1}{2}} \hat{\psi}^{\triangle t(, \pm)} \stackrel{*}{\rightarrow}\left(1+|\mathbf{q}|^{b}\right)^{\frac{1}{2}} \hat{\psi} \text { in } L^{\infty}\left(0, T ; L^{2}(\Omega \times D)\right) .
$$

Define a smooth function sequence $\left\{\zeta_{m}(\mathbf{q})\right\}_{m \geq 1}$ :

$$
\left\{\begin{array}{l}
\zeta_{m}(\mathbf{q})=1, \quad \text { for }|\mathbf{q}| \leq m-1 \\
\zeta_{m}(\mathbf{q})=0, \quad \text { for }|\mathbf{q}| \geq m \\
0 \leq \zeta_{m}(\mathbf{q}) \leq 1, \text { for } m-1<|\mathbf{q}|<m
\end{array}\right.
$$


Then for any $\eta(\mathbf{x}, t) \in L^{1}\left(0, T ; \mathbf{C}_{0}^{\infty}(\Omega)\right)$, we have $\zeta_{m} \eta \mathbf{q} \in L^{1}\left(0, T ; \mathbf{L}^{2}(\Omega \times D)\right)$. Furthermore, from (A.15), we obtain as $\triangle t \rightarrow 0_{+}$,

$$
\int_{0}^{T} \int_{\Omega \times D}\left(\hat{\psi}^{\triangle t(, \pm)} \mathbf{q}\right) \cdot\left(\zeta_{m} \eta \mathbf{q}\right) d \mathbf{q} d \mathbf{x} d t \rightarrow \int_{0}^{T} \int_{\Omega \times D}(\hat{\psi} \mathbf{q}) \cdot\left(\zeta_{m} \eta \mathbf{q}\right) d \mathbf{q} d \mathbf{x} d t
$$

that is,

$$
\int_{0}^{T} \int_{\Omega \times D}\left(\hat{\psi}^{\triangle t(, \pm)} \mathbf{q q}^{T}\right):\left(\zeta_{m} \eta\right) d \mathbf{q} d \mathbf{x} d t \rightarrow \int_{0}^{T} \int_{\Omega \times D}\left(\hat{\psi} \mathbf{q} \mathbf{q}^{T}\right):\left(\zeta_{m} \eta\right) d \mathbf{q} d \mathbf{x} d t
$$

Letting $m \rightarrow \infty$, we can get for any $\eta(\mathbf{x}, t) \in L^{1}\left(0, T ; \mathbf{C}_{0}^{\infty}(\Omega)\right)$,

$$
\int_{0}^{T} \int_{\Omega} \mathbf{C}\left(\hat{\psi}^{\triangle t(, \pm)}\right): \eta d \mathbf{x} d t \rightarrow \int_{0}^{T} \int_{\Omega} \mathbf{C}(\hat{\psi}): \eta d \mathbf{x} d t \text { as } \triangle t \rightarrow 0_{+} .
$$

From (4.34)-(4.35) and (A.15), we have $\mathbf{C}(\hat{\psi}) \in L^{\infty}\left(0, T ; \mathbf{L}^{2}(\Omega)\right)$. Then (4.50) follows immediately from (A.16) in virtue of the fact that $\mathbf{C}(\hat{\psi}), \mathbf{C}\left(\hat{\psi}^{\triangle t(, \pm)}\right) \in L^{\infty}\left(0, T ; \mathbf{L}^{2}(\Omega)\right)$ and the denseness of $\mathbf{C}_{0}^{\infty}(\Omega)$ in $\mathbf{L}^{2}(\Omega)$. Finally, the non-negativity of $\hat{\psi}$ follows from that of $\hat{\psi}^{\triangle t(, \pm)}$.

In the same way, (4.44) and the last term on the left-hand side of (4.45) imply (4.51)-(4.53). Using the compactness of embedding $\mathbf{V} \hookrightarrow \mathbf{L}^{r}(\Omega)$, Lemma 3.6 and (4.52)-(4.53) yields (4.54) for $\hat{\mathbf{u}}^{\triangle t}$. We now prove (4.54) for $\hat{\mathbf{u}}^{\triangle t, \pm}$. It follows from (4.41) and the second term on the left-hand side of (4.44) that

$$
\left\|\hat{\mathbf{u}}^{\triangle t}-\hat{\mathbf{u}}^{\triangle t, \pm}\right\|_{L^{2}\left(0, T ; L^{2}(\Omega)\right)}^{2} \leq C \triangle t .
$$

Moreover, the Gagliardo-Nirenberg inequality (3.30) yields that for any $\eta \in$ $L^{2}\left(0, T ; H_{0}^{1}(\Omega)\right)$,

$$
\begin{aligned}
\|\eta\|_{L^{2}\left(0, T ; L^{r}(\Omega)\right)} & \leq C\left\{\int_{0}^{T}\|\eta\|_{L^{2}(\Omega)}^{2(1-\gamma)}\left\|\nabla_{x} \eta\right\|_{L^{2}(\Omega)}^{2 \gamma} d t\right\}^{\frac{1}{2}} \\
& \leq C\|\eta\|_{L^{2}\left(0, T ; L^{2}(\Omega)\right)}^{1-\gamma}\|\eta\|_{L^{2}\left(0, T ; H^{1}(\Omega)\right)}^{\gamma},
\end{aligned}
$$

where $r \in[2, \infty)$ if $d=2$, and $r \in[2,6)$ if $d=3$ and $\gamma=d\left(\frac{1}{2}-\frac{1}{r}\right) \in[0,1)$. Thus, combining (A.17)-(A.18) and (4.54) for $\hat{\mathbf{u}}^{\triangle t}$ yields (4.54) for $\hat{\mathbf{u}}^{\triangle t, \pm}$. Finally, from (3.21), (4.51) and (4.54), we have (4.55)-(4.56). The lemma is thus proved.

Acknowledgement. The authors would like to express their gratitude to the referee for his constructive comments and suggestions.

\section{REFERENCES}

[1] J.W. Barrett, Ch. Schwab and E. Süli, Existence of global weak solutions for some polymeric flow models, Math. Models Methods Appl. Sci., 15, 939-983, 2005.

[2] J.W. Barrett and E. Süli, Existence of global weak solutions to kinetic models for dilute polymers, Multi. Model. Simul., 6, 506-546, 2007.

[3] S. Barza, J. Pečarić and L.E. Persson, Carlson type inequalities, J. Inequal. Appl., 2, 121-135, 1998.

[4] A. Bonito, P. Clément and M. Picasso, Mathematical analysis of a simplified Hookean dumbbells model arising from viscoelastic flows, J. Evol. Equ., 6, 381-398, 2006. 
[5] M. Del Pino and J. Dolbeault, The optimal euclidean $L^{p}$-Sobolev logarithmic inequality, J. Funct. Anal., 197, 151-161, 2003.

[6] M. Doi and S.F. Edwards, The Theory of Polymer Dynamics, Oxford University Press, Oxford, 1986.

[7] R.B. Bird, R.C. Armstrong and O. Hassager, Dynamics of Polymeric Liquids, Vol 1: Fluid Mechanics, Wiley Interscience, New Yourk, 1987.

[8] R.B. Bird, O. Hassager, R.C. Armstrong and C.F. Curtiss, Dynamics of Polymeric Liquids, Vol 2: Kinetic Theory, Wiley Interscience, New York, 1987.

[9] W. E, T. Li and P. Zhang, Well-posedness for the dumbell model of polymeric fluids, Commun. Math. Phys., 248, 409-427, 2004.

[10] E. Fernández-Cara, F. Guillén and R.R. Ortega, Mathematicall Modeling and Analysis of Viscoelastic Fluids of the Oldroyd Kind, Handbook of Numerical Analysis, Vol. VIII, Elsevier Science B.V, 2002.

[11] C. Foias, D.D. Holm and E.S. Titi, The Navier-Stokes- $\alpha$ model of fluid turbulence, Physica D, 152-153, 505-519, 2001.

[12] D. Gilbarg and N.S. Trudinger, Elliptic Partial Differential Equations of Second Order, Reprint of the 1998 ed., Springer-Verlag, 2001.

[13] V. Girault and P.A. Raviart, Finite Element Methods for Navier-Stokes Equations, Springer Ser. Comp. Math., 5, Springer-verlag, 1986.

[14] B. Jourdain, T. Lelievre and C. Le Bris, Numerical analysis of micro-macro simulations of polymeric fluid flows: a simple case, Math. Models Appl. Sci., 12, 1205-1243, 2002.

[15] B. Jourdain, T. Lelièvre and C. Le Bris, Existence of solution for a micro-macro model of polymeric fluid: the FENE model, J. Funct. Anal., 209, 162-193, 2004.

[16] J. Kačur, Method of Rothe in Evolution Equations, Teubner-Texte zur Mathematik, BSB B.G. Teubner Verlagsgesellschaft, Leipzig, 80, 1985.

[17] R.G. Larson, The Structure and Rheology of Complex Fluids, Oxford University Press, Oxford, 1999.

[18] T. Li, H. Zhang and P. Zhang, Local existence for the dumbell model of polymeric fluids, Commun. PDE, 29, 903-923, 2004.

[19] T. Li and P. Zhang, Mathematical analysis of multi-scale models of complex fluids, Commun. Math. Sci., 5, 1-51, 2007.

[20] F.H. Lin, C. Liu and P. Zhang, On a micro-macro model for polymeric fluids near equilibrium, Commun. Pure Appl. Math., 60, 838-866, 2006.

[21] P.L. Lions and N. Masmoudi, Global solutions for some Oldroyd models of non-Newtonian flows, Chin. Ann. Math. B, 21, 131-146, 2000.

[22] P.L. Lions and N. Masmoudi, Global existence of weak solutions to some micro-macro models, C. R. Acad. Sci. Paris, Ser. I, 345, 15-20, 2007.

[23] A. Lozinski, Spectral methods for kinetic theory models of viscoelastic fluids, Thèse $\mathrm{N}^{\circ} 2860$ (2003), École Polytechnique Fédérale de Lausanne, 2003.

[24] A. Lozinski, R.G. Owens and J. Fang, A Fokker-Planck-based numerical method for modelling nonhomogeneous flows of dilute polymeric solutions, J. Non-Newtonian Fluid Mech., 122, 273-286, 2004.

[25] H.C. Öttinger, Stochastic Processes in Polymeric Liquids, Springer-Verlag, Berlin and New York, 1996.

[26] K. Rektorys, The Method of Discretization in Time and Partial Differential Equations, Mathematics and its Applications, D. Reidel Publishing, Dordrecht, 4, 1982.

[27] M. Renardy, An existence theorem for model equations resulting from kinetic theories of polymer solutions, SIAM J. Math. Anal., 22 (1), 313-327, 199.

[28] E. Rothe, Zweidimensionale parabolische Randwertaufgaben als Grenzfall eindimensionaler Randwertaufgaben, Math. Ann., 102, 650-670, 1930.

[29] J.D. Schieber, Generalized Brownian configuration field for Fokker-Planck equations including center-of-mass diffusion, J. Non-Newtonian Fluid Mech., 135, 179-181, 2006.

[30] R. Temam, Navier-Stokes Equations. Theory and Numerical Analysis, Third Edition, NorthHolland, 1984.

[31] H. Zhang and P. Zhang, Local existence for the FENE-dumbbell model of polymeric fluids, Archive for Rational Mechanics and Analysis, 181, 373-400, 2006. 